\title{
Quantum and Classical Integrable Systems
}

\author{
M.A. Semenov-Tian-Shansky \\ 1 Physique Mathématique, Université de Bourgogne, Dijon, France \\ 2 Steklov Mathematical Institute, St.Petersburg, Russia
}

\begin{abstract}
The key concept discussed in these lectures is the relation between the Hamiltonians of a quantum integrable system and the Casimir elements in the underlying hidden symmetry algebra. (In typical applications the latter is either the universal enveloping algebra of an affine Lie algebra, or its q-deformation.) A similar relation also holds in the classical case. We discuss different guises of this very important relation and its implication for the description of the spectrum and the eigenfunctions of the quantum system. Parallels between the classical and the quantum cases are thoroughly discussed.
\end{abstract}

\section{Introduction}

The study of exactly solvable quantum mechanical models is at least as old as Quantum Mechanics itself. Over the last fifteen years there has been a major development aimed at a unified treatment of many examples known previously and, more importantly, at a systematic construction of new ones. The new method nicknamed the Quantum Inverse Scattering Method was largely created by L. D. Faddeev and his school in St. Petersburg as a quantum counterpart of the Classical Inverse Scattering Method, and has brought together many ideas believed to be unrelated. [Besides the Classical Inverse Scattering Method, one should mention the profound results of R.Baxter in Quantum Statistical Mechanics (which, in turn, go back to the work of L. Onsager, E. Lieb and many others) and the seminal paper of $\mathrm{H}$. Bethe on the ferromagnet model in which the now famous Bethe Ansatz was introduced.] It also allowed to unravel highly nontrivial algebraic structures, the Quantum Group Theory being one of its by-products.

The origins of QISM lie in the study of concrete examples; it is designed as a working machine which produces quantum systems together with their spectra, the quantum integrals of motion, and their joint eigenvectors. In the same spirit, the Classical Inverse Scattering Method (along with its ramifications) is a similar tool to produce examples of classical integrable systems together with their solutions. In these lectures it is virtually impossible to follow the historic way in which this method has been developed, starting with the famous papers of Gardner, Greene, Kruskal and Miura (1967), and Lax (1968). (A good introduction close to the ideas of QISM may be found in (Faddeev and Takhtajan (1987)).) I would like to comment only on one important turning point which gave the impetus to invent the Quantum Inverse Scattering Method. In 1979 L. D. Faddeev exposed in his seminar the draft paper of B. Kostant on the quantization of the Toda lattice (Kostant (1979)) which reduced the problem to the representation theory of semisimple Lie groups. This was an indication that classical completely integrable systems have an intimate relation to Lie groups and should also have exactly solvable quantum counterparts. In the same talk Faddeev introduced the now famous $R L_{1} L_{2}=L_{2} L_{1} R$ commutation relations 
for quantum Lax matrices which could be extracted from R. Baxter's works on quantum transfer matrices. The research program outlined in that talk and implemented over the next few years was twofold: On the one hand, the Yang-Baxter equation and the related algebra have directly led to exact solutions of several quantum models, such as the quantum sine-Gordon equation. On the other hand, connections with group theory and the orbits method resulted in a systematic treatment of numerous examples in the classical setting (Reyman and Semenov-Tian-Shansky (1994)). One major problem which has remained unsettled for more than a decade is to fill the gap between the two approaches, and in particular, to explain the group theoretical meaning of the Bethe Ansatz.

With hindsight we can now understand why this problem could not be resolved immediately. First of all, while classical integrable systems are related to ordinary Lie groups, quantum systems quite often (though not always, cf. the discussion in section 3) require the full machinery of Quantum Groups. The background took several years to prepare (Drinfeld (1987a)). Second, even the simplest systems such as the open Toda lattice require a very advanced technique of representation theory (Kostant (1979), Semenov-Tian-Shansky (1994a)). The Toda lattice is peculiar, since the underlying 'hidden symmetry' group is finite-dimensional. All main examples are related to (classical or quantum) affine Lie algebras. The representation theory of affine algebras which is instrumental in the study of integrable systems has been developed only in recent years (semi-infinite cohomologies, Wakimoto modules, critical level representations, cf. Feigin and Frenkel (1992), Feigin, Frenkel and Reshetikhin (1994), Frenkel (1995)).

The present lectures do not give a systematic overview of the Quantum Inverse Scattering Method (several good expositions are available, cf. Bogolyubov et al. (1994), Faddeev (1980, 1984, 1995), Faddeev and Takhtajan (1979), Kulish and Sklyanin (1981), Sklyanin (1992)). Instead, I shall try to explain the parallels between quantum and classical systems and the 'correspondence principles' which relate the quantum and the classical case.

As already mentioned, the first key observation is that integrable systems always have an ample hidden symmetry. (Fixing this symmetry provides some rough classification of the associated examples. A nontrivial class of examples is related to loop algebras or, more generally, to their q-deformations. This is the class of examples I shall consider below.) Two other key points are the role of (classical or quantum) R-matrices and of the Casimir elements which give rise to the integrals of motion.

This picture appears in several different guises depending on the type of examples in question. The simplest (so-called linear) case corresponds to classical systems which are modelled on coadjoint orbits of Lie algebras; a slightly more complicated group of examples is classical systems modelled on Poisson Lie groups or their Poisson submanifolds. In the quantum setting the difference between these two cases is deeper: while in the former case the hidden symmetry algebra remains the same, quantization of the latter leads to Quantum Groups. Still, for loop algebras the quantum counterpart of the main construction is nontrivial even in the linear case; the point is that the universal enveloping algebra of a loop algebra has a trivial center which reappears only after central extension at the critical value of the central charge. Thus to tackle the quantum case one needs the full machinery of representation theory of loop algebras. (By contrast, in the classical case one mainly deals with the evaluation representations which allow to reduce the solution of the equations of motion to a problem in algebraic geometry.) 
The study of integrable models may be divided into two different parts. The first one is, so to say, kinematic: it consists in the choice of appropriate models together with their phase spaces or the algebra of observables and of their Hamiltonians. The second one is dynamical; it consists, classically, in the description of solutions or of the action-angle variables. The quantum counterpart consists in the description of the spectra, the eigenvectors, and of the various correlation functions. The algebraic scheme proved to be very efficient in the description of kinematics. (Quantum Group Theory may be regarded as a by-product of this kinematic problem.) The description of spectra at the present stage of the theory remains model dependent. The standard tool for constructing the eigenvectors of the quantum Hamiltonians which was the starting point of QISM is the algebraic Bethe Ansatz. Until very recently, its interpretation in terms of the representation theory was lacking. This problem has been finally settled by Feigin, Frenkel and Reshetikhin (1994) for an important particular model with linear commutation relations (the Gaudin model); remarkably, their results follow the general pattern outlined above. A similar treatment of quantum models related to q-deformed affine algebras is also possible, although the results in this case are still incomplete. One should be warned that much of the 'experimental material' on Quantum Integrability still resists general explanations. I would like to mention in this respect the deep results of E.K.Sklyanin (Sklyanin (1992), Sklyanin (1995)) relating the Bethe Ansatz to the separation of variables; see also (Harnad and Winternitz (1993), Kuznetsov (1994)).

Acknowledgements. The present lectures were prepared for the CIMPA Winter School on Nonlinear Systems which was held in Pondicherry, India, in January 1996. I am deeply grateful to the Organizing Committee of the School and to the staff of the Pondicherry University for their kind hospitality. I would also like to thank Prof. Y. Kosmann-Schwarzbach for her remarks on the draft text of these lectures.

\section{Generalities}

A quantum mechanical system is a triple consisting of an associative algebra with involution $\mathcal{A}$ called the algebra of observables, an irreducible $\star$-representation $\pi$ of $\mathcal{A}$ in a Hilbert space $\mathfrak{H}$ and a distinguished self-adjoint observable $\mathcal{H}$ called the Hamiltonian. A typical question to study is the description of the spectrum and the eigenvectors of $\pi(\mathcal{H})$. Quantum mechanical systems usually appear with their classical counterparts. By definition, a classical mechanical system is again specified by its algebra of observables $\mathcal{A}_{c l}$ which is a commutative associative algebra equipped with a Poisson bracket (i.e., a Lie bracket which also satisfies the Leibniz rule

$$
\{a, b c\}=\{a, b\} c+\{a, c\} b
$$

in other words, a Poisson bracket is a derivation of $\mathcal{A}_{c l}$ with respect to both its arguments), and a Hamiltonian $\mathcal{H} \in \mathcal{A}_{c l}$. A commutative algebra equipped with a Poisson bracket satisfying the Leibniz rule is called a Poisson algebra. Speaking informally, a quantum algebra of observables $\mathcal{A}$ arises as a deformation of the commutative algebra $\mathcal{A}_{c l}$ determined by the Poisson bracket. 
In these lectures we shall not be concerned with the quantization problem in its full generality (cf. Bayen, Flato et al. (1977), Weinstein (1994)). However, it will always be instructive to compare quantum systems with their classical counterparts.

The algebraic language which starts with Poisson algebras makes the gap between classical and quantum mechanics as narrow as possible; in practice, however, we also need the dual language based on the notion of the phase space. Roughly speaking, the phase space is the spectrum of the Poisson algebra. Accurate definition depends on the choice of a topology in the Poisson algebra. We shall not attempt to discuss these subtleties and shall always assume that the underlying phase space is a smooth manifold and that the Poisson algebra is realized as the algebra of functions on this manifold. In the examples we have in mind, Poisson algebras always have an explicit geometric realization of this type. The Poisson bracket itself is then as usual represented by a bivector field on the phase space satisfying certain differential constraints which account for the Jacobi identity. This gives the definition of a Poisson manifold which is dual to the notion of a Poisson algebra. Geometry of Poisson manifolds has numerous obvious parallels with the representation theory. Recall that the algebraic version of the representation theory is based on the study of appropriate ideals in an associative algebra. For a Poisson algebra we have a natural notion of a Poisson ideal (i.e., a subalgebra which is an ideal with respect to both structures); the dual notion is that of a Poisson submanifold of a Poisson manifold. The classical counterpart of Hilbert space representations of an associative algebra is the restriction of functions to various Poisson submanifolds. Poisson submanifolds are partially ordered by inclusion; minimal Poisson submanifolds are those for which the induced Poisson structure is nondegenerate. (This means that the center of the Lie algebra of functions contains only constants.) Minimal Poisson submanifolds always carry a symplectic structure and form a stratification of the Poisson manifold; they are called symplectic leaves. Restriction of functions to symplectic leaves gives a classical counterpart of the irreducible representations of associative algebras.

Let $\mathcal{M}$ be a Poisson manifold, $\mathcal{H} \in C^{\infty}(\mathcal{M})$. A classical system $(\mathcal{M}, \mathcal{H})$ is called integrable if the commutant of the Hamiltonian $\mathcal{H}$ in $\mathcal{A}_{c l}$ contains an abelian algebra of the maximal possible rank. (A technical definition is provided by the well known Liouville theorem.)

Let us recall that the key idea which has started the modern age in the study of classical integrable systems is to bring them into Lax form. In the simplest case, the definition of a Lax representation may be given as follows. Let $(\mathcal{A}, \mathcal{M}, \mathcal{H})$ be a classical mechanical system. Let $\mathcal{F}_{t}: \mathcal{M} \rightarrow \mathcal{M}$ be the associated flow on $\mathcal{M}$ (defined at least locally). Suppose that $\mathfrak{g}$ is a Lie algebra. A mapping $L: \mathcal{M} \rightarrow \mathfrak{g}$ is called a Lax representation of $(\mathcal{A}, \mathcal{M}, \mathcal{H})$ if the following conditions are satisfied:

(i) The flow $\mathcal{F}_{t}$ factorizes over $\mathfrak{g}$, i.e., there exists a (local) flow $F_{t}: \mathfrak{g} \rightarrow \mathfrak{g}$ such that the following diagram is commutative.

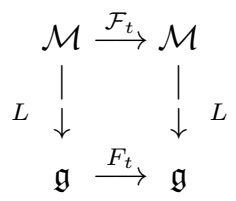

(ii) The quotient flow $F_{t}$ on $\mathfrak{g}$ is isospectral, i.e., it is tangent to adjoint orbits in $\mathfrak{g}$. 
Clearly, $L(x) \in \mathfrak{g}$ for any $x \in \mathcal{M}$; hence we may regard $L$ as a 'matrix with coefficients in $\mathcal{A}=C^{\infty}(\mathcal{M})^{\prime}$, i.e., as an element of $\mathfrak{g} \otimes \mathcal{A}$; the Poisson bracket on $\mathcal{A}$ extends to $\mathfrak{g} \otimes \mathcal{A}$ by linearity. Property (ii) means that there exists an element $M \in \mathfrak{g} \otimes \mathcal{A}$ such that $\{\mathcal{H}, L\}=$ $[L, M]$.

Let $(\rho, V)$ be a (finite-dimensional) linear representation of $\mathfrak{g}$. Then $L_{V}=\rho \otimes i d(L) \in$ $E n d V \otimes \mathcal{A}$ is a matrix valued function on $\mathcal{M}$; the coefficients of its characteristic polynomial $P(\lambda)=\operatorname{det}\left(L_{V}-\lambda\right)$ are integrals of the motion.

One may replace in the above definition a Lie algebra $\mathfrak{g}$ with a Lie group $G$; in that case isospectrality means that the flow preserves conjugacy classes in $G$. In a more general way, the Lax operator may be a difference or a differential operator. The difference case is of particular importance, since the quantization of difference Lax equations is the core of QISM and a natural source of Quantum Groups. (Cf. section 3 below)

There is no general way to find a Lax representation for a given system (even if it is known to be completely integrable). However, there is a systematic way to produce examples of such representations. An ample source of such examples is provided by the general construction described in the next section.

\subsection{Basic Theorem: Linear Case}

The basic construction outlined in this section (summarized in Theorem 2.3 below) goes back to Kostant (1979) and Adler (1979) in some crucial cases; its relation with the r-matrix method was established in (Semenov-Tian-Shansky (1983)). We shall state it using the language of symmetric algebras which simplifies its generalization to the quantum case. Let $\mathfrak{g}$ be a Lie algebra over $k$ (where $k=\mathbb{R}$ or $\mathbb{C}$ ). Let $\mathcal{S}(\mathfrak{g}$ ) be the symmetric algebra of $\mathfrak{g}$. Recall that there is a unique Poisson bracket on $\mathcal{S}(\mathfrak{g})$ (called the Lie-Poisson bracket) which extends the Lie bracket on $\mathfrak{g}$ (see, for example, (Cartier (1994)). This Poisson bracket is more frequently discussed from the 'spectral' point of view. Namely, let $\mathfrak{g}^{*}$ be the linear dual of $\mathfrak{g}$; the natural pairing $\mathfrak{g} \times \mathfrak{g}^{*} \rightarrow k$ extends to the 'evaluation map' $\mathcal{S}(\mathfrak{g}) \times \mathfrak{g}^{*} \rightarrow k$ which induces a canonical isomorphism of $\mathcal{S}(\mathfrak{g})$ with the space of polynomials $P\left(\mathfrak{g}^{*}\right)$; thus $\mathfrak{g}^{*}$ is a linear Poisson manifold and $\mathcal{S}(\mathfrak{g}) \simeq P\left(\mathfrak{g}^{*}\right)$ is the corresponding algebra of observables. Linear functions on $\mathfrak{g}^{*}$ form a subspace in $P\left(\mathfrak{g}^{*}\right)$ which may be identified with $\mathfrak{g}$. The Lie-Poisson bracket on $\mathfrak{g}^{*}$ is uniquely characterized by the following properties:

1. The Poisson bracket of linear functions on $\mathfrak{g}^{*}$ is again a linear function.

2. The restriction of the Poisson bracket to $\mathfrak{g} \subset P\left(\mathfrak{g}^{*}\right)$ coincides with the Lie bracket in $\mathfrak{g}$.

Besides the Lie-Poisson structure on $\mathcal{S}(\mathfrak{g})$ we shall need its Hopf structure. Further on we shall deal with other more complicated examples of Hopf algebras, so it is probably worth recalling the general definitions (though we shall not use them in full generality until section 4). Recall that a Hopf algebra is a set $(A, m, \Delta, \epsilon, S)$ consisting of an associative algebra $A$ over $k$ with multiplication $m: A \otimes A \rightarrow A$ and unit element 1 , the coproduct $\Delta: A \rightarrow A \otimes A$, the counit $\epsilon: A \rightarrow k$ and the antipode $S: A \rightarrow A$ which satisfy the following axioms 
- $m: A \otimes A \rightarrow A, \Delta: A \rightarrow A \otimes A, i: k \rightarrow A: \alpha \longmapsto \alpha \cdot 1, \epsilon: A \rightarrow k$ are homomorphisms of algebras.

- The following diagrams are commutative:

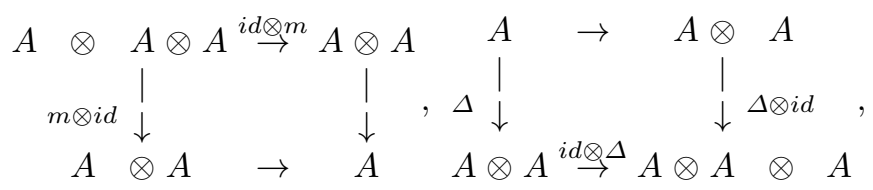

(these diagrams express associativity of the product $m$ and the coassociativity of the coproduct $\Delta$, respectively),
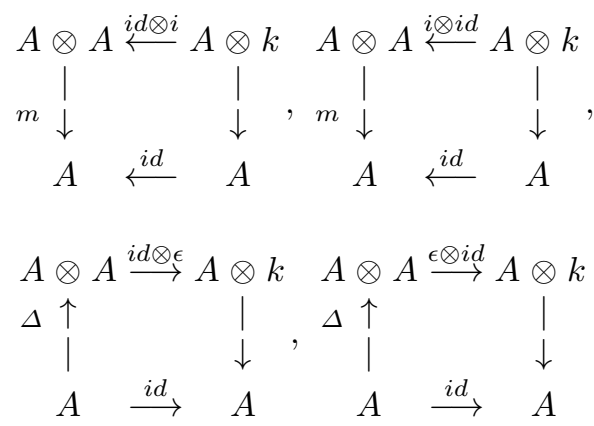

(these diagrams express, respectively, the properties of the unit element $1 \in A$ and of the counit $\left.\epsilon \in A^{*}\right)$.

- The antipode $S$ is an antihomomorphism of algebras and the following diagrams are commutative:

$$
\begin{aligned}
& A \stackrel{\Delta}{\longrightarrow} A \otimes A \stackrel{i d \otimes S}{\longrightarrow} A \otimes A \stackrel{m}{\longrightarrow} A \\
& \searrow^{\epsilon} \quad k^{i \nearrow} \\
& A \stackrel{\Delta}{\longrightarrow} A \otimes A \stackrel{S \otimes i d}{\longrightarrow} A \otimes A \stackrel{m}{\longrightarrow} A \\
& \searrow^{\epsilon} \quad \nearrow_{i}
\end{aligned}
$$

If $(A, m, 1, \Delta, \epsilon, S)$ is a Hopf algebra, its linear dual $A^{*}$ is also a Hopf algebra; moreover, the coupling $A \otimes A^{*} \rightarrow k$ interchanges the roles of product and coproduct. Thus we have

$$
\langle m(a \otimes b), \varphi\rangle=\langle a \otimes b, \Delta \varphi\rangle .
$$


The Hopf structure on the symmetric algebra $\mathcal{S}(\mathfrak{g})$ is determined by the structure of the additive group on $\mathfrak{g}^{*}$. Namely, let $\mathcal{S}(\mathfrak{g}) \times \mathfrak{g}^{*} \rightarrow k:(a, X) \longmapsto a(X)$ be the evaluation map; since $\mathcal{S}(\mathfrak{g}) \otimes \mathcal{S}(\mathfrak{g}) \simeq \mathcal{S}(\mathfrak{g} \oplus \mathfrak{g}) \simeq P\left(\mathfrak{g}^{*} \oplus \mathfrak{g}^{*}\right)$, this map extends to $\mathcal{S}(\mathfrak{g}) \otimes \mathcal{S}(\mathfrak{g})$. We have

$$
\Delta a(X, Y)=a(X+Y), \epsilon(a)=a(0), S(a)(X)=a(-X)
$$

(we shall sometimes write $S(a)=a^{\prime}$ for brevity).

For future reference we recall also that the coproducts in the universal enveloping algebra $\mathcal{U}(\mathfrak{g})$ and in $\mathcal{S}(\mathfrak{g})$ coincide (in other words, $\mathcal{U}(\mathfrak{g})$ is canonically isomorphic to $\mathcal{S}(\mathfrak{g})$ as a coalgebra).

Let us now return to the discussion of the Poisson structure on $\mathcal{S}(\mathfrak{g})$, i.e., of the Lie-Poisson bracket of the Lie algebra $\mathfrak{g}$. It is well known that the Lie-Poisson bracket is always degenerate; its symplectic leaves are precisely the coadjoint orbits of the corresponding Lie group.

Proposition 2.1 The center of $\mathcal{S}(\mathfrak{g})$ (regarded as a Poisson algebra) coincides with the subalgebra $I=\mathcal{S}(\mathfrak{g})^{\mathfrak{g}}$ of adg-invariants in $\mathcal{S}(\mathfrak{g})$ (called Casimir elements).

Restrictions of Casimir elements to symplectic leaves are constants; so fixing the values of Casimirs provides a rough classification of symplectic leaves, although it is not true in general that different symplectic leaves are separated by the Casimirs. (In the semisimple case there are enough Casimirs to separate generic orbits.)

Fix an element $\mathcal{H} \in \mathcal{S}(\mathfrak{g})$; it defines a derivation $D_{\mathcal{H}}$ of $\mathcal{S}(\mathfrak{g})$,

$$
D_{\mathcal{H}} \varphi=\{\mathcal{H}, \varphi\} .
$$

By duality, this derivation determines a (local) Hamiltonian flow on $\mathfrak{g}^{*}$. Since the Lie-Poisson bracket on $\mathfrak{g}^{*}$ is degenerate, this flow splits into a family of independent flows which are confined to Poisson submanifolds in $\mathfrak{g}^{*}$. Thus a Hamiltonian $\mathcal{H}$ defines Hamiltonian flows on the coadjoint orbits in $\mathfrak{g}^{*}$. In the special case when $\mathcal{H} \in I$ all these flows are trivial. However, one may still use Casimir elements to produce nontrivial equations of motion if the Poisson structure on $\mathcal{S}(\mathfrak{g})$ is properly modified, and this is the way in which Lax equations associated with $\mathfrak{g}$ do arise. The formal definition is as follows.

Let $r \in E n d \mathfrak{g}$ be a linear operator; we shall say that $r$ is a classical $r$-matrix if the $r$-bracket

$$
[X, Y]_{r}=\frac{1}{2}([r X, Y]+[X, r Y]), X, Y \in \mathfrak{g},
$$

satisfies the Jacobi identity. In that case we get two different Lie brackets on the same underlying linear space $\mathfrak{g}$. We shall assume that $r$ satisfies the following stronger condition called the modified classical Yang-Baxter identity:

$$
[r X, r Y]-r([r X, Y]+[X, r Y])=-[X, Y], X, Y \in \mathfrak{g} .
$$

Proposition 2.2 Identity (2.8) implies the Jacobi identity for (2.7). 
Remark 2.1 Identity (2.8) is of course only a sufficient condition; however, we prefer to impose it from the very beginning, since it assures a very important factorization property (see below).

Let $(\mathfrak{g}, r)$ be a Lie algebra equipped with a classical r-matrix $r \in E n d \mathfrak{g}$ satisfying (2.8). Let $\mathfrak{g}_{r}$ be the corresponding Lie algebra (with the same underlying linear space). Put $r_{ \pm}=\frac{1}{2}(r \pm i d$ ); then (2.8) implies that $r_{ \pm}: \mathfrak{g}_{r} \rightarrow \mathfrak{g}$ are Lie algebra homomorphisms. Let us extend them to Poisson algebra morphisms $\mathcal{S}\left(\mathfrak{g}_{r}\right) \rightarrow \mathcal{S}(\mathfrak{g})$ which we shall denote by the same letters. These morphisms also agree with the standard Hopf structure on $\mathcal{S}(\mathfrak{g}), \mathcal{S}\left(\mathfrak{g}_{r}\right)$. Define the action

$$
\mathcal{S}\left(\mathfrak{g}_{r}\right) \otimes \mathcal{S}(\mathfrak{g}) \rightarrow \mathcal{S}(\mathfrak{g})
$$

by setting

$$
x \cdot y=\sum r_{+}\left(x_{i}^{(1)}\right) y r_{-}\left(x_{i}^{(2)}\right)^{\prime}, x \in \mathcal{S}\left(\mathfrak{g}_{r}\right), y \in \mathcal{S}(\mathfrak{g}),
$$

where $\Delta x=\sum x_{i}^{(1)} \otimes x_{i}^{(2)}$ is the coproduct and $a \mapsto a^{\prime}$ is the antipode map.

Theorem 2.3 (i) $\mathcal{S}(\mathfrak{g})$ is a free graded $\mathcal{S}\left(\mathfrak{g}_{r}\right)$-module generated by $1 \in \mathcal{S}(\mathfrak{g})$. (ii) Let $i_{r}: \mathcal{S}(\mathfrak{g})$ $\rightarrow \mathcal{S}\left(\mathfrak{g}_{r}\right)$ be the induced isomorphism of graded linear spaces; its restriction to $I=\mathcal{S}(\mathfrak{g})^{\mathfrak{g}}$ is a morphism of Poisson algebras. (iii) Assume, moreover, that $\mathfrak{g}$ is equipped with a nondegenerate invariant bilinear form; the induced mapping $\mathfrak{g}_{r}^{*} \rightarrow \mathfrak{g}$ defines a Lax representation for all Hamiltonians $\mathcal{H}=i_{r}(\widehat{\mathcal{H}}), \widehat{\mathcal{H}} \in \mathcal{S}(\mathfrak{g})^{\mathfrak{g}}$.

The geometric meaning of theorem 2.3 is very simple. In fact, it becomes more transparent if one uses the language of Poisson manifolds rather than the dual language of Poisson algebras. (However, it is this more cumbersome language that may be generalized to the quantum case.) Since $\mathfrak{g} \simeq \mathfrak{g}_{r}$ as linear spaces, the polynomial algebras $P\left(\mathfrak{g}^{*}\right)$ and $P\left(\mathfrak{g}_{r}^{*}\right)$ are also isomorphic as graded linear spaces (though not as Poisson algebras). The subalgebra $I \subset P\left(\mathfrak{g}^{*}\right)$ remains commutative with respect to the Lie-Poisson bracket of $\mathfrak{g}_{r}$; moreover, the flows in $\mathfrak{g}^{*}$ which correspond to the Hamiltonians $\mathcal{H} \in I$ are tangent to two systems of coadjoint orbits in $\mathfrak{g}^{*}$, the orbits of $\mathfrak{g}$ and of $\mathfrak{g}_{r}$. The latter property is trivial, since all Hamiltonian flows in $\mathfrak{g}_{r}^{*}$ are tangent to coadjoint orbits; the former is an immediate corollary of the fact that $I=\mathcal{S}(\mathfrak{g})^{\mathfrak{g}} \simeq P\left(\mathfrak{g}^{*}\right)^{\mathfrak{g}}$ is the subalgebra of coadjoint invariants. (As we mentioned, in the semisimple case generic orbits are level surfaces of the Casimirs.) Finally, if $\mathfrak{g}$ carries an invariant inner product, the coadjoint and the adjoint representations of $\mathfrak{g}$ are equivalent and $I$ consists precisely of spectral invariants.

Let $\mathfrak{u} \subset \mathfrak{g}_{r}$ be an ideal, $\mathfrak{s}=\mathfrak{g}_{r} / \mathfrak{u}$ the quotient algebra, $p: \mathcal{S}\left(\mathfrak{g}_{r}\right) \rightarrow \mathcal{S}(\mathfrak{s})$ the canonical projection; restricting $p$ to the subalgebra $I_{r}=i_{r}\left(\mathcal{S}(\mathfrak{g})^{\mathfrak{g}}\right)$ we get a Poisson commutative subalgebra in $\mathcal{S}(\mathfrak{s})$; we shall say that the corresponding elements of $\mathcal{S}(\mathfrak{s})$ are obtained by specialization.

The most common examples of classical r-matrices are associated with decompositions of Lie algebras. Let $\mathfrak{g}$ be a Lie algebra with a nondegenerate invariant inner product, $\mathfrak{g}_{+}, \mathfrak{g}_{-} \subset \mathfrak{g}$ its two Lie subalgebras such that $\mathfrak{g}=\mathfrak{g}_{+} \dot{+} \mathfrak{g}_{-}$as a linear space. Let $P_{+}, P_{-}$be the projection operators associated with this decomposition; the operator

$$
r=P_{+}-P_{-}
$$


satisfies (2.8); moreover, the Lie algebra $\mathfrak{g}_{r}$ splits into two parts, $\mathfrak{g}_{r} \simeq \mathfrak{g}_{+} \oplus \mathfrak{g}_{-}$. Let us choose $\mathcal{A}=\mathcal{S}\left(\mathfrak{g}_{+}\right)$as our algebra of observables. Let $\mathfrak{g}_{+}^{\perp}, \mathfrak{g}_{-}^{\perp}$ be the orthogonal complements of $\mathfrak{g}_{+}, \mathfrak{g}_{-}$ in $\mathfrak{g}$. Then $\mathfrak{g}=\mathfrak{g}_{+}^{\perp} \oplus \mathfrak{g}_{-}^{\perp}$ and we may identify $\mathfrak{g}_{+}^{*} \simeq \mathfrak{g}_{-}^{\perp}, \mathfrak{g}_{-}^{*} \simeq \mathfrak{g}_{+}^{\perp}$. Let $L \in \mathfrak{g}_{-}^{\perp} \otimes \mathfrak{g}_{+} \subset \mathfrak{g}_{-}^{\perp} \otimes \mathcal{S}\left(\mathfrak{g}_{+}\right)$ be the canonical element (that is, $L=\sum e_{i} \otimes e^{i}$, where $\left\{e^{i}\right\}$ is a basis in $\mathfrak{g}_{+}$and $\left\{e_{i}\right\}$ the dual basis in $\mathfrak{g}_{+}^{*} \simeq \mathfrak{g}_{-}^{\perp}$; using the canonical isomorphism $\mathfrak{g}_{-}^{\perp} \otimes \mathfrak{g}_{+} \simeq \operatorname{Hom}\left(\mathfrak{g}_{+}^{*}, \mathfrak{g}_{-}^{\perp}\right)$, we may regard $L$ as an embedding $\mathfrak{g}_{+}^{*} \hookrightarrow \mathfrak{g}_{-}^{\perp}$. Let $P: \mathcal{S}(\mathfrak{g}) \rightarrow \mathcal{S}\left(\mathfrak{g}_{-}\right)$be the projection onto $\mathcal{S}\left(\mathfrak{g}_{-}\right)$in the decomposition

$$
\mathcal{S}(\mathfrak{g})=\mathcal{S}\left(\mathfrak{g}_{-}\right) \oplus \mathfrak{g}_{+} \mathcal{S}(\mathfrak{g}) .
$$

Corollary 2.4 (i) The restriction of $P$ to the subalgebra $I=\mathcal{S}(\mathfrak{g})^{\mathfrak{g}}$ of Casimir elements is a Poisson algebra homomorphism. (ii) L defines a Lax representation for all Hamiltonian equations of motion defined by the Hamiltonians $\overline{\mathcal{H}}=P(\mathcal{H}), \mathcal{H} \in I$. (iii) The corresponding Hamiltonian flows on $\mathfrak{g}_{+}^{*} \simeq \mathfrak{g}_{-}^{\perp} \subset \mathfrak{g}$ preserve intersections of coadjoint orbits of $\mathfrak{g}_{+}$with the adjoint orbits of $\mathfrak{g}$.

One sometimes calls $L$ the universal Lax operator; restricting the mapping $L: \mathfrak{g}_{+}^{*} \hookrightarrow \mathfrak{g}_{-}^{\perp}$ to various Poisson submanifolds in $\mathfrak{g}_{+}^{*}$ we get Lax representations for particular systems.

The situation is especially simple when $\mathfrak{g}_{+}, \mathfrak{g}_{-} \subset \mathfrak{g}$ are isotropic subspaces with respect to the inner product in $\mathfrak{g}$; then $\mathfrak{g}_{+}=\mathfrak{g}_{+}^{\perp}, \mathfrak{g}_{-}=\mathfrak{g}_{-}^{\perp}, \mathfrak{g}_{+} \simeq \mathfrak{g}_{-}^{*}$. In that case $\left(\mathfrak{g}_{+}, \mathfrak{g}_{-}, \mathfrak{g}\right)$ is referred to as a Manin triple. This case is of particular importance, since then both $\mathfrak{g}$ and its Lie subalgebras $\mathfrak{g}_{+}, \mathfrak{g}_{-}$carry an additional structure of Lie bialgebra.

Definition 2.1 Let $\mathfrak{a}$ be a Lie algebra, $\mathfrak{a}^{*}$ its dual; assume that $\mathfrak{a}^{*}$ is equipped with a Lie bracket $[,]_{*}: \mathfrak{a}^{*} \wedge \mathfrak{a}^{*} \rightarrow \mathfrak{a}^{*}$. The brackets on $\mathfrak{a}$ and $\mathfrak{a}^{*}$ are said to be compatible if the dual map $\delta: \mathfrak{a} \rightarrow \mathfrak{a} \wedge \mathfrak{a}$ is a 1-cocycle on $\mathfrak{a}$ (with values in the wedge square of the adjoint module). A pair $\left(\mathfrak{a}, \mathfrak{a}^{*}\right)$ with consistent Lie brackets is called a Lie bialgebra.

One can prove that this definition is actually symmetric with respect to $\mathfrak{a}, \mathfrak{a}^{*} ;$ in other words, if $\left(\mathfrak{a}, \mathfrak{a}^{*}\right)$ is a Lie bialgebra, so is $\left(\mathfrak{a}^{*}, \mathfrak{a}\right)$.

Proposition 2.5 Let $\left(\mathfrak{g}_{+}, \mathfrak{g}_{-}, \mathfrak{g}\right)$ be a Manin triple. Identify $\mathfrak{g}^{*}$ with $\mathfrak{g}$ by means of the inner product and equip it with the $r$-bracket associated with $r=P_{+}-P_{-}$; then (i) $\left(\mathfrak{g}, \mathfrak{g}^{*}\right)$ is a Lie bialgebra. (ii) $\left(\mathfrak{g}_{+}, \mathfrak{g}_{-}\right)$is a Lie sub-bialgebra of $\left(\mathfrak{g}, \mathfrak{g}^{*}\right)$. (iii) Conversely, if $\left(\mathfrak{a}, \mathfrak{a}^{*}\right)$ is a Lie bialgebra there exists a unique Lie algebra $\mathfrak{d}=d\left(\mathfrak{a}, \mathfrak{a}^{*}\right)$ called the double of $\left(\mathfrak{a}, \mathfrak{a}^{*}\right)$ such that

$-\mathfrak{d}=\mathfrak{a} \dot{+} \mathfrak{a}^{*}$ as a linear space,

$-\mathfrak{a}, \mathfrak{a}^{*} \subset \mathfrak{d}$ are Lie subalgebras,

- The canonical bilinear form on $\mathfrak{d}$ induced by the natural pairing between $\mathfrak{a}$ and $\mathfrak{a}^{*}$ is add -invariant.

In a more general way, let $r \in E n d \mathfrak{g}$ be a classical r-matrix on a Lie algebra $\mathfrak{g}$ which is equipped with a fixed inner product. Identify $\mathfrak{g}_{r}$ with the dual of $\mathfrak{g}$ by means of the inner product. Assume that $r$ is skew and satisfies the modified Yang-Baxter identity. Then $\left(\mathfrak{g}, \mathfrak{g}^{*}\right)$ is a Lie bialgebra; it is usually called a factorizable Lie bialgebra. Let us once more list the properties of factorizable Lie bialgebras for future reference: 
- $\mathfrak{g}$ is equipped with a nondegenerate inner product.

- $\mathfrak{g}^{*}$ is identified with $\mathfrak{g}$ as a linear space by means of the inner product; the Lie bracket in $\mathfrak{g}^{*}$ is given by

$$
[X, Y]_{*}=\frac{1}{2}([r X, Y]+[X, r Y]), X, Y \in \mathfrak{g},
$$

$-r_{ \pm}=\frac{1}{2}(r \pm i d)$ are Lie algebra homomorphisms $\mathfrak{g}^{*} \rightarrow \mathfrak{g} ;$ moreover, $r_{+}^{*}=-r_{-}$, and $r_{+}-$ $r_{-}=i d$.

Lie bialgebras are particularly important, since the associated Lie-Poisson structures may be extended to Lie groups (cf. section 3).

2.1.1. Hamiltonian Reduction. Theorem 2.3 admits a very useful global version which survives quantization. To formulate this theorem we shall first recall some basic facts about Hamiltonian reduction.

Let $G$ be a Lie group with Lie algebra $\mathfrak{g}$. Let $T^{*} G$ be the cotangent bundle of $G$ equipped with the canonical symplectic structure. Let $B \subset G$ be a Lie subgroup with Lie algebra $\mathfrak{b}$. The action of $B$ on $G$ by right translations extends canonically to a Hamiltonian action $B \times T^{*} G$ $\rightarrow T^{*} G$. This means that the vector fields on $T^{*} G$ generated by the corresponding infinitesimal action of $\mathfrak{b}$ are globally Hamiltonian; moreover, the mapping

$$
\mathfrak{b} \rightarrow C^{\infty}\left(T^{*} G\right): X \longmapsto h_{X}
$$

which assigns to each $X \in \mathfrak{b}$ the Hamiltonian of the corresponding vector field is a homomorphism of Lie algebras. In particular, this map is linear and hence for each $X \in \mathfrak{g}, x \in T^{*} G$

$$
h_{X}(x)=\langle X, \mu(x)\rangle,
$$

where $\mu: T^{*} G \rightarrow \mathfrak{b}^{*}$ is the so-called moment mapping. Choose a trivialization $T^{*} G \simeq G \times \mathfrak{g}^{*}$ by means of left translations; then the action of $B$ is given by

$$
b:(g, \xi) \longmapsto\left(g b^{-1},\left(A d^{*} b\right)^{-1} \xi\right) .
$$

The corresponding moment map $\mu: T^{*} G \rightarrow \mathfrak{b}^{*}$ is

$$
\mu:(g, \xi) \longmapsto-\left.\xi\right|_{\mathfrak{b}},
$$

where $\left.\xi\right|_{\mathfrak{b}}$ means the restriction of $\xi \in \mathfrak{g}^{*}$ to $\mathfrak{b} \subset \mathfrak{g}$. The moment mapping is equivariant; in other words, the following diagram is commutative

$$
\begin{aligned}
& B \times T^{*} G \rightarrow T^{*} G
\end{aligned}
$$

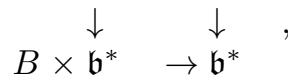

where $B \times \mathfrak{b}^{*} \rightarrow \mathfrak{b}^{*}$ is the ordinary coadjoint action.

Recall that the reduction procedure consists of two steps: 
- Fix the value of the moment map $\mu$ and consider the level surface

$$
\mathcal{M}_{F}=\left\{x \in T^{*} G ; \mu(x)=F\right\}, F \in \mathfrak{b}^{*}
$$

Speaking in physical terms, we impose on $T^{*} G$ linear constraints which fix the moment $\mu: T^{*} G \rightarrow \mathfrak{b}^{*}$.

- Take the quotient of $\mathcal{M}_{F}$ over the action of the stabilizer of $F$ in $B$.

(Due to the equivariance of $\mu$, the stabilizer $B^{F} \subset B$ preserves $\mathcal{M}_{F}$.)

The resulting manifold $\overline{\mathcal{M}}_{F}$ is symplectic; it is called the reduced phase space obtained by reduction over $F \in \mathfrak{b}^{*}$. A particularly simple case is the reduction over $0 \in \mathfrak{b}^{*}$; in that case the stabilizer coincides with $B$ itself. We shall denote the reduced space $\mathcal{M}_{0} / B$ by $T^{*} G / / B$.

In a slightly more general fashion, we may start with an arbitrary Hamiltonian $B$-space $\mathcal{M}$ and perform the reduction over the zero value of the moment map getting the reduced space $\mathcal{M} / / B$.

Let $\mathrm{S}_{G}$ be the category of Hamiltonian $G$-spaces; recall that (up to a covering) homogeneous Hamiltonian $G$-spaces are precisely the coadjoint orbits of $G$. We shall define a functor (called symplectic induction, cf. Kazhdan, Kostant and Sternberg (1978)),

$$
\operatorname{Ind}_{B}^{G}: \mathrm{S}_{B} \rightsquigarrow \mathrm{S}_{G},
$$

which associates to each Hamiltonian $B$-space a Hamiltonian $G$-space. Namely, we put

$$
\operatorname{Ind}_{B}^{G}(\mathcal{M})=T^{*} G \times \mathcal{M} / / B
$$

where the manifold $T^{*} G \times \mathcal{M}$ is equipped with the symplectic structure which is the difference of symplectic forms on $T^{*} G$ and on $\mathcal{M}$ and $B$ acts on $T^{*} G \times \mathcal{M}$ diagonally (hence the moment map on $T^{*} G \times \mathcal{M}$ is the difference of the moment maps on $T^{*} G$ and on $\mathcal{M}$ ). The structure of the Hamiltonian $G$-space is induced on $T^{*} G \times \mathcal{M} / / B$ by the action of $G$ on $T^{*} G$ by left translations.

The most simple case of symplectic induction is when the coadjoint orbit consists of a single point. In representation theory, this corresponds to representations induced by 1-dimensional representations of a subgroup. Let now $F \in \mathfrak{b}^{*}$ be an arbitrary element; let $\mathcal{O}_{F}$ be its coadjoint orbit, $\mathcal{K}_{F}=\operatorname{Ind}_{B}^{G}\left(\mathcal{O}_{F}\right)$. It is sometimes helpful to have a different construction of $\mathcal{K}_{F}$ (in much the same way as in representation theory it is helpful to have different realizations of a given representation). In many case it is possible to induce $\mathcal{K}_{F}$ starting from a smaller subgroup $L \subset B$ and its single-point orbit (a similar trick in representation theory means that we wish to induce a given representation from a 1-dimensional one). The proper choice of $L$ is the Lagrangian subgroup $L_{F} \subset B$ subordinate to $F$ (whenever it exists). Recall that a Lie subalgebra $\mathfrak{l}_{F} \subset \mathfrak{b}$ is called a Lagrangian subalgebra subordinate to $F \in \mathfrak{b}^{*}$ if $a d^{*} \mathfrak{l}_{F} \cdot F \subset T_{F} \mathcal{O}_{F}$ is a Lagrangian subspace of the tangent space to the coadjoint orbit of $F$ (with respect to the Kirillov form on $\left.T_{F} \mathcal{O}_{F}\right)$. This condition implies that $\left.F\right|_{\left[\mathfrak{l}_{F}, \mathfrak{l}_{F}\right]}=0$; in other words, $F$ defines a character of $\mathfrak{l}_{F}$. This is of course tantamount to saying that $F$ is a single-point orbit of $\mathfrak{l}_{F}$. To define the corresponding Lagrangian subgroup $L_{F} \subset B$ let us observe that $\mathfrak{l}_{F}$ always coincides with its own normalizer in $\mathfrak{b}$. Let us define $L_{F} \subset B$ as the normalizer of $\mathfrak{l}_{F}$ in $B$ (this definition fixes the group of components of $L_{F}$ which may be not necessarily connected). 
Proposition 2.6 Let $L_{F} \subset B$ be a Lagrangian subgroup subordinate to $F$; then $T^{*} G \times \mathcal{O}_{F} / / B \simeq$ $T^{*} G \times\{F\} / / L_{F}$.

In the next section we shall discuss an example of a coadjoint orbit with real polarization.

Let us now return to the setting of theorem 2.3. Consider the action $G \times G \times G \rightarrow G$ by left and right translations:

$$
\left(h, h^{\prime}\right): x \longmapsto h x h^{-1} ;
$$

this action may be lifted to the Hamiltonian action $G \times G \times T^{*} G \rightarrow T^{*} G$. Let us choose again a trivialization $T^{*} G \simeq G \times \mathfrak{g}^{*}$ by means of left translations; then

$$
\left(h, h^{\prime}\right):(g, \xi) \longmapsto\left(h g h^{\prime-1},\left(A d^{*} h^{\prime}\right)^{-1} \xi\right) .
$$

The corresponding moment map $\mu: T^{*} G \rightarrow \mathfrak{g}^{*} \oplus \mathfrak{g}^{*}$ is

$$
\mu:(g, \xi) \longmapsto\left(\xi,-\left(A d^{*} g\right)^{-1} \xi\right) .
$$

Using the left trivialization of $T^{*} G$ we may extend polynomial functions $\varphi \in P\left(\mathfrak{g}^{*}\right) \simeq \mathcal{S}(\mathfrak{g})$ to left-invariant functions on $T^{*} G$ which are polynomial on the fiber. The Casimir elements $\mathcal{H} \in \mathcal{S}(\mathfrak{g})^{\mathfrak{g}}$ give rise to bi-invariant functions; thus the corresponding Hamiltonian systems admit reduction with respect to any subgroup $S \subset G \times G$.

Lemma 2.7 Let $S \subset G \times G$ be a Lie subgroup, $\mathfrak{s} \subset \mathfrak{g} \oplus \mathfrak{g}$ its Lie subalgebra, $p: \mathfrak{g}^{*} \oplus \mathfrak{g}^{*} \rightarrow \mathfrak{s}^{*}$ the canonical projection, $S \times T^{*} G \rightarrow T^{*} G$ the restriction to $H$ of the action (2.12). This action is Hamiltonian and the corresponding moment map is $\mu_{S}=p \circ \mu$.

Now let us assume again that $(\mathfrak{g}, r)$ is a Lie algebra equipped with a classical r-matrix $r \in$ End $\mathfrak{g}$ satisfying (2.8). Combining the Lie algebra homomorphisms $r_{ \pm}: \mathfrak{g}_{r} \rightarrow \mathfrak{g}$ we get an embedding $i_{r}: \mathfrak{g}_{r} \rightarrow \mathfrak{g} \oplus \mathfrak{g}$; we may identify the Lie group which corresponds to $\mathfrak{g}_{r}$ with the subgroup $G_{r} \subset G \times G$ which corresponds to the Lie subalgebra $\mathfrak{g}_{r} \subset \mathfrak{g} \oplus \mathfrak{g}$. Fix $F \in \mathfrak{g}_{r}^{*}$ and let $\mathcal{O}_{F} \subset \mathfrak{g}_{r}^{*}$ be its coadjoint orbit. We want to apply to $\mathcal{O}_{F}$ the 'symplectic induction' procedure. Since half of $G_{r}$ is acting by left translations and the other half by right translations, the action of $G$ on the reduced space will be destroyed. However, the Casimir elements survive reduction.

Theorem 2.8 (i) The reduced symplectic manifold $T^{*} G \times \mathcal{O}_{F} / / G_{r}$ is canonically isomorphic to $\mathcal{O}_{F}$. (ii) Extend Casimir elements $\mathcal{H} \in \mathcal{S}(\mathfrak{g})^{\mathfrak{g}} \simeq P\left(\mathfrak{g}^{*}\right)$ to $G \times G$-invariant functions on $T^{*} G$; these functions admit reduction with respect to $G_{r}$; the reduced Hamiltonians coincide with those described in theorem 2.3, namely, $\mathcal{H}_{\text {red }}=\left.i_{r}(\mathcal{H})\right|_{\mathcal{O}_{F}}$; the quotient Hamiltonian flow on $\mathcal{O}_{F}$ which corresponds to $\mathcal{H} \in \mathcal{S}(\mathfrak{g})^{\mathfrak{g}}$ is described by the Lax equation.

Theorem 2.8 allows to get explicit formulae for the solutions of Lax equations in terms of the factorization problem in $G$. In these lectures we are mainly interested in its role for quantization.

The construction of Lax equations based on theorem 2.3 may be applied to finite-dimensional semisimple Lie algebras (Kostant (1979)). However, its really important applications are connected with loop algebras, which possess sufficiently many Casimirs. We shall describe two 
examples both of which have interesting quantum counterparts: the open Toda lattice (this is the example first treated in (Kostant (1979))) and the so-called generalized Gaudin model (its quantum counterpart was originally proposed in (Gaudin (1983))).

\subsection{Two Examples}

2.2.1. Generalized Toda Lattice. The Toda Hamiltonian describes transverse oscillations of a 1-dimensional cyclic molecule with the nearest-neighbour interaction around the equilibrium configuration. Thus the phase space is $\mathcal{M}=\mathbb{R}^{2 n}$ with the canonical Poisson bracket; the Hamiltonian is

$$
\mathcal{H}=\frac{1}{2} \sum_{i=1}^{n} p_{i}^{2}+\sum_{i=1}^{n-1} \exp \left(q_{i}-q_{i+1}\right)+\exp \left(q_{n}-q_{1}\right) .
$$

Removing the last term in the potential energy gives the open Toda lattice whose behaviour is qualitatively different (all potentials are repulsive, and the Hamiltonian describes the scattering of particles in the conical valley

$$
C_{+}=\left\{q=\left(q_{1}, \ldots, q_{n}\right) \in \mathbb{R}^{n} ; q_{i}-q_{i+1} \leq 0, i=1, \ldots, n-1\right\}
$$

with steep walls). The generalized open Toda lattice may be associated to an arbitrary semisimple Lie algebra. (Generalized periodic Toda lattices correspond to affine Lie algebras.) I shall briefly recall the corresponding construction (cf. Kostant (1979), Goodman and Wallach (1982), Reyman and Semenov-Tian-Shansky (1994)).

Let $\mathfrak{g}$ be a real split semisimple Lie algebra, $\sigma: \mathfrak{g} \rightarrow \mathfrak{g}$ a Cartan involution, $\mathfrak{g}=\mathfrak{k} \oplus \mathfrak{p}$ the corresponding Cartan decomposition (i.e., $\sigma=i d$ on $\mathfrak{k}, \sigma=-i d$ on $\mathfrak{p}$ ). Fix a split Cartan subalgebra $\mathfrak{a} \subset \mathfrak{p} ;$ let $\Delta \subset \mathfrak{a}^{*}$ be the root system of $(\mathfrak{g}, \mathfrak{a})$. For $\alpha \in \Delta$ let

$$
\mathfrak{g}_{\alpha}=\{X \in \mathfrak{g} ; a d H \cdot X=\alpha(H) X, H \in \mathfrak{a}\}
$$

be the corresponding root space. Fix an order in the system of roots and let $\Delta_{+} \subset \Delta$ be the set of positive roots, $P \subset \Delta_{+}$the corresponding set of simple roots. Put $\mathfrak{n}=\oplus_{\alpha \in \Delta_{+}} \mathfrak{g}_{\alpha}$. Let $\mathfrak{b}=\mathfrak{a}+\mathfrak{n}$; recall that $\mathfrak{b}$ is a maximal solvable subalgebra in $\mathfrak{g}$ (Borel subalgebra). We have

$$
\mathfrak{g}=\mathfrak{k} \dot{+} \mathfrak{a} \dot{+} \mathfrak{n}
$$

(the Iwasawa decomposition). Equip $\mathfrak{g}$ with the standard inner product (the Killing form). Clearly, we have $\mathfrak{k}^{\perp}=\mathfrak{p}, \mathfrak{b}^{\perp}=\mathfrak{n}$; thus the dual of $\mathfrak{b}$ is modelled on $\mathfrak{p}$, and we may regard $\mathfrak{p}$ as a $\mathfrak{b}$-module with respect to the coadjoint representation. Let $G$ be a connected split semisimple Lie group with finite center which corresponds to $\mathfrak{g}, G=A N K$ its Iwasawa decomposition which corresponds to the decomposition (2.15) of its Lie algebra.

Remark 2.2 The Iwasawa decomposition usually does not give rise to a Lie bialgebra structure on $\mathfrak{g}$; indeed, the subalgebras $\mathfrak{k}$ and $\mathfrak{b}$ are not isotropic (unless $\mathfrak{g}$ is a complex Lie algebra considered as an algebra over $\mathbb{R}$ ). In other words, the classical r-matrix associated with the decomposition (2.15) is not skew. We shall briefly recall the construction of the so-called standard skew-symmetric classical r-matrix on a semisimple Lie algebra in Section 2.2.3 below. 
The Borel subalgebra admits a decreasing filtration $\mathfrak{b} \supset \mathfrak{b}^{(1)} \supset \mathfrak{b}^{(2)} \supset \ldots$, where $\mathfrak{b}^{(1)}=$ $[\mathfrak{b}, \mathfrak{b}]=\mathfrak{n}, \mathfrak{b}^{(2)}=[\mathfrak{n}, \mathfrak{n}], \ldots, \mathfrak{b}^{(k)}=\left[\mathfrak{n}, \mathfrak{b}^{(k-1)}\right]$. By duality, there is an increasing filtration of $\mathfrak{p} \simeq \mathfrak{b}^{*}$ by $a d^{*} \mathfrak{b}$-invariant subspaces. To describe it let us introduce some notation. For $\alpha \in \Delta$ let

$$
\alpha=\sum_{\alpha_{i} \in P} m_{i} \alpha_{i}
$$

be its decomposition with respect to the simple roots; put $d(\alpha)=\sum_{\alpha_{i} \in P} m_{i}$. Put

$$
\mathfrak{d}_{p}=\bigoplus_{\{\alpha \in \Delta ; d(\alpha)=p\}} \mathfrak{g}_{\alpha}, p \neq 0, \mathfrak{d}_{0}=\mathfrak{a}, \mathfrak{n}^{(k)}=\bigoplus_{p \geq k} \mathfrak{d}_{p}
$$

Define the mapping $s: \mathfrak{g} \rightarrow \mathfrak{p}: X \longmapsto \frac{1}{2}(i d-\sigma) X$, and let $\mathfrak{p}_{k}=\oplus_{0 \leq p \leq k} s\left(\mathfrak{d}_{p}\right), k=0,1, \ldots$ It is easy to see that $\mathfrak{a}=\mathfrak{p}_{0} \subset \mathfrak{p}_{1} \subset \ldots$ are invariant with respect to the coadjoint action of $\mathfrak{b}$; moreover, $\mathfrak{p}_{k}$ is the annihilator of $\mathfrak{b}^{(k+1)}$ in $\mathfrak{p}$. Thus $\mathfrak{p}_{k}$ is set in duality with $\mathfrak{b} / \mathfrak{b}^{(k+1)}$. Put $\mathfrak{s}=\mathfrak{b} / \mathfrak{b}^{(2)}$ and let $L \in \mathfrak{p}_{1} \otimes \mathfrak{s} \subset \mathfrak{p}_{1} \otimes \mathcal{S}(\mathfrak{s})$ be the corresponding canonical element. Let $I_{\mathfrak{s}}$ be the specialization to $\mathfrak{s}$ of the subalgebra $I=\mathcal{S}(\mathfrak{g})^{\mathfrak{g}}$ of Casimir elements. An example of a Hamiltonian $H \in I_{\mathfrak{s}}$ is constructed from the Killing form:

$$
H=\frac{1}{2}(L, L),
$$

in obvious notation. To describe all others we may use the following trick. Fix a faithful representation $(\rho, V)$ of $\mathfrak{g}$ and put $L_{V}=(\rho \otimes i d) L .(\rho, V)$ is usually called the auxiliary linear representation.

Proposition 2.9 (i) $I_{\mathfrak{s}}$ is generated by $H_{k}=\operatorname{tr}_{V} L_{V}^{k} \in \mathcal{S}(\mathfrak{s}), k=1,2, \ldots$ (ii) L defines a Lax representation for any Hamiltonian equation on $\mathcal{S}(\mathfrak{s})$ with the Hamiltonian $H \in I_{\mathfrak{s}}$.

We have $\mathfrak{s}=\mathfrak{a} \ltimes \mathfrak{u}$, where $\mathfrak{u}=\mathfrak{n} /[\mathfrak{n}, \mathfrak{n}]$; the corresponding Lie group is $S=A \ltimes U, U=N / N^{\prime}$. Choose $e_{\alpha} \in \mathfrak{g}_{\alpha}, \alpha \in P$, in such a way that $\left(e_{\alpha}, e_{-\alpha}\right)=1$, and put

$$
\mathcal{O}_{T}=\left\{p+\sum_{\alpha \in P} b_{\alpha}\left(e_{\alpha}+e_{-\alpha}\right) ; p \in \mathfrak{a}, b_{\alpha}>0\right\}
$$

by construction, $\mathcal{O}_{T} \subset \mathfrak{p}_{1}$. It is convenient to introduce the parametrization $b_{\alpha}=\exp \alpha(q)$, $q \in \mathfrak{a}$.

Proposition 2.10 (i) $\mathcal{O}_{T}$ is an open coadjoint orbit of $S$ in $\mathfrak{p}_{1} \simeq \mathfrak{s}^{*}$ (ii) Restriction to $\mathcal{O}_{T}$ of the Hamiltonian (2.18) is the generalized Toda Hamiltonian

$$
H_{T}=\frac{1}{2}(p, p)+\sum_{\alpha \in P} \exp 2 \alpha(q) .
$$


The Toda orbit has the natural structure of a polarized symplectic manifold, that is, $\mathcal{O}_{T}$ admits an $S$-invariant fibering whose fibers are Lagrangian submanifolds; the description of this Lagrangian structure is a standard part of the geometric quantization program (Kirillov (1976)). More precisely, put $f=\sum_{\alpha \in P}\left(e_{\alpha}+e_{-\alpha}\right)$; let us choose $f$ as a marked point on $\mathcal{O}_{T}$.

Proposition 2.11 (i) We have $S / U \simeq A$; the isomorphism $\mathcal{O}_{T}=S \cdot f$ induces a Lagrangian fibering $\mathcal{O}_{T} \rightarrow A$ with fiber $U$. (ii) $\mathcal{O}_{T}$ is isomorphic to $T^{*} A$ as a polarized symplectic manifold. (iii) The Lagrangian subalgebra $\mathfrak{l}_{f} \subset \mathfrak{s}$ subordinate to $f$ is $\mathfrak{l}_{f}=\mathfrak{u}$.

Remark 2.3 We may of course regard $\mathcal{O}_{T}$ as a coadjoint orbit of the group $B=A N$ itself; indeed, there is an obvious projection $B \rightarrow S$ and its kernel lies in the stabilizer of $f$. The 'big' Lagrangian subgroup $\hat{L}_{f} \subset B$ subordinate to $f$ coincides with $N$.

An alternative description of the open Toda lattice which provides more detailed information on its behavior and survives quantization is based on Hamiltonian reduction. The following result is a version of theorem 2.8. Let us consider again the Hamiltonian action of $G \times G$ on $T^{*} G$. To get the generalized Toda lattice we shall restrict this action to the subgroup $G_{r}=K \times B \subset G \times G$. We may regard $\mathcal{O}_{T}$ as a $G_{r}$-orbit, the action of $K$ being trivial.

Theorem 2.12 (i) $T^{*} G \times \mathcal{O}_{T} / / G_{r}$ is isomorphic to $\mathcal{O}_{T}$ as a symplectic manifold. (ii) Let $\mathcal{H}_{2}$ be the quadratic Casimir element which corresponds to the Killing form on $\mathfrak{g}$. The Toda flow is the reduction of the Hamiltonian flow on $T^{*} G$ generated by $\mathcal{H}_{2}$.

Theorem 2.12 leads to explicit formulae for the trajectories of the Toda lattice in terms of the Iwasawa decomposition of matrices (Olshanetsky and Perelomov (1994), Goodman and Wallach (1982), Reyman and Semenov-Tian-Shansky (1994)).

Since $\mathcal{O}_{T}$ admits a real polarization, we may state the following version of proposition 2.6. Observe that if we regard $\mathcal{O}_{T}$ as a $G_{r}$-orbit, the Lagrangian subgroup subordinate to $f \in \mathcal{O}_{T}$ is $K \times N$.

Theorem 2.13 (i) $T^{*} G \times\{f\} / / K \times N$ is isomorphic to $\mathcal{O}_{T}$ as a symplectic manifold. (ii) The standard Lagrangian polarization on $T^{*} G$ by the fibers of projection $T^{*} G \rightarrow G$ gives rise to the Lagrangian polarization on $\mathcal{O}_{T}$ described in proposition 2.11.

It is easy to see that the Toda flow is again reproduced as the reduction of the Hamiltonian flow on $T^{*} G$ generated by the quadratic Hamiltonian $\mathcal{H}_{2}$.

As discussed in Section 4.1.2, theorem 2.13 gives a very simple hint to the solution of the quantization problem.

2.2.2. More examples: standard r-matrices on semisimple Lie algebras and on loop algebras. As already noted, the classical r-matrix associated with the Iwasawa decomposition of a simple Lie algebra $\mathfrak{g}$ is not skew and hence does not give rise to a Lie bialgebra structure on $\mathfrak{g}$. While this is not a disadvantage for the study of the Toda lattice, the bialgebra structure is 
of course basic in the study of the q-deformed case. Let us briefly recall the so-called standard Lie bialgebra structure on $\mathfrak{g}$. We shall explicitly describe the corresponding Manin triple.

Let $\mathfrak{g}$ be a complex simple Lie algebra, $\mathfrak{a} \subset \mathfrak{g}$ its Cartan subalgebra, $\Delta \subset \mathfrak{a}^{*}$ the root system of $(\mathfrak{g}, \mathfrak{a}), \mathfrak{b}_{+}=\mathfrak{a}+\mathfrak{n}_{+}$a positive Borel subalgebra which corresponds to some choice of order in $\Delta, \mathfrak{b}_{-}$the opposite Borel subalgebra. For $\alpha \in \Delta$ let $e_{\alpha}$ be the corresponding root space vector normalized in such a way that $\left(e_{\alpha}, e_{-\alpha}\right)=1$. We may identify $\mathfrak{a}$ with the quotient algebra $\mathfrak{b}_{ \pm} / \mathfrak{n}_{ \pm} ;$let $\pi_{ \pm}: \mathfrak{b}_{ \pm} \rightarrow \mathfrak{a}$ be the corresponding canonical projection. Put $\mathfrak{d}=\mathfrak{g} \oplus \mathfrak{g}$ (direct sum of two copies); we equip $\mathfrak{d}$ with the inner product which is the difference of the Killing forms on the first and the second copy. Let $\mathfrak{g}^{\delta} \subset \mathfrak{d}$ be the diagonal subalgebra,

$$
\mathfrak{g}^{*}=\left\{\left(X_{+}, X_{-}\right) \in \mathfrak{b}_{+} \oplus \mathfrak{b}_{-} ; \pi_{+}\left(X_{+}\right)=-\pi_{-}\left(X_{-}\right)\right\} .
$$

(Mind the important minus sign in this definition!)

Proposition 2.14 (i) $\left(\mathfrak{d}, \mathfrak{g}^{\delta}, \mathfrak{g}^{*}\right)$ is a Manin triple. (ii) The corresponding Lie bialgebra structure on $\mathfrak{g}$ is associated with the classical r-matrix

$$
r=\sum_{\alpha \in \Delta_{+}} e_{\alpha} \wedge e_{-\alpha}
$$

(iii) The Lie bialgebra $\left(\mathfrak{g}^{\delta}, \mathfrak{g}^{*}\right)$ is factorizable.

The construction described above admits a straightforward generalization for loop algebras. The associated r-matrix is called trigonometric. For future reference we shall recall its definition as well.

Let $\mathfrak{g}$ be a complex semisimple Lie algebra, let $L \mathfrak{g}=\mathfrak{g} \otimes \mathbb{C}\left[z, z^{-1}\right]$ be the loop algebra of $\mathfrak{g}$ consisting of rational functions with values in $\mathfrak{g}$ which are regular on $\mathbb{C} P(1) \backslash\{0, \infty\}$. Let $L \mathfrak{g}_{0}, L \mathfrak{g}_{\infty}$ be its local completions at 0 and $\infty$; by definition, $L \mathfrak{g}_{0}, L \mathfrak{g}_{\infty}$ consist of formal Laurent series in local parameters $z, z^{-1}$, respectively. Put $\widehat{\mathfrak{d}}=L \mathfrak{g}_{0} \oplus L \mathfrak{g}_{\infty}$ (direct sum of Lie algebras). Clearly, the diagonal embedding $L \mathfrak{g} \hookrightarrow \widehat{\mathfrak{d}}: X \longmapsto(X, X)$ is a homomorphism of Lie algebras. Let

$$
\begin{gathered}
L \mathfrak{g}_{ \pm}=\mathfrak{g} \otimes \mathbb{C}\left[\left[z^{ \pm 1}\right]\right], \\
\widehat{\mathfrak{b}}_{+}=\left\{X \in L \mathfrak{g}_{+} ; X(0) \in \mathfrak{b}_{+}\right\}, \\
\widehat{\mathfrak{b}}_{-}=\left\{X \in L \mathfrak{g}_{-} ; X(\infty) \in \mathfrak{b}_{-}\right\}
\end{gathered}
$$

(where $X(0), X(\infty)$ denote the constant term of the formal series). Combining the 'evaluation at 0 ' (respectively, at $\infty$ ) with the projections $\pi_{ \pm}: \mathfrak{b}_{ \pm} \rightarrow \mathfrak{a}$ we get two canonical projection maps $\widehat{\pi}_{ \pm}: \widehat{\mathfrak{b}}_{ \pm} \rightarrow \mathfrak{a}$. Put

$$
(L \mathfrak{g})^{*}=\left\{\left(X_{+}, X_{-}\right) \in \widehat{\mathfrak{b}}_{+} \oplus \widehat{\mathfrak{b}}_{-} ; \widehat{\pi}_{+}\left(X_{+}\right)=-\widehat{\pi}_{-}\left(X_{-}\right)\right\} \subset \widehat{\mathfrak{d}} .
$$

Let us set $(L \mathfrak{g})^{*}$ and $L \mathfrak{g}$ in duality by means of the bilinear form on $\widehat{\mathfrak{d}}$

$$
\begin{aligned}
& \left\langle\left(X_{+}, X_{-}\right),\left(Y_{+}, Y_{-}\right)\right\rangle= \\
& \quad \operatorname{Res}_{z=0}\left(X_{+}(z), Y_{+}(z)\right) d z / z+\operatorname{Res}_{z=\infty}\left(X_{-}(z), Y_{-}(z)\right) d z / z .
\end{aligned}
$$


Proposition $2.15\left(\widehat{\mathfrak{d}}, L \mathfrak{g},(L \mathfrak{g})^{*}\right)$ is a Manin triple.

Remark 2.4 Properly speaking, with our choice of a completion for $(L \mathfrak{g})^{*}$ the Lie bialgebra $\left(L \mathfrak{g},(L \mathfrak{g})^{*}\right)$ is not factorizable; indeed, our definition requires that the Lie algebra and its dual should be isomorphic as linear spaces. However, a slightly weaker assertion still holds true: the dual Lie algebra $(L \mathfrak{g})^{*}$ contains an open dense subalgebra $(L \mathfrak{g})^{\circ}=$ $\left\{\left(X_{+}, X_{-}\right) \in(L \mathfrak{g})^{*} ; X_{ \pm} \in L \mathfrak{g}\right\}$ such that the mappings $(L \mathfrak{g})^{\circ} \rightarrow L \mathfrak{g}:\left(X_{+}, X_{-}\right) \longmapsto X_{ \pm}$are Lie algebra homomorphisms and $(L \mathfrak{g})^{\circ}$ is isomorphic to L $\mathfrak{g}$ as a linear space. This subtlety is quite typical of infinite-dimensional Lie algebras.

Let us finally say a few words on the trigonometric r-matrix itself. Define the cobracket $\delta: L \mathfrak{g} \rightarrow L \mathfrak{g} \otimes L \mathfrak{g}$ by

$$
\langle\delta X, Y \otimes Z\rangle=\left\langle X,[Y, Z]_{L \mathfrak{g}^{*}}\right\rangle, X \in L \mathfrak{g}, Y, Z \in(L \mathfrak{g})^{*}
$$

i.e. as the dual of the Lie bracket in $(L \mathfrak{g})^{*}$ with respect to the pairing $(2.21)$. The element $\delta(X) \in L \mathfrak{g} \otimes L \mathfrak{g}$ may be regarded as a Laurent polynomial in two variables with values in $\mathfrak{g}$; the cobracket $(2.22)$ is then given by

$$
\delta(X)(z, w)=\left[r_{\text {trig }}\left(\frac{z}{w}\right), X_{1}(z)+X_{2}(w),\right]
$$

where $r_{\text {trig }}(z / w)$ is a rational function in $z / w$ with values in $\mathfrak{g} \otimes \mathfrak{g}$. (We use the dummy indices $1,2, \ldots$ to denote different copies of linear spaces; in other words,

$$
X_{1}(z):=X(z) \otimes 1, X_{2}(w):=1 \otimes X(w) ;
$$

below we shall frequently use this abridged tensor notation.) The explicit expression for $r_{\text {trig }}$ is given by

$$
r_{\text {trig }}(x)=t \cdot \frac{x+1}{x-1}+r,
$$

where $t \in \mathfrak{g} \otimes \mathfrak{g}$ is the canonical element (tensor Casimir) which represents the inner product $\langle$,$\rangle in \mathfrak{g}$ and $r \in \mathfrak{g} \wedge \mathfrak{g}$ is the standard classical r-matrix in $\mathfrak{g}$ given by (2.20).

Remark 2.5 Note that the expression for $r_{\text {trig }}$ which may be found in Belavin and Drinfeld (1983) is different from (2.23), since these authors use a different grading in the loop algebra (the so-called principal grading, as compared to the standard grading which we use in these lectures.) 
2.2.3. Generalized Gaudin Model. We shall return to the bialgebras described in the previous section when we shall discuss the q-deformed algebras and the quadratic Poisson Lie groups. In order to deal with our next example of an integrable system we shall use another important Lie bialgebra associated with the Lie algebra of rational functions. The example to be discussed is a model of spin-spin interaction (the so-called Gaudin model) which may be deduced as a limiting case of lattice spin models (such as the Heisenberg XXX model) for small values of the coupling constant (Gaudin (1983)). The original Gaudin model is related to the $\mathfrak{s l}(2)$ or $\mathfrak{s u}(2)$ algebras; below we shall discuss its generalization to arbitrary simple Lie algebras. The generalized Gaudin Hamiltonians also arise naturally in the semiclassical approximation to the Knizhnik-Zamolodchikov equations (Reshetikhin and Varchenko (1993)). The underlying r-matrix associated with the Gaudin model is the so called rational r-matrix which is described below.

Let $\mathfrak{g}$ be a complex simple Lie algebra. Fix a finite set $D=\left\{z_{1}, \ldots, z_{N}\right\} \subset \mathbb{C} \subset \mathbb{C} P_{1}$ and let $\mathfrak{g}(D)$ be the algebra of rational functions on $\mathbb{C} P_{1}$ with values in $\mathfrak{g}$ which vanish at infinity and are regular outside $D$. Let $\mathfrak{g}_{z_{i}}=\mathfrak{g} \otimes \mathbb{C}\left(\left(z-z_{i}\right)\right)$ be the localization of $\mathfrak{g}(D)$ at $z_{i} \in D$. [As usual, we denote by $\mathbb{C}((z))$ the algebra of formal Laurent series in $z$, and by $\mathbb{C}[[z]]$ its subalgebra consisting of formal Taylor series.] Put $\mathfrak{g}_{D}=\oplus_{z_{i} \in D} \mathfrak{g}_{z_{i}}$. There is a natural embedding $\mathfrak{g}(D) \hookrightarrow \mathfrak{g}_{D}$ which assigns to a rational function the set of its Laurent expansions at each point $z_{i} \in D$. Put $\mathfrak{g}_{z_{i}}^{+}=\mathfrak{g} \otimes \mathbb{C}\left[\left[z-z_{i}\right]\right], \mathfrak{g}_{D}^{+}=\oplus_{z_{i} \in D} \mathfrak{g}_{z_{i}}$. Then

$$
\mathfrak{g}_{D}=\mathfrak{g}_{D}^{+} \dot{+} \mathfrak{g}(D)
$$

as a linear space. [This assertion has a very simple meaning. Fix an element $X=\left(X_{i}\right)_{z_{i} \in D} \in \mathfrak{g}_{D}$; truncating the formal series $X_{i}$, we get a finite set of Laurent polynomials which may be regarded as principal parts of a rational function. Let $X^{0}$ be the rational function defined by these principal parts; it is unique up to a normalization constant which may be fixed by a condition at infinity. Expand $X^{0}$ in its Laurent series at $z=z_{i}$; by construction, $X_{i}-X_{i}^{0} \in \mathfrak{g}_{z_{i}}^{+}$ ; thus $P: X \longmapsto X_{0}$ is a projection operator from $\mathfrak{g}_{D}$ onto $\mathfrak{g}(D)$ parallel to $\mathfrak{g}_{D}^{+}$. In other words, the direct sum decomposition (2.24) is equivalent to the existence of a rational function with prescribed principal parts; this is the assertion of the well known Mittag-Leffler theorem in complex analysis. ]

Fix an inner product on $\mathfrak{g}$ and extend it to $\mathfrak{g}_{D}$ by setting

$$
\langle X, Y\rangle=\sum_{z_{i} \in D} \operatorname{Res}\left(X_{i}, Y_{i}\right) d z, X=\left(X_{i}\right)_{z_{i} \in D}, Y=\left(Y_{i}\right)_{z_{i} \in D} .
$$

(One may notice that the key difference from the formula (2.21) in the previous section is in the choice of the differential $d z$ instead of $d z / z$.) Both subspaces $\mathfrak{g}(D), \mathfrak{g}_{D}^{+}$are isotropic with respect to the inner product $(2.25)$ which sets them into duality. Thus $\left(\mathfrak{g}_{D}, \mathfrak{g}_{D}^{+}, \mathfrak{g}(D)\right)$ is a Manin triple. The linear space $\mathfrak{g}(D)$ may be regarded as a $\mathfrak{g}_{D}^{+}$-module with respect to the coadjoint representation. The action of $\mathfrak{g}_{D}^{+}$preserves the natural $\mathbb{Z}^{N}$-filtration of $\mathfrak{g}(D)$ by the order of poles. In particular, rational functions with simple poles form an invariant subspace $\mathfrak{g}(D)_{1} \subset \mathfrak{g}(D)$. Let $\mathfrak{g}_{z_{i}}^{++} \subset \mathfrak{g}_{z_{i}}^{+}$be the subalgebra consisting of formal series without constant terms. Put $\mathfrak{g}_{D}^{++}=\oplus_{z_{i} \in D} \mathfrak{g}_{z_{i}}^{++}$; clearly, $\mathfrak{g}_{D}^{++}$is an ideal in $\mathfrak{g}_{D}^{+}$and its action in $\mathfrak{g}(D)_{1}$ is trivial. 
The quotient algebra $\mathfrak{g}_{D}^{+} / \mathfrak{g}_{D}^{++}$is isomorphic to $\mathfrak{g}^{N}=\oplus_{z_{i} \in D} \mathfrak{g}$. The inner product (2.25) sets the linear spaces $\mathfrak{g}(D)_{1}$ and $\mathfrak{g}^{N}$ in duality. Let $L(z) \in \mathfrak{g}(D)_{1} \otimes \mathfrak{g}^{N}$ be the canonical element; we shall regard $L(z)$ as a matrix-valued rational function with coefficients in $\mathfrak{g}^{N} \subset S\left(\mathfrak{g}^{N}\right)$. Fix a faithful linear representation $(\rho, V)$ of $\mathfrak{g}$ (the auxiliary linear representation); it extends canonically to a representation $(\rho, V(z))$ of the Lie algebra $\mathfrak{g}(z)=\mathfrak{g} \otimes \mathbb{C}(z)$ in the space $V(z)=V \otimes \mathbb{C}(z)$. (In section 2.2.1 we have already used this auxiliary linear representation for a similar purpose, $\mathrm{cf}$. proposition 2.9 .) Put

$$
L_{V}(z)=(\rho \otimes i d) L(z)
$$

The matrix coefficients of $L_{V}(z)$ generate the algebra of observables $\mathcal{S}\left(\mathfrak{g}^{N}\right)$. The Poisson bracket relations in this algebra have a nice expression in 'tensor form', the brackets of the matrix coefficients of $L_{V} \quad$ forming a matrix in $E n d V \otimes E n d V$ with coefficients in $\mathbb{C}(u, v) \otimes \mathcal{S}\left(\mathfrak{g}^{N}\right)$; the corresponding formula, suggested for the first time by Sklyanin (1979), was the starting point of the whole theory of classical r-matrices. To describe it let us first introduce the rational $r$-matrix,

$$
r_{V}(u, v)=\frac{t_{V}}{u-v}
$$

Here $t$ is the tensor Casimir of $\mathfrak{g}$ which corresponds to the inner product in $\mathfrak{g}$ (i.e., $t=\sum e_{a} \otimes e_{a}$, where $\left\{e_{a}\right\}$ is an orthonormal basis in $\left.\mathfrak{g}\right)$ and $t_{V}=\rho_{V} \otimes \rho_{V}(t)$. Notice that $r_{V}(u, v)$ is essentially the Cauchy kernel solving the Mittag-Leffler problem on $\mathbb{C} P_{1}$ with which we started.

Proposition 2.16 The matrix coefficients of $L_{V}(u)$ satisfy the Poisson bracket relations

$$
\left\{L_{V}(u) \otimes, L_{V}(v)\right\}=\left[r_{V}(u, v), L_{V}(u) \otimes 1+1 \otimes L_{V}(v)\right] .
$$

We shall now specialize corollary 2.4 to the present setting; it shows that in some way spectral invariants of the Lax operator $L(z)$ may be regarded as 'radial parts' of the Casimir elements.

Remark 2.6 One should use some caution, as Casimir elements do not lie in the symmetric algebra $\mathcal{S}\left(\mathfrak{g}_{D}\right)$ itself but rather in its appropriate local completion; however, their projections to $\mathcal{S}\left(\mathfrak{g}^{N}\right)$ are well defined. More precisely, for each $z_{i} \in D$ consider the projective limit

$$
\tilde{\mathcal{S}}\left(\mathfrak{g}_{z_{i}}\right)=\overleftarrow{\lim }_{n \rightarrow+\infty} \mathcal{S}\left(\mathfrak{g}_{z_{i}}\right) / \mathcal{S}\left(\mathfrak{g}_{z_{i}}\right)\left(\mathfrak{g} \otimes z_{i}^{n} \mathbb{C}\left[\left[z_{i}\right]\right]\right)
$$

and put $\tilde{\mathcal{S}}\left(\mathfrak{g}_{D}\right)=\otimes_{i} \tilde{\mathcal{S}}\left(\mathfrak{g}_{z_{i}}\right)$. Passing to the dual language let us observe that the inner product on $\mathfrak{g}_{D}$ induces the evaluation map $\mathfrak{g}(D) \times \mathcal{S}\left(\mathfrak{g}_{D}\right) \rightarrow \mathbb{C} ;$ the completion is chosen in such a way that this map makes sense for $\tilde{\mathcal{S}}\left(\mathfrak{g}_{D}\right)$. To relate Casimir elements lying in $\tilde{\mathcal{S}}\left(\mathfrak{g}_{D}\right)$ to the more conventional spectral invariants, let us notice that if $L(z) \in \mathfrak{g}(D)$ is a 'Lax matrix', its spectral invariants, e.g., the coefficients of its characteristic polynomial

$$
P(z, \lambda)=\operatorname{det}(L(z)-\lambda)=\sum \sigma_{k}(z) \lambda^{k},
$$

are rational functions in $z$. To get numerical invariants we may expand $\sigma_{k}(z)$ in a local parameter (at any point $\zeta \in \mathbb{C} P_{1}$ ) and take any coefficient of this expansion. The resulting functionals 
are well defined as polynomial mappings from $\mathfrak{g}(D)$ into $\mathbb{C}$. It is easy to see that any such functional is obtained by applying the evaluation map to an appropriate Casimir element $\zeta \in$ $\tilde{\mathcal{S}}\left(\mathfrak{g}_{D}\right)^{\mathfrak{g}_{D}}$.

Clearly, we have $\mathfrak{g}_{D}=\mathfrak{g}(D) \dot{+} \mathfrak{g}^{N} \dot{+} \mathfrak{g}_{D}^{++}$, and hence

$$
\mathcal{S}\left(\mathfrak{g}_{D}\right)=\mathcal{S}\left(\mathfrak{g}^{N}\right) \oplus\left(\mathfrak{g}(D) \mathcal{S}\left(\mathfrak{g}_{D}\right)+\mathcal{S}\left(\mathfrak{g}_{D}\right) \mathfrak{g}_{D}^{++}\right) .
$$

Let $\gamma: \mathcal{S}\left(\mathfrak{g}_{D}\right) \rightarrow \mathcal{S}\left(\mathfrak{g}^{N}\right)$ be the projection map associated with this decomposition.

Proposition 2.17 The restriction of $\gamma$ to the subalgebra $\widetilde{\mathcal{S}}\left(\mathfrak{g}_{D}\right)^{\mathfrak{g}_{D}}$ of Casimir elements is a morphism of Poisson algebras; under the natural pairing $\mathcal{S}\left(\mathfrak{g}^{N}\right) \times \mathfrak{g}(D)_{1} \rightarrow \mathbb{C}$ induced by the inner product (2.25) the restricted Casimirs coincide with the spectral invariants of $L(z)$.

The mapping $\gamma$ is an analogue of the Harish-Chandra homomorphism (Dixmier (1974)); its definition may be extended to the quantum case as well.

Corollary 2.18 Spectral invariants of $L(z)$ are in involution with respect to the standard LiePoisson bracket on $\mathfrak{g}^{N} ; L(z)$ defines a Lax representation for any of these invariants (regarded as a Hamiltonian on $\left(\mathfrak{g}^{N}\right)^{*} \simeq \mathfrak{g}^{N}$.)

The generalized Gaudin Hamiltonians are, by definition, the quadratic Hamiltonians contained in this family; one may take, e.g.,

$$
\mathcal{H}_{V}(u)=\frac{1}{2} \operatorname{tr}_{V} L(u)^{2}
$$

(Physically, they describe, e.g., the bilinear interaction of several 'magnetic momenta'.)

Corollary 2.18 does not mention the 'global' Lie algebra $\mathfrak{g}_{D}$ (and may in fact be proved by elementary means). However, of the three Lie algebras involved, it is probably the most important one, as it is responsible for the dynamics of the system. It is this 'global' Lie algebra that may be called the hidden symmetry algebra.

Setting $P(z, \lambda)=0$ in (2.29), we get an affine algebraic curve (more precisely, a family of curves parametrized by the values of commuting Hamiltonians); this switches on the powerful algebraic-geometric machinery and makes the complete integrability of the generalized Gaudin Hamiltonians almost immediate (Reyman and Semenov-Tian-Shansky (1994)).

Remark 2.7 In the exposition above we kept the divisor D fixed. A more invariant way is of course to use adèlic language. Thus one may replace the algebra $\mathfrak{g}_{D}$ with the global algebra $\mathfrak{g}_{A}$ defined over the ring $\mathbf{A}$ of adèles of $\mathbb{C}(z)$; fixing a divisor amounts to fixing a Poisson subspace inside the Lie algebra $\mathfrak{g}(z)$ of rational functions (which is canonically identified with the dual space of the subalgebra $\mathfrak{g}_{\mathbf{A}}^{+}$). The use of adèlic freedom allowing us to add new points to the divisor is essential in the treatment of the quantum Gaudin model. 
Remark 2.8 Further generalizations consist in replacing the rational $r$-matrix with more complicated ones. One is of course tempted to repeat the construction above, replacing $\mathbb{C} P_{1}$ with an arbitrary algebraic curve. There are obvious obstructions which come from the MittagLeffler theorem for curves: the subalgebra $\mathfrak{g}(C)$ of rational functions on the curve $C$ does not admit a complement in $\mathfrak{g}_{\mathbf{A}}$ which is again a Lie subalgebra; for elliptic curves (and $\mathfrak{g}=$ sl $(n))$ this problem may be solved (Belavin and Drinfeld (1983), Reyman and Semenov-TianShansky (1994)) by considering quasiperiodic functions on $C$, and in this way we recover elliptic r-matrices (which were originally the first example of classical $r$-matrices ever considered (Sklyanin (1979)))!

\section{Quadratic Case}

The integrable systems which we have considered so far are modelled on Poisson submanifolds of linear spaces, namely on the dual spaces of appropriate Lie algebras equipped with the LiePoisson bracket. As we shall see, quantization of such systems (whenever possible) still leaves us in the realm of ordinary (i.e., non-quantum) Lie groups and algebras. Let us note in passing that, in the context of the Integrability phenomena, the name 'Quantum Groups' leads to some confusion: one is tempted to believe that quantization of integrable systems implies that the corresponding 'hidden symmetry groups' also become quantum. As a matter of fact, the real reason to introduce Quantum groups is different. (This is clear from the fact that the Planck constant and the deformation parameter $q$ which enters the definition of Quantum groups are independent of each other!) The point is that we are usually interested not in individual integrable systems, but rather in families of such systems with an arbitrary number of 'particles'. (This is particularly natural for problems arising in Quantum Statistical Mechanics.) A good approximation is obtained if one assumes that the phase space of a multiparticle system is the direct product of phase spaces for single particles. However, the definition of observables for multiparticle systems is not straightforward and tacitly assumes the existence of a Hopf structure (or some of its substitutes) on the algebra $\mathcal{A}$ of observables, i.e. of a map $\Delta^{(N)}: \mathcal{A} \rightarrow$ $\otimes^{N} \mathcal{A}$ which embeds the algebra of observables of a single particle into that of the multiparticle system and thus allows us to speak of individual particles inside the complex system. For systems which are modelled on dual spaces of Lie algebras the underlying Hopf structure is that of $\mathcal{S}(\mathfrak{g})$ or $\mathcal{U}(\mathfrak{g})$ which is derived from the additive structure on $\mathfrak{g}^{*}$ (cf. section 2). There is an important class of integrable systems (difference Lax equations) for which the natural Hopf structure is different; it is derived from the multiplicative structure in a nonabelian Lie group. Let me briefly recall the corresponding construction.

As already mentioned, difference Lax equations arise when the 'auxiliary linear problem' which underlies the construction is associated with a difference equation. A typical example is a first order difference system,

$$
\psi_{n+1}=L_{n} \psi_{n}, n \in \mathbb{Z}
$$

The discrete variable $n \in \mathbb{Z}$ labels the points of an infinite 1-dimensional lattice; if we impose the periodic boundary condition $L_{n}=L_{n+N}$, it is replaced with a finite periodic lattice 
parametrized by $\mathbb{Z} / N \mathbb{Z}$. It is natural to assume that the Lax matrices $L_{n}$ belong to a matrix Lie group $G$. Difference Lax equations arise as compatibility conditions for the linear system,

$$
\begin{aligned}
& \psi_{n+1}=L_{n} \psi_{n}, \\
& \partial_{t} \psi_{n}=A_{n} \psi_{n},
\end{aligned}
$$

with an appropriately chosen $A_{n}$. They have the form of finite-difference zero-curvature equations,

$$
\partial_{t} L_{n}=L_{n} A_{n+1}-A_{n} L_{n} .
$$

Let $\Psi$ be the fundamental solution of the difference system (3.1) normalized by $\Psi_{0}=I$. The value of $\Psi$ at $n=N$ is called the monodromy matrix for the periodic problem and is denoted by $M_{L}$. Clearly,

$$
\begin{aligned}
& \Psi_{n}=\overleftarrow{\prod}_{0 \leq k<n} L_{k}, \\
& M_{L}=\coprod_{0 \leq k<N} L_{k} \text {. }
\end{aligned}
$$

Spectral invariants of the difference operator associated with the periodic difference system (3.1) are the eigenvalues of the monodromy matrix. Thus one expects

$$
I_{s}=\operatorname{tr} M_{L}^{s}, s=1,2, \ldots,
$$

to be the integrals of motion for any Lax equation associated with the linear problem (3.1). This will hold if the monodromy matrix itself evolves isospectrally, i.e. ,

$$
\partial_{t} M_{L}=\left[M_{L}, A\left(M_{L}\right)\right]
$$

for some matrix $A\left(T_{L}\right)$. Let us now discuss possible choices of the Poisson structure on the phase space which will turn (3.3) into Hamiltonian equations of motion. Observe first of all that the phase space of our system is the product $\mathbb{G}=\prod^{N} G$; if we assume that the variables corresponding to different copies of $G$ are independent of each other, we may equip $\mathbb{G}$ with the product Poisson structure. The monodromy may be regarded as a mapping $M: \mathbb{G} \rightarrow G$ which assigns to a sequence $\left(L_{0}, \ldots, L_{N-1}\right)$ the ordered product $M_{L}=\overleftarrow{\prod} L_{k}$. Let $\mathbf{F}_{t}: \mathbb{G} \rightarrow \mathbb{G}$ be the (local) dynamical flow associated with equation (3.3) and $F_{t}: G \rightarrow G$ the corresponding flow associated with equation (3.4) for the monodromy. We expect the following diagram to be commutative:

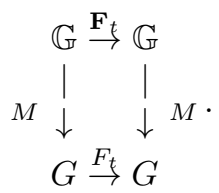

In other words, the dynamics in $\mathbb{G}$ factorizes over $G$. It is natural to demand that all maps in this diagram should be Poissonian; in particular, the monodromy map $M: \mathbb{G} \rightarrow G$ should be compatible with the product structure on $\mathbb{G}=\prod^{N} G$. By induction, this property is reduced to the following one:

Multiplication $m: G \times G \rightarrow G$ is a Poisson mapping. 
This is precisely the axiom introduced by Drinfeld (1983) as a definition of Poisson Lie groups. In brief, one can say that the multiplicativity property of the Poisson bracket on $G$ matches perfectly with the kinematics of one-dimensional lattice systems.

\subsection{Abstract Case: Poisson Lie Groups and Factorizable Lie Bialgebras}

To construct lattice Lax system one may start with an arbitrary Manin triple, or, still more generally, with a factorizable Lie bialgebra. Let us briefly describe this construction which is parallel to the linear case but involves the geometry of Poisson Lie groups. Although main applications are connected with infinite-dimensional groups (e.g., loop groups) it is more instructive to start with the finite-dimensional case.

Let $\left(\mathfrak{g}, \mathfrak{g}^{*}\right)$ be a finite-dimensional factorizable Lie bialgebra. Recall that in this case $r_{ \pm}=$ $\frac{1}{2}(r \pm i d)$ are Lie algebra homomorphisms from $\mathfrak{g}^{*} \simeq \mathfrak{g}_{r}$ into $\mathfrak{g}$. Let $G, G^{*}$ be the connected simply connected Lie groups which correspond to $\mathfrak{g}, \mathfrak{g}^{*}$, respectively. Let us assume that $G$ is a linear group; let $(\rho, V)$ be its faithful linear representation. We extend $r_{ \pm}$to Lie group homomorphisms from $G^{*}$ into $G$ which we denote by the same letters; we shall also write $h_{ \pm}=r_{ \pm}(h), h \in G^{*}$. Both $G$ and $G^{*}$ carry a natural Poisson structure which makes them Poisson Lie groups. The description of the Poisson bracket on $G$ is particularly simple. For $\varphi \in F(G)$ let $D \varphi, D^{\prime} \varphi \in \mathfrak{g}^{*}$ be its left and right differentials defined by

$$
\begin{aligned}
& \langle D \varphi(L), X\rangle=\left.\frac{d}{d t} \varphi(\exp t X \cdot L)\right|_{t=0}, \\
& \left\langle D^{\prime} \varphi(L), X\right\rangle=\left.\frac{d}{d t} \varphi(L \exp t X)\right|_{t=0}, L \in G, X \in \mathfrak{g} .
\end{aligned}
$$

The Sklyanin bracket on $G$ is defined by

$$
\{\varphi, \psi\}=\left\langle r, D^{\prime} \varphi \wedge D^{\prime} \psi\right\rangle-\langle r, D \varphi \wedge D \psi\rangle .
$$

An equivalent description which makes sense when $G$ is a linear group: For $L \in G$ put $L_{V}=$ $\rho_{V}(L)$; the matrix coefficients of $L_{V}$ generate the affine $\operatorname{ring} \mathcal{F}[G]$ of $G$. It is convenient not to fix a representation $\left(\rho_{V}, V\right)$ but to consider all finite-dimensional representations of $G$ simultaneously. The reason is that the Hamiltonians which are used to produce integrable systems are spectral invariants of the Lax matrix. To get a complete set of invariants one may consider either $\operatorname{tr}_{V} L_{V}^{n}$ for a fixed representation and all $n \geq 1$ or, alternatively, $\operatorname{tr}_{V} L_{V}$ for all linear representations. The second version is more convenient in the quantum case. If $\left(\rho_{W}, W\right)$ is another linear representation, we get from the definition (3.6) :

$$
\left\{L_{V} \otimes L_{W}\right\}=\left[r_{V W}, L_{V} \otimes L_{W}\right], \text { where } r_{V W}=\left(\rho_{V} \otimes \rho_{W}\right)(r) .
$$

To avoid excessive use of tensor product signs one usually puts

$$
L_{V}^{1}=L_{V} \otimes I, L_{W}^{2}=I \otimes L_{W}
$$


formula (3.7) is then condensed to

$$
\left\{L_{V}^{1}, L_{W}^{2}\right\}=\left[r_{V W}, L_{V}^{1} L_{W}^{2}\right] .
$$

Let $I \subset \mathcal{F}[G]$ be the subalgebra generated by $\operatorname{tr}_{V} L_{V}, V \in \operatorname{Rep} G$. Formula (3.8) immediately implies the following assertion.

Proposition 3.1 $I \subset \mathcal{F}[G]$ is a commutative subalgebra with respect to the Poisson bracket $(3.6)$.

The dual Poisson bracket on $G^{*}$ may be described in similar terms. Extend $r_{ \pm}$to Lie group homomorphisms $r_{ \pm}: G^{*} \rightarrow G$; it is easy to see that $i_{r}: G^{*} \rightarrow G \times G: T \longmapsto\left(r_{+}(T), r_{-}(T)\right)$ is an injection; hence $G^{*}$ may be identified with a subgroup in $G \times G$. We shall write $r_{ \pm}(T)=$ $T_{ \pm}$for short. Let again $\left(\rho_{V}, V\right),\left(\rho_{W}, W\right)$ be two linear representation of $G$; put $T_{V}^{ \pm}=\rho_{V} \circ$ $T_{ \pm}, T_{W}^{ \pm}=\rho_{W} \circ T_{ \pm} T \in G^{*}$. Matrix coefficients of $T_{V}^{ \pm}, \rho_{V} \in \operatorname{Rep} G$, generate the affine ring $\mathcal{F}\left[G^{*}\right]$ of $G^{*}$.

The Poisson bracket relations for the matrix coefficients of $T_{V}^{ \pm}, T_{W}^{ \pm}$are given by

$$
\begin{aligned}
& \left\{T_{V}^{ \pm 1}, T_{W}^{ \pm 2}\right\}=\left[r_{V W}, T_{V}^{ \pm 1} T_{W}^{ \pm 2}\right] \\
& \left\{T_{V}^{+1}, T_{W}^{-2}\right\}=\left[r_{V W}+t_{V W}, T_{V}^{+1} T_{W}^{-2}\right] ;
\end{aligned}
$$

here $t \in \mathfrak{g} \otimes \mathfrak{g}$ is the tensor Casimir which corresponds to the inner product on $\mathfrak{g}$ and $t_{V W}=$ $\rho_{V} \otimes \rho_{W}(t)$.

Consider the action

$$
G^{*} \times G \rightarrow G:(h, x) \longmapsto h_{+} x h_{-}^{-1} .
$$

Lemma 3.2 $G^{*}$ acts locally freely on an open cell in $G$ containing unit element.

In other words, almost all elements $x \in G$ admit a decomposition $x=h_{+} h_{-}^{-1}$, where $h_{ \pm}=r_{ \pm}(h)$ for some $h \in G^{*}$. (Moreover, this decomposition is unique if both $h$ and $\left(h_{+}, h_{-}\right)$ are sufficiently close to the unit element.) This explains the term 'factorizable group'.

Lemma 3.2 allows us to push forward the Poisson bracket from $G^{*}$ to $G$. More precisely, we have the following result.

Proposition 3.3 There exists a unique Poisson structure $\{,\}^{*}$ on $G$ such that

$$
F: G^{*} \rightarrow G:\left(x_{+}, x_{-}\right) \longmapsto x_{+} x_{-}^{-1}
$$

is a Poisson mapping; it is given by

$$
\begin{aligned}
& \{\varphi, \psi\}^{*}=\langle r, D \varphi \wedge D \psi\rangle+\left\langle r, D^{\prime} \varphi \wedge D^{\prime} \psi\right\rangle \\
& \quad-\left\langle 2 r_{+}, D \varphi \wedge D^{\prime} \psi\right\rangle-\left\langle 2 r_{-}, D^{\prime} \varphi \wedge D \psi\right\rangle, \varphi, \psi \in C^{\infty}(G) .
\end{aligned}
$$

Equivalent description of the bracket $\{,\}^{*}$ for linear groups: let again $L_{V}=\rho_{V}(L), L_{W}=$ $\rho_{W}(L) ;$ then

$$
\begin{aligned}
& \left\{L_{V}^{1}, L_{W}^{2}\right\}^{*}= \\
& r_{V W} L_{V}^{1} L_{W}^{2}+L_{V}^{1} L_{W}^{2} r_{V W} \\
& -L_{V}^{1} r_{V W} L_{W}^{2}-L_{W}^{2} r_{V W} L_{V}^{1}-L_{V}^{1} t_{V W} L_{W}^{2}+L_{W}^{2} t_{V W} L_{V}^{1} .
\end{aligned}
$$


Remark 3.1 (i) A priori the bracket $\{,\}^{*}$ is defined only on the big cell in $G$ (i.e., on the image of $G^{*}$ ); however, formulae (3.11,3.12) show that it extends smoothly to the whole G. (ii) One may notice that the choice of $F$ is actually rigid: this is essentially the only combination of $x_{+}, x_{-}$such that the bracket for $x=F\left(x_{+}, x_{-}\right)$is expressed in terms of $x$ (not of its factors).

Recall that $I \subset \mathcal{F}[G]$ consists of spectral invariants; thus we are again in the setting of the generalized Kostant-Adler theorem: there are two Poisson structures on the same underlying space and the Casimirs of the former are in involution with respect to the latter.

Theorem 3.4 Hamiltonians $\mathcal{H} \in I$ generate Lax equations on $G$ with respect to the Sklyanin bracket .

Geometrically, this means that the Hamiltonian flow generated by $\mathcal{H} \in I$ preserves two systems of symplectic leaves in $G$, namely, the symplectic leaves of $\{$,$\} and \{,\}^{*}$. The latter coincide with the conjugacy classes in $G$ (for a proof see jjciteCIMPA). In order to include lattice Lax equations into our general framework we have to introduce the notion of twisting which is discussed in the next section.

Remark 3.2 The Hamiltonian reduction picture discussed in section 2.1.1 may be fully generalized to the present case as well (Semenov-Tian-Shansky (1994b)). To define the symplectic induction functor we now need the nonabelian moment map (see Reyman (1996) for a review). In the present exposition we shall not describe this construction.

\subsection{Duality theory for Poisson Lie groups and twisted spectral invariants}

Our key observation so far has been the duality between the Hamiltonians of integrable systems on a Lie group and the Casimir functions of its Poisson dual. In section 4.2 we shall see that a similar relation holds for quantized universal enveloping algebras. In order to keep the parallel between the two cases as close as possible we must introduce into the picture still another ingredient: the twisted Poisson structure on the dual group. This notion will also allow us to include lattice Lax equations into our general framework. Roughly speaking, twisting is possible whenever there is an automorphism $\tau \in A u t \mathfrak{g}$ which preserves the r-matrix (i.e., $\tau \otimes$ $\tau \cdot r=r)$. Outer automorphisms are particularly interesting. However, even in the case of inner automorphisms the situation does not become completely trivial. The natural explanation of twisting requires the full duality theory which involves the notions of the double, the Heisenberg double and the twisted double of a Poisson Lie group (Semenov-Tian-Shansky (1994c)). In the present exposition we shall content ourselves by presenting the final formulae for the twisted Poisson brackets. The structure of these formulae is fairly uniform; we list them starting with the case of finite-dimensional simple Lie algebras (where all automorphisms are inner) and then pass to the lattice case (which accounts for the treatment of Lax equations on the lattice) and to the case of affine Lie algebras. In the quantum case which will be considered in the next section twisting will play an important role in the description of quantum Casimirs. 
3.2.1. Finite dimensional simple Lie groups. We begin with a finite dimensional example and return to the setting of section 2.2.2. Let again $\mathfrak{g}$ be a complex simple Lie algebra, $\mathfrak{b}_{+}=$ $\mathfrak{a}+\mathfrak{n}_{+}$its Borel subalgebra, $\mathfrak{b}_{-}$the opposite Borel subalgebra, and $\pi_{ \pm}: \mathfrak{b}_{ \pm} \rightarrow \mathfrak{a}$ the canonical projection,

$$
\mathfrak{g}^{*}=\left\{\left(X_{+}, X_{-}\right) \in \mathfrak{b}_{+} \oplus \mathfrak{b}_{-} \subset \mathfrak{g} \oplus \mathfrak{g} ; \pi_{+}\left(X_{+}\right)=-\pi_{-}\left(X_{-}\right)\right\} .
$$

Let $B_{ \pm} \subset G$ be the corresponding Borel subgroups, and $\pi_{ \pm}: B_{ \pm} \rightarrow A$ the canonical projections,

$$
G^{*}=\left\{\left(x_{+}, x_{-}\right) \in B_{+} \times B_{-} ; \pi_{+}\left(x_{+}\right)=\pi_{-}\left(x_{-}\right)^{-1}\right\} .
$$

Define $r_{d} \in \bigwedge^{2}(\mathfrak{g} \oplus \mathfrak{g})$ by

$$
r_{d}=\left(\begin{array}{cc}
r & -r-t \\
-r+t & r
\end{array}\right)
$$

where $t \in \mathfrak{g} \otimes \mathfrak{g}$ is the tensor Casimir. (One may notice that $r_{d}$ equips $\mathfrak{g} \oplus \mathfrak{g}$ with the structure of a Lie bialgebra which coincides, up to an isomorphism, with that of the Drinfeld double of g.) Our next assertion specializes the results stated in the previous section. Let us define the Poisson bracket on $G^{*}$ by the formula

$$
\{\varphi, \psi\}=\left\langle\left\langle r_{d}, D^{\prime} \varphi \wedge D^{\prime} \psi\right\rangle\right\rangle-\left\langle\left\langle r_{d}, D \varphi \wedge D \psi\right\rangle\right\rangle,
$$

where $D \varphi=\left(\begin{array}{c}D_{+} \varphi \\ D_{-} \varphi\end{array}\right), D^{\prime} \varphi=\left(\begin{array}{c}D_{+}^{\prime} \varphi \\ D_{-}^{\prime} \varphi\end{array}\right)$ are left and right differentials of $\varphi$ with respect to its two arguments and $\langle\langle\rangle$,$\rangle is the natural coupling between \Lambda^{2}(\mathfrak{g} \oplus \mathfrak{g})$ and its dual.

Proposition 3.5 (i) $G^{*}$ is the dual Poisson Lie group of $G$ which corresponds to the standard $r$-matrix described in (2.20). (ii) The mapping $G^{*} \rightarrow G:\left(x_{+}, x_{-}\right) \longmapsto x_{+} x_{-}^{-1}$ is a bijection of $G^{*}$ onto the 'big Schubert cell' in $G$. (iii) The dual Poisson bracket on $G^{*}$ extends smoothly from the big cell to the whole of $G$; it is explicitly given by (3.11) and its Casimirs are central functions on $G$.

The next definition prepares the introduction of twisting.

Definition 3.1 Let $\left(\mathfrak{g}, \mathfrak{g}_{r}\right)$ be a factorizable Lie bialgebra with the classical r-matrix $r \in \mathfrak{g} \otimes \mathfrak{g}$. An automorphism of $\left(\mathfrak{g}, \mathfrak{g}_{r}\right)$ is an automorphism $\varphi \in$ Aut $\mathfrak{g}$ which preserves the r-matrix and the inner product on $\mathfrak{g}$.

We have the following simple result.

Proposition 3.6 Let $\mathfrak{g}$ be a finite-dimensional simple Lie algebra with the standard classical $r$-matrix. Then Aut $\left(\mathfrak{g}, \mathfrak{g}_{r}\right)$ coincides with the Cartan subgroup $A \subset G$.

The action of $A$ on $\wedge^{2} \mathfrak{g}$ is the restriction to $A \subset G$ of the wedge square of the standard adjoint action.

Remark 3.3 If $\mathfrak{g}$ is only semisimple, Aut $\left(\mathfrak{g}, \mathfrak{g}_{r}\right)$ may have a nontrivial group of components. 
The Sklyanin bracket on $G$ is invariant with respect to the action $A \times G \rightarrow G$ by right translations. By contrast, the dual bracket is not invariant, and in this way we get a family of Poisson structures on $G^{*}$ with different Casimir functions. More precisely, define the action $A \times G^{*} \rightarrow G^{*}$ by

$$
h:\left(x_{+}, x_{-}\right) \longmapsto\left(h x_{+}, h^{-1} x_{-}\right)
$$

(Note that this action is compatible with (3.13); the flip $h \longmapsto h^{-1}$ is possible, since $A$ is abelian.) We denote by $\lambda_{h}$ the contragredient action of $h$ on $\mathcal{F}\left[G^{*}\right]: \lambda_{h} \varphi\left(x_{+}, x_{-}\right)=$ $\varphi\left(h^{-1} x_{+}, h x_{-}\right)$. Define the twisted Poisson bracket $\{,\}_{h}^{*}$ on $G^{*}$ by

$$
\{\varphi, \psi\}_{h}^{*}=\lambda_{h}^{-1}\left\{\lambda_{h} \varphi, \lambda_{h} \psi\right\}
$$

Explicitly we get

$$
\{\varphi, \psi\}_{h}^{*}=\left\langle\left\langle r_{d}^{h} D^{\prime} \varphi, D^{\prime} \psi\right\rangle\right\rangle-\left\langle\left\langle r_{d} D \varphi, D \psi\right\rangle\right\rangle
$$

where

$$
r_{d}^{h}=\bigwedge^{2}\left(A d h \oplus A d h^{-1}\right) \cdot r_{d}=\left(\begin{array}{cc}
r & (-r-t)^{h} \\
(-r+t) & h^{-1} \\
r
\end{array}\right),
$$

or, in matrix form,

$$
\begin{aligned}
& \left\{T_{V}^{ \pm 1}, T_{W}^{ \pm 2}\right\}_{h}^{*}=\left[r_{V W}, T_{V}^{ \pm 1} T_{W}^{ \pm 2}\right], \\
& \left\{T_{V}^{+1}, T_{W}^{-2}\right\}_{h}^{*}=\left(r_{V W}^{h}+t_{V W}^{h}\right) T_{V}^{+1} T_{W}^{-2}-T_{V}^{+1} T_{W}^{-2}\left(r_{V W}+t_{V W}\right),
\end{aligned}
$$

where $r_{V W}^{h}=A d h \otimes A d h^{-1} \cdot r_{V W}=A d h^{2} \otimes i d \cdot r_{V W}$ and similarly for $t_{V W}^{h}$.

Let us denote by $I_{h}(G)$ the set of twisted spectral invariants; by definition, $\phi \in I_{h}(G)$ if

$$
\phi(g x)=\phi(x g h) \text { for any } x, g \in G \text {. }
$$

Example 3.1 Let $(\rho, V)$ be a linear representation of $G$; then $\phi: x \longmapsto \operatorname{tr}_{V} \rho(h x)$ is a twisted spectral invariant.

Proposition 3.7 (i) There exists a unique Poisson structure on $G$ such that the twisted factorization map $F_{h}: G^{*} \rightarrow G:\left(x_{+}, x_{-}\right) \longmapsto h^{2} x_{+} x_{-}$is a Poisson mapping. (ii) $F_{h}$ transforms the Casimir functions of $\{,\}_{h}^{*}$ into the set of twisted spectral invariants $I_{h^{2}}(G)$. (ii) Twisted spectral invariants also commute with respect to the Sklyanin bracket on $G$ and generate on $G$ generalized Lax equations

$$
\frac{d L}{d t}=A L-L B, A=A d h^{2} \cdot B
$$


It is instructive to write an explicit formula for the bracket on $G$ which is the push-forward of $\{,\}_{h}^{*}$ with respect to the factorization map. We get

$$
\begin{aligned}
& \{\varphi, \psi\}_{h}^{*}=\langle r, D \varphi \wedge D \psi\rangle+\left\langle r, D^{\prime} \varphi \wedge D^{\prime} \psi\right\rangle \\
& \quad-\left\langle r^{h}, D \varphi \wedge D^{\prime} \psi\right\rangle-\left\langle r^{h^{-1}}, D^{\prime} \varphi \wedge D \psi\right\rangle \\
& -\left\langle t^{h}, D \varphi \wedge D^{\prime} \psi\right\rangle+\left\langle t^{h^{-1}}, D^{\prime} \varphi \wedge D \psi\right\rangle, \varphi, \psi \in C^{\infty}(G)
\end{aligned}
$$

where $t$ is the tensor Casimir, and we put $r^{h}=A d h \otimes \cdot A d h^{-1} \cdot r$ and $t^{h}=A d h \otimes A d h^{-1 j} \cdot t$. This bracket again extends smoothly to the whole of $G$ (cf. (3.11). Equivalently,

$$
\begin{aligned}
\left\{L_{V}^{1}, L_{W}^{2}\right\}_{h}^{*}= & r_{V W} L_{V}^{1} L_{V}^{2}+L_{V}^{1} L_{W}^{2} r_{V W} \\
& -L_{V}^{1} r_{V W}^{h} L_{V}^{2}-L_{W}^{2} r_{V W}^{h^{-1}} L_{V}^{1} \\
& -L_{V}^{1} t_{V W}^{h} L_{W}^{2}+L_{W}^{2} t_{V W}^{h^{-1}} L_{V}^{1}
\end{aligned}
$$

The twisted bracket $\{,\}_{h}^{*}$ on $G^{*}$ is not multiplicative with respect to the group structure on $G^{*}$; to explain its relation to the Poisson group theory we shall need some more work.

Let $\mathfrak{a} \ltimes \mathfrak{g}^{*}$ be the semidirect product of Lie algebras which corresponds to action (3.16); in other words, we set

$$
\left[H,\left(X_{+}, X_{-}\right)\right]=\left(\left[H, X_{+}\right],-\left[H, X_{-}\right]\right), H \in \mathfrak{a}, X_{ \pm} \in \mathfrak{b}_{ \pm}
$$

Fix a basis $\left\{H_{i}\right\}$ of $\mathfrak{a}$ and let $\left\{H^{i}\right\}$ be the dual basis of $\mathfrak{a}^{*}$. We define a (trivial) 2-cocycle on $\mathfrak{g}$ with values in $\mathfrak{a}^{*}$ by

$$
\omega(X, Y)=\sum_{i}\left(H_{i},[X, Y]\right) H^{i} .
$$

Let $\widehat{\mathfrak{g}}=\mathfrak{g} \oplus \mathfrak{a}^{*}$ be the central extension of $\mathfrak{g}$ by $\mathfrak{a}^{*}$ which corresponds to this cocycle.

Proposition $3.8\left(\mathfrak{a} \ltimes \mathfrak{g}^{*}, \widehat{\mathfrak{g}}\right)$ is a Lie bialgebra.

The Poisson Lie group which corresponds to $\mathfrak{a} \ltimes \mathfrak{g}^{*}$ is the semidirect product $A \ltimes G^{*}$ associated with action (3.16). It is easy to see that the variable $h \in A$ is central with respect to this bracket; thus the bracket on $A \ltimes G^{*}$ splits into a family of brackets on $G^{*}$ parametrized by $h \in A$. This is precisely the family $\{,\}_{h}^{*}$.

3.2.2.Twisting on the lattice. One may notice that the previous example is in fact trivial, since the twisted bracket differs from the original one by a change of variables ${ }^{1}$. This is of course closely related to the fact that the central extension associated with the cocycle (3.21) is also trivial. Our next example is more interesting; it is adapted to the study of Lax equations on the lattice. Put $\Gamma=\mathbb{Z} / N \mathbb{Z}$. Let again $\mathfrak{g}$ be a finite dimensional simple Lie algebra equipped with a standard r-matrix. Let $\mathrm{g}=\mathfrak{g}^{\Gamma}$ be the Lie algebra of functions on $\Gamma$ with values in $\mathfrak{g}$; obviously,

\footnotetext{
${ }^{1}$ However, even in this case twisted spectral invariants give rise to a different set of Hamiltonians, as compared with the non-twisted case.
} 
$\mathrm{g}$ inherits from $\mathfrak{g}$ the natural Lie bialgebra structure; the corresponding $r$-matrix $r \in \Lambda^{2} \mathrm{~g}$ is given by $\mathrm{r}_{m n}=\delta_{m n} r, m, n \in \Gamma$. We define $\mathrm{r}_{d} \in \bigwedge^{2}(\mathrm{~g} \oplus \mathrm{g})$ by

$$
r_{d}=\left(\begin{array}{cc}
r & -r-t \\
-r+t & r
\end{array}\right)
$$

where $\mathrm{t} \in \mathrm{g} \otimes \mathrm{g}$ is the tensor Casimir which corresponds to the standard inner product on $\mathrm{g}$,

$$
\langle\langle X, Y\rangle\rangle=\sum_{n}\left\langle X_{n}, Y_{n}\right\rangle .
$$

Let $\tau$ be the cyclic permutation on $\mathrm{g},(\tau X)_{n}=X_{n+1 \bmod N}$.

Lemma $3.9 \tau \in A u t\left(\mathrm{~g}, \mathrm{~g}^{*}\right)$.

Let us extend the action of $\tau$ to $\mathrm{g} \oplus \mathrm{g}$ in the following way:

$$
\tau \cdot(X, Y)=(\tau X, Y) .
$$

(By analogy with (3.16), one might rotate the second copy of $g$ in the opposite sense, but then the total shift in formulae below would be by two units, which is less natural on the lattice.) Put

$$
\begin{aligned}
& \mathrm{r}_{d}^{\tau}=\bigwedge^{2}(\tau \oplus i d) \cdot \mathrm{r}_{d}=\left(\begin{array}{cc}
\mathrm{r} & -\mathrm{r}^{\tau}-\mathrm{t}^{\tau} \\
-\mathrm{r}^{\tau^{-1}}+\mathrm{t}^{\tau^{-1}} & \mathrm{r}
\end{array}\right), \\
& \mathrm{r}^{\tau}=\tau \otimes i d \cdot \mathrm{r}, \mathrm{r}^{\tau^{-1}}=\tau^{-1} \otimes i d \cdot \mathrm{r}=i d \otimes \tau \cdot \mathrm{r} .
\end{aligned}
$$

Put $\mathbb{G}=G^{\Gamma}, \mathbb{G}^{*}=G^{*} \Gamma$. The action of $\tau$ extends to $\mathbb{G}$ and to $\mathbb{G}^{*}$ in an obvious way. As in section 3.2.1 we may embed $\mathbb{G}^{*}$ into the direct product $\mathbb{G} \times \mathbb{G}$. By definition, the twisted Poisson bracket on $\mathbb{G}^{*}$ is given by

$$
\{\varphi, \psi\}_{\tau}^{*}=\left\langle\left\langle\mathrm{r}_{d}^{\tau}, D \varphi \wedge D \psi\right\rangle\right\rangle-\left\langle\left\langle\mathrm{r}_{d} D^{\prime} \varphi \wedge D^{\prime} \psi\right\rangle\right\rangle,
$$

where $D \varphi, D^{\prime} \varphi \in \mathrm{g} \oplus \mathrm{g}$ are the two-component left and right differentials of $\varphi$.

We may push forward the Poisson bracket from $\mathbb{G}^{*}$ to $\mathbb{G}$ using the factorization map. More precisely:

Proposition 3.10 There exists a unique Poisson structure on $\mathbb{G}$ (which we shall still denote by $\left.\{,\}_{\tau}^{*}\right)$ such that the twisted factorization map $\mathbb{G}^{*} \rightarrow \mathbb{G}:\left(x_{+}, x_{-}\right) \longmapsto x_{+}^{\tau} x_{-}^{-1}$ becomes a Poisson mapping. This Poisson structure is given by

$$
\begin{aligned}
& \{\varphi, \psi\}_{\tau}^{*}=\langle r, D \varphi \wedge D \psi\rangle+\left\langle r, D^{\prime} \varphi \wedge D^{\prime} \psi\right\rangle \\
& \quad-\left\langle r^{\tau}, D \varphi \wedge D^{\prime} \psi\right\rangle-\left\langle r^{\tau^{-1}}, D^{\prime} \varphi \wedge D \psi\right\rangle \\
& -\left\langle t^{\tau}, D \varphi \wedge D^{\prime} \psi\right\rangle+\left\langle t^{\tau^{-1}}, D^{\prime} \varphi \wedge D \psi\right\rangle, \varphi, \psi \in C^{\infty}(\mathbb{G}),
\end{aligned}
$$


We shall denote the Lie group $\mathbb{G}$ equipped with the Poisson structure $(3.23)$ by $\mathbb{G}_{\tau}$, for short. Equivalent description of the bracket $\{,\}_{\tau}^{*}$ for linear groups: let again $L_{V}=\rho_{V}(L)$; then

$$
\begin{aligned}
\left\{L_{V}^{1}, L_{V}^{2}\right\}^{*}= & r L_{V}^{1} L_{V}^{2}+L_{V}^{1} L_{V}^{2} r-L_{V}^{1} r^{\tau} L_{V}^{2}-L_{V}^{2} r^{\tau^{-1}} L_{V}^{1} \\
& -L_{V}^{1} t^{\tau} L_{V}^{2}-L_{V}^{2} t^{\tau^{-1}} L_{V}^{1}
\end{aligned}
$$

Another obvious Poisson structure on $\mathbb{G}$ is the Sklyanin bracket which corresponds to the Lie bialgebra $\left(\mathrm{g}, \mathrm{g}^{*}\right)$. Let us consider the following action of $\mathbb{G}$ on $\mathbb{G}_{\tau}$ called the (lattice) gauge action:

$$
x: T \longmapsto x^{\tau} T x^{-1} .
$$

Theorem 3.11 (i) The gauge action $\mathbb{G} \times \mathbb{G}_{\tau} \rightarrow \mathbb{G}_{\tau}$ is a Poisson group action. (ii) The Casimir functions of $\{,\}_{\tau}^{*}$ coincide with the gauge invariants. (iii) Let $M: \mathbb{G}_{\tau} \rightarrow G$ be the monodromy map,

$$
M: T=\left(T_{1}, T_{2}, \ldots, T_{N}\right) \longmapsto T_{N} \cdot T_{1} ;
$$

we equip the target space with Poisson structure (3.11). Then $M$ is a Poisson map and the Casimirs of $\mathbb{G}_{\tau}$ coincide with the spectral invariants of the monodromy. (iv) Spectral invariants of the monodromy are in involution with respect to the Sklyanin bracket on $\mathbb{G}$ and generate lattice Lax equations on $\mathbb{G}$.

Parallels with theorem 2.3 are completely obvious.

3.2.3. Twisting for loop algebras. Our last example of twisting is based on the outer automorphisms of loop algebras. We shall use the notation and definitions introduced in section 2.2.2. Let $\mathfrak{g}$ be a simple Lie algebra, $L \mathfrak{g}$ the corresponding loop algebra with the standard trigonometric r-matrix, and let $L \mathfrak{g}_{r}=(L \mathfrak{g})^{*}$ be the dual algebra. The Lie group which corresponds to $L \mathfrak{g}$ is the group $L G$ of polynomial loops with values in $G$; the elements of the dual Poisson group may be identified with pairs of formal series $\left(T^{+}(z), T^{-}\left(z^{-1}\right)\right)$,

$$
T^{ \pm}(z)=\sum_{n \geq 0} T^{ \pm}[ \pm n] z^{ \pm n}, T^{ \pm}[0] \in B_{ \pm}, \pi_{+}\left(T^{+}[0]\right) \cdot \pi_{-}\left(T^{-}[0]\right)=1
$$

(here $\pi_{ \pm}: B_{ \pm} \rightarrow H$ are canonical projections). The group $(L G)^{*}$ is pro-algebraic and its affine ring is generated by the matrix coefficients of $T^{ \pm}[n]$.

The next proposition describes the Poisson structure on the affine ring of $(L G)^{*}$. Let $t \in \mathfrak{g} \otimes \mathfrak{g}$ be the canonical element (tensor Casimir) which represents the inner product $\langle$,$\rangle in \mathfrak{g}$. Let $\delta(x)$ be the Dirac delta,

$$
\delta(x)=\sum_{n=-\infty}^{\infty} x^{n}
$$

(observe that $t \cdot \delta(z / w)$ represents the kernel of the identity operator acting in $L \mathfrak{g}$ ). 
Proposition 3.12 The Poisson bracket on $(L G)^{*}$ which corresponds to the standard Lie bialgebra structure on $L \mathfrak{g}$ is given by

$$
\begin{array}{r}
\left\{T_{1}^{ \pm}(z), T_{2}^{ \pm}(w)\right\}=\left[r\left(\frac{z}{w}\right), T_{1}^{ \pm}(z) T_{2}^{ \pm}(w)\right], \\
\left\{T_{1}^{+}(z), T_{2}^{-}(w)\right\}=\left[r\left(\frac{z}{w}\right), T_{1}^{+}(z) T_{2}^{-}(w)\right]+\delta\left(\frac{z}{w}\right)\left[t, T_{1}^{+}(z) T_{2}^{-}(w)\right],
\end{array}
$$

where $r(x)$ is the trigonometric r-matrix.

We pass to the description of the twisted structure on $(L G)^{*}$.

Lemma 3.13 Aut $\left(L \mathfrak{g}, L \mathfrak{g}_{r}\right)$ is the extended Cartan subgroup $\hat{H}=H \times \mathbb{C}^{\times}$

By definition, $p \in \mathbb{C}^{\times}$acts on $L \mathfrak{g}$ by sending $X(z) \longmapsto X(p z)$; we shall denote the corresponding dilation operator by $D_{p}$. The action of $H$ gives nothing new, as compared with our first example (section 2.2.1). To describe the twisting of the Poisson structure on $(L G)^{*}$ by the action of $\mathbb{C}^{\times}$we start with the r-matrix

$$
r_{d}(z / w)=\left(\begin{array}{cc}
r(z / w) & -r(z / w)-t \delta(z / w) \\
-r(z / w)+t \delta(z / w) & r(z / w)
\end{array}\right)
$$

(as usual, $r_{d}(z / w)$ does not belong to the $\bigwedge^{2}(L \mathfrak{g} \oplus L \mathfrak{g})$, but is well defined as a kernel of a linear operator acting in $L \mathfrak{g} \oplus L \mathfrak{g})$. For $p \in \mathbb{C}^{\times}$we define the twisted r-matrix by

$$
r_{d}(z / w)^{p}=\bigwedge^{2}\left(D_{p} \oplus D_{p}^{-1}\right) \cdot r_{d}(z / w)=\left(\begin{array}{cc}
r(z / w) & -r(p z / w)-t \delta(p z / w) \\
-r(z / p w)+t \delta(z / p w) & r(z / w)
\end{array}\right) .
$$

Definition 3.2 The twisted Poisson bracket on $(L G)^{*}$ is given by

$$
\begin{array}{r}
\left\{T_{1}^{ \pm}(z), T_{2}^{ \pm}(w)\right\}_{p}^{*}=\left[r\left(\frac{z}{w}\right), T_{1}^{ \pm}(z) T_{2}^{ \pm}(w)\right] \\
\left\{T_{1}^{+}(z), T_{2}^{-}(w)\right\}_{p}^{*}=r\left(\frac{p z}{w}\right) T_{1}^{+}(z) T_{2}^{-}(w)-T_{1}^{+}(z) T_{2}^{-}(w) r\left(\frac{z}{w}\right) \\
+\delta\left(\frac{p z}{w}\right) t T_{1}^{+}(z) T_{2}^{-}(w)-T_{1}^{+}(z) T_{2}^{-}(w) \delta\left(\frac{z}{w}\right) t
\end{array}
$$

Define the new generating function ('full current') by

$$
T(z)=T^{+}(p z) T^{-}\left(p^{-1} z\right)^{-1} ;
$$

clearly, the coefficients of $T(z)$ generate the affine ring of $(L G)^{*}$. 
Proposition 3.14 The current $T(z)$ satisfies the following Poisson bracket relations:

$$
\begin{array}{r}
\left\{T_{1}(z), T_{2}(w)\right\}_{p}^{*}= \\
r\left(\frac{z}{w}\right) T_{1}(z) T_{2}(w)+T_{1}(z) T_{2}(w) r\left(\frac{z}{w}\right) \\
-T_{1}(z) r\left(\frac{p^{2} z}{w}\right) T_{2}(w)-T_{2}(w) r\left(\frac{z}{p^{2} w}\right) T_{1}(z) \\
-T_{1}(z) \delta\left(\frac{p^{2} z}{w}\right) t T_{2}(w)+T_{2}(w) \delta\left(\frac{z}{p^{2} w}\right) t T_{1}(z) .
\end{array}
$$

The existence of twisting in the affine case is due to the existence of the non-trivial central extension of the loop algebra. (This should be compared with the situation in our first example, where the central extension is trivial because all automorphisms of simple Lie algebras are inner.) More precisely, we have the following result. Let $\omega$ be the 2-cocycle on $L \mathfrak{g}$ defined by

$$
\omega(X, Y)=\operatorname{Res}_{z=0}\left\langle X(z), z \partial_{z} Y(z)\right\rangle d z / z .
$$

Let $\widehat{L \mathfrak{g}}$ be the central extension of $L \mathfrak{g}$ determined by this cocycle, and $\widehat{L \mathfrak{g}}^{*}$ the semidirect product of $(L \mathfrak{g})^{*}$ and $\mathbb{C}^{\times}$.

Proposition $3.15\left(\widehat{L \mathfrak{g}}, \widehat{L \mathfrak{g}}^{*}\right)$ is a Lie bialgebra.

The Poisson Lie group which corresponds to $\widehat{L \mathfrak{g}}^{*}$ is the semidirect product $\mathbb{C}^{\times} \ltimes(L G)^{*}$; it is easy to see that the affine coordinate on $\mathbb{C}^{\times}$is central with respect to the Poisson structure on $\mathbb{C}^{\times} \ltimes(L G)^{*}$; fixing its value, we get the family of Poisson brackets $\{,\}_{p}^{*}$ on $(L G)^{*}$.

In section 4.2 we shall discuss a similar phenomenon for quantum systems associated with quasitriangular Hopf algebras.

\subsection{Sklyanin Bracket on G $(z)$}

In our previous example we have described the Poisson structure associated with the loop algebra, namely, the bracket on the dual group $(L G)^{*}$; applications to lattice systems involve the Poisson structure on the loop group itself. As a matter of fact, this was the first example of a Poisson Lie group which appeared prior to their formal definition (Sklyanin (1979)). To put it more precisely, let $\mathfrak{g}$ be a complex semisimple Lie algebra, $G$ a linear complex Lie group with the Lie algebra $\mathfrak{g}$. Let $G(z)$ be the associated loop group, i.e., the group of rational functions with values in $G$. We may regard $G(z)$ as an affine algebraic group defined over $\mathbb{C}(z)$. Let us again fix a faithful representation $(\rho, V)$ of $G$; the affine ring $\mathcal{A}_{a f f}$ of $G(z)$ is generated by 'tautological functions' which assign to $L \in G(z)$ the matrix coefficients of $L_{V}(z) \in E n d V \otimes \mathbb{C}(z)$. The Poisson bracket on the affine ring of $G(z)$ is defined by the following formula (Sklyanin (1979)),

$$
\left\{L_{V}^{1}(u), L_{V}^{2}(v)\right\}=\left[r_{V}(u, v), L_{V}^{1}(u) L_{V}^{2}(v)\right] .
$$


(By an abuse of notation, we do not distinguish between the generators of $\mathcal{A}_{a f f}$ and their values at $L \in G(z)$ in the l.h.s. of this formula.) Bracket (3.30) defines the structure of a Poisson-Lie group on $G(z)$. Equivalent formulation:

Let $\Delta: \mathcal{A}_{\text {aff }} \rightarrow \mathcal{A}_{\text {aff }} \otimes \mathcal{A}_{\text {aff }}$ be the coproduct in $\mathcal{A}_{\text {aff }}$ induced by the group multiplication in $G(z)$ :

$$
\Delta\left(L_{V}(z)\right)=L_{V}(z) \dot{\otimes} L_{V}(z),
$$

or, in a less condensed notation,

$$
\Delta\left(L_{V}^{i j}(z)\right)=\sum_{k} L_{V}^{i k}(z) \otimes L_{V}^{k j}(z) .
$$

Then $\Delta$ is a morphism of Poisson algebras (in other words,

$$
\{\Delta \varphi, \Delta \psi\}=\Delta\{\varphi, \psi\}
$$

for any $\left.\varphi, \psi \in \mathcal{A}_{a f f}\right)$.

The Lax matrix $L_{V}(u)$ in this context is basically a tautological mapping which assigns to an element $L \in G(z)$ the matrix $L_{V}(u) \in E n d V \otimes \mathbb{C}(u)$. Alternatively, for any Poisson submanifold $\mathcal{M} \subset G(z)$ the Lax matrix may be regarded as an embedding map $\mathcal{M} \hookrightarrow G(z) \rightarrow$ End $V \otimes \mathbb{C}(u)$. An explicit description of all Poisson submanifolds in $G(z)$ follows from the theory of dressing transformations (Semenov-Tian-Shansky (1994b)); for our present goals it suffices to know that rational functions with a prescribed divisor of poles form a finite-dimensional Poisson submanifold in $G(z)$; moreover, since the Poisson structure is multiplicative, the product of Poisson submanifolds is again a Poisson submanifold. Generic Poisson submanifolds correspond to functions with simple poles which may be written in multiplicative form

$$
L_{V}(z)=\prod_{i}^{\curvearrowleft}\left(I-\frac{X_{i}}{z-z_{i}}\right) .
$$

In other words, the description of Poisson submanifolds is tantamount to the choice of an Ansatz for the Lax matrix; the free parameters in this Ansatz (e.g., the residues $X_{i}$ ) become dynamical variables. Spectral invariants of 'Lax matrices' of this type may be used to generate completely integrable lattice Lax equations. More precisely, to get such system one may proceed as follows:

- Pick a Poisson submanifold $\mathcal{M} \subset G(z)$.

- Consider the product space $\mathcal{M}^{N} \subset G(z)^{\mathbb{Z} / N \mathbb{Z}}$ and the monodromy map $m: G(z) \times \ldots \times$ $G(z) \rightarrow G(z)$ (more precisely, its restriction to $\mathcal{M}^{N}$ ).

- Choose any central function $\mathcal{H}$ on $G(z)$ as a Hamiltonian; its pullback to $\mathcal{M}^{N}$ defines a lattice Lax equation.

Under some mild assumptions on the choice of $\mathcal{M}, \mathcal{H}$ defines a completely integrable system on $m\left(\mathcal{M}^{N}\right)$; its pullback to $\mathcal{M}^{N}$ still remains completely integrable; this may be regarded as the main content of the Inverse Scattering Method (in this slightly simplified setting).

The study of Lax systems on the lattice thus breaks naturally into two parts: (a) Solve the Lax equation for the monodromy. (b) Lift the solutions back to $G(z)^{N}$. The second stage (by no means trivial) is the inverse problem, sensu strictu. 


\section{Quantization}

\subsection{Linear case}

Speaking informally, quantization consists in replacing Poisson bracket relations with commutation relations in an associative algebra. We shall have to distinguish between linear and quadratic cases (as explained in the previous section, this difference stems from the different Hopf structures on the algebras of observables). The linear case is easier, since we remain in the conventional setting of Lie groups and Lie algebras. Quantization of quadratic Poisson bracket relations (3.7) leads to quasitriangular Hopf algebras.

The standard way to quantize the algebra $\mathcal{A}_{c l}=\mathcal{S}(\mathfrak{g})$ (equipped, as usual, with the LiePoisson bracket of $\mathfrak{g}$ ) is to replace it with the universal enveloping algebra $\mathcal{U}(\mathfrak{g})$. Let me briefly recall the corresponding construction.

Let $\mathcal{U}(\mathfrak{g})=\bigcup_{n>0} \mathcal{U}_{n}$ be the canonical filtration of $\mathcal{U}(\mathfrak{g})$, and $\mathcal{S}(\mathfrak{g})=\oplus_{n \geq 0} \mathcal{S}_{n}$ the canonical grading of $\mathcal{S}(\mathfrak{g})$. Clearly, $\mathcal{S}_{n} \simeq \mathcal{U}_{n} / \mathcal{U}_{n-1}$. Let $g r_{n}: \mathcal{U}_{n} \rightarrow \mathcal{S}_{n}$ be the canonical projection. If $u \in \mathcal{U}_{n}, v \in \mathcal{U}_{m}$, their commutator $[u, v] \in \mathcal{U}_{n+m-1}$. It is easy to see that $g r_{n+m-1}([u, v])=$ $\left\{g r_{n} u, g r_{m} v\right\}$ is precisely the Lie-Poisson bracket; hence $\mathcal{U}(\mathfrak{g})$ is a quantization of $\mathcal{S}(\mathfrak{g})$.

Alternatively, let $\hbar$ be a formal parameter. Put $\mathfrak{g}_{\hbar}=\mathfrak{g} \otimes_{k} k[[\hbar]]$; we rescale the commutator in $\mathfrak{g}_{\hbar}$ by putting $[X, Y]_{\hbar}=\hbar[X, Y]$. Let $\mathcal{U}\left(\mathfrak{g}_{\hbar}\right)$ be the universal enveloping algebra of $\mathfrak{g}_{\hbar}$, and $\hbar \mathcal{U}\left(\mathfrak{g}_{\hbar}\right)$ be its maximal ideal consisting of formal series in $\hbar$ with zero constant term; the quotient algebra $\mathcal{U}\left(\mathfrak{g}_{\hbar}\right) / \hbar \mathcal{U}\left(\mathfrak{g}_{\hbar}\right)$ is canonically isomorphic to $\mathcal{S}(\mathfrak{g})$. Let $p: \mathcal{U}\left(\mathfrak{g}_{\hbar}\right) \rightarrow \mathcal{S}(\mathfrak{g})$ be the canonical projection; then $\{p(x), p(y)\}=p\left(\hbar^{-1}(x y-y x)\right)$ for any $x, y \in \mathcal{U}\left(\mathfrak{g}_{\hbar}\right)$. (In the sequel we prefer to set $\hbar=1$ and so the first definition will be more convenient.)

Let $r \in E n d \mathfrak{g}$ be a classical r-matrix. As we have seen, the phase spaces of Lax systems associated with $(\mathfrak{g}, r)$ are coadjoint orbits of $\mathfrak{g}_{r}$; the Hamiltonians are obtained from the Casimir elements $H \in \mathcal{S}(\mathfrak{g})^{\mathfrak{g}}$ via the isomorphism $\mathcal{S}\left(\mathfrak{g}_{r}\right) \rightarrow \mathcal{S}(\mathfrak{g})$ described in theorem 2.3. The quantum counterpart of $\mathcal{S}(\mathfrak{g})^{\mathfrak{g}}$ is the center $\mathcal{Z} \subset \mathcal{U}(\mathfrak{g})$ of the universal enveloping algebra. Moreover, under favorable conditions there is a nice correspondence between coadjoint orbits of a Lie algebra and unitary representations of the corresponding Lie group. Thus we may establish the following heuristic vocabulary which describes the correspondence between classical and quantum systems:

\begin{tabular}{|c|c|}
\hline Linear Classical Case & Linear Quantum case \\
\hline $\mathcal{S}(\mathfrak{g})$ & $\mathcal{U}(\mathfrak{g})$ \\
\hline Classical r-matrices & Classical r-matrices \\
\hline Casimir functions $(\mathcal{S}(\mathfrak{g}))^{\mathfrak{g}} \subset \mathcal{S}(\mathfrak{g})$ & Casimir operators $\mathcal{Z} \subset \mathcal{U}(\mathfrak{g})$ \\
\hline $\mathcal{S}\left(\mathfrak{g}_{r}\right)$ & $\mathcal{U}\left(\mathfrak{g}_{r}\right)$ \\
\hline Coadjoint orbits in $\mathfrak{g}_{r}^{*}$ & Irreducible $\mathcal{U}\left(\mathfrak{g}_{r}\right)$-modules \\
\hline
\end{tabular}

Notice that, in the linear case, classical r-matrices are used in the quantum case as well!

The following theorem is an exact analogue of theorem 2.3

Let $(\mathfrak{g}, r)$ be a Lie algebra equipped with a classical r-matrix $r \in E n d \mathfrak{g}$ satisfying (2.8). Let $\mathfrak{g}_{r}$ be the corresponding Lie algebra (with the same underlying linear space). Put $r_{ \pm}=$ $\frac{1}{2}(r \pm i d)$; then (2.8) implies that $r_{ \pm}: \mathfrak{g}_{r} \rightarrow \mathfrak{g}$ are Lie algebra homomorphisms. Extend them 
to homomorphisms $\mathcal{U}\left(\mathfrak{g}_{r}\right) \rightarrow \mathcal{U}(\mathfrak{g})$ which we denote by the same letters. These morphisms also agree with the standard Hopf structure on $\mathcal{U}(\mathfrak{g}), \mathcal{U}\left(\mathfrak{g}_{r}\right)$. Define the action

$$
\mathcal{U}\left(\mathfrak{g}_{r}\right) \otimes \mathcal{U}(\mathfrak{g}) \rightarrow \mathcal{U}(\mathfrak{g})
$$

by setting

$$
x \cdot y=\sum r_{+}\left(x_{i}^{(1)}\right) y r_{-}\left(x_{i}^{(2)}\right)^{\prime}, x \in \mathcal{U}\left(\mathfrak{g}_{r}\right), y \in \mathcal{U}(\mathfrak{g}),
$$

where $\Delta x=\sum x_{i}^{(1)} \otimes x_{i}^{(2)}$ is the coproduct and $a \mapsto a^{\prime}$ is the antipode map.

Theorem 4.1 (i) $\mathcal{U}(\mathfrak{g})$ is a free filtered Hopf $\mathcal{U}\left(\mathfrak{g}_{r}\right)$-module generated by $1 \in \mathcal{U}(\mathfrak{g})$. (ii) Let $i: \mathcal{U}(\mathfrak{g}) \rightarrow \mathcal{U}\left(\mathfrak{g}_{r}\right)$ be the induced isomorphism of filtered linear spaces; its restriction to $\mathcal{Z} \subset \mathcal{U}(\mathfrak{g})$ is an algebra homomorphism; in particular, $i(\mathcal{Z}) \subset \mathcal{U}\left(\mathfrak{g}_{r}\right)$ is commutative.

Let $\mathfrak{u} \subset \mathfrak{g}_{r}$ be an ideal, $\mathfrak{s}=\mathfrak{g}_{r} / \mathfrak{u}$ the quotient algebra, and $p: \mathcal{U}\left(\mathfrak{g}_{r}\right) \rightarrow \mathcal{U}(\mathfrak{s})$ be the canonical projection; restricting $p$ to the subalgebra $I_{r}=i_{r}(\mathcal{Z})$ we get a commutative subalgebra in $\mathcal{U}(\mathfrak{s})$; we shall say that the corresponding elements of $\mathcal{U}(\mathfrak{s})$ are obtained by specialization.

In particular, let $\mathfrak{g}=\mathfrak{g}_{+}+\mathfrak{g}_{-}$be a splitting of $\mathfrak{g}$ into a linear sum of two its Lie subalgebras; let $P: \mathcal{U}(\mathfrak{g}) \rightarrow \mathcal{U}\left(\mathfrak{g}_{-}\right)$be the projection onto $\mathcal{U}\left(\mathfrak{g}_{-}\right)$in the decomposition

$$
\mathcal{U}(\mathfrak{g})=\mathcal{U}\left(\mathfrak{g}_{-}\right) \oplus \mathfrak{g}_{+} \mathcal{U}(\mathfrak{g}) .
$$

Corollary 4.2 The restriction of $P$ to the subalgebra $\mathcal{Z} \subset \mathcal{U}(\mathfrak{g})$ of Casimir elements is an algebra homomorphism.

4.1.1. Quantum reduction. The quantum analogue of symplectic induction discussed in section 2.1.1 is the ordinary induction. For completeness we recall the standard definitions (cf., for example, Dixmier (1974)). Let $\mathfrak{g}$ be a Lie algebra. Let us denote by Rep $\mathfrak{g}$ the category of $\mathcal{U}(\mathfrak{g})$-modules. Let $\mathfrak{b} \subset \mathfrak{g}$ be a Lie subalgebra. In complete analogy with section 2.2.1 we construct the induction functor $\operatorname{Ind}_{\mathfrak{b}}^{\mathfrak{g}}: \operatorname{Rep}_{\mathfrak{b}} \rightsquigarrow \operatorname{Rep}_{\mathfrak{g}}$ which associates to each $\mathcal{U}(\mathfrak{b})$-module an $\mathcal{U}(\mathfrak{g})$-module. Namely, we put $\operatorname{Ind} d_{\mathfrak{b}}^{\mathfrak{g}}(V)=\mathcal{U}(\mathfrak{g}) \boldsymbol{\bigotimes}_{\mathcal{U}(\mathfrak{b})} V$, where $\mathcal{U}(\mathfrak{g})$ is regarded as a right $\mathcal{U}(\mathfrak{b})$-module. [By definition, $\mathcal{U}(\mathfrak{g}) \bigotimes_{\mathcal{U}(\mathfrak{b})} V$ is the quotient of $\mathcal{U}(\mathfrak{g}) \bigotimes_{\mathbb{C}} V$ over its submodule generated by $(u \otimes v) \cdot X, u \in \mathcal{U}(\mathfrak{g}), v \in V, X \in \mathcal{U}(\mathfrak{b})$; the (right) action of $\mathcal{U}(\mathfrak{b})$ on $\mathcal{U}(\mathfrak{g}) \bigotimes_{\mathbb{C}} V$ is defined by

$$
(u \otimes v) \cdot X=\sum_{i} u S\left(X^{(i)}\right) \otimes X_{(i)} v
$$

where $\Delta X=\sum_{i} X^{(i)} \otimes X_{(i)}$ is the coproduct in $\mathcal{U}(\mathfrak{b})$ and $S$ is its antipode. In physical terms passing to the quotient means that we impose 'constraints on the wave functions'; these constraints express invariance of wave functions with respect to the diagonal action of $\mathcal{U}(\mathfrak{b})$.] The structure of a $\mathcal{U}(\mathfrak{g})$-module in $\operatorname{Ind} d_{\mathfrak{b}}^{\mathfrak{g}}(V)$ is induced by the left action of $\mathcal{U}(\mathfrak{g})$ on itself.

Fix a point $F \in \mathfrak{b}^{*}$ and assume that $\mathfrak{l}_{F}$ is a Lagrangian subalgebra subordinate to $F$. In that case $F$ defines a character of $\mathfrak{l}_{F}$; let $V_{F}$ be the corresponding 1-dimensional $\mathcal{U}\left(\mathfrak{l}_{F}\right)$-module. Put $V=\mathcal{U}(\mathfrak{b}) \bigotimes_{\mathcal{U}\left(\mathfrak{l}_{F}\right)} V_{F} . V$ is a natural $\mathcal{U}(\mathfrak{b})$-module associated with the coadjoint orbit of $F$. 
Proposition 4.3 $\operatorname{Ind}_{\mathfrak{b}}^{\mathfrak{g}}(V) \simeq \operatorname{Ind}_{\mathfrak{l}_{F}}^{\mathfrak{g}}\left(V_{F}\right)$.

Informally, we may say that an $\mathcal{U}(\mathfrak{g})$-module associated with a coadjoint orbit admitting a Lagrangian polarization may be induced from a 1-dimensional module.

Let us now return to the setting of theorem 4.1. Let $(\mathfrak{g}, r)$ be a Lie algebra equipped with a classical r-matrix $r \in$ End $\mathfrak{g}$ satisfying $(2.8)$. We regard $\mathcal{U}(\mathfrak{g})$ as a $\mathcal{U}\left(\mathfrak{g}_{r}\right)$-module with respect to the action (4.2). Let $V$ be a $\mathcal{U}\left(\mathfrak{g}_{r}\right)$-module.

Proposition $4.4 V \simeq \mathcal{U}(\mathfrak{g}) \bigotimes_{\mathcal{U}\left(\mathfrak{g}_{r}\right)} V$ as a linear space.

Since half of $\mathcal{U}\left(\mathfrak{g}_{r}\right)$ is acting on $\mathcal{U}(\mathfrak{g})$ on the left and the other half on the right, the structure of a $\mathcal{U}(\mathfrak{g})$-module in $\mathcal{U}(\mathfrak{g}) \bigotimes_{\mathcal{U}\left(\mathfrak{g}_{r}\right)} V$ is destroyed. However, the structure of $\mathcal{Z}$-module in $\mathcal{U}(\mathfrak{g})$ survives tensoring with $V$ over $\mathcal{U}\left(\mathfrak{g}_{r}\right)$.

Proposition 4.5 For any $\zeta \in \mathcal{Z}, u \in \mathcal{U}(\mathfrak{g}), v \in V, \zeta u \otimes v=u \otimes i_{r}(\zeta) v$.

This gives a new proof of theorem 4.1.

Fix $F \in \mathfrak{g}_{r}^{*}$ and let $\mathfrak{l}_{F} \subset \mathfrak{g}_{r}$ be a Lagrangian subalgebra subordinate to $F$. Let $V_{F}$ be the corresponding 1-dimensional $\mathcal{U}\left(\mathfrak{l}_{F}\right)$-module. Then $\mathcal{U}(\mathfrak{g}) \bigotimes_{\mathcal{U}\left(\mathfrak{l}_{F}\right)} V_{F}$ may be identified with $V$.

Let us assume now that $\mathfrak{g}=\mathfrak{g}_{+} \dot{+} \mathfrak{g}_{-}$and the r-matrix is given by (2.10); in that case $\mathcal{U}\left(\mathfrak{g}_{r}\right) \simeq \mathcal{U}\left(\mathfrak{g}_{+}\right) \otimes \mathcal{U}\left(\mathfrak{g}_{-}\right)$. Let $W$ be a $\mathcal{U}\left(\mathfrak{g}_{-}\right)$-module, $\mathbf{W}=\mathcal{U}(\mathfrak{g}) \bigotimes_{\mathcal{U}\left(\mathfrak{g}_{-}\right)} W$; we regard $\mathbf{W}$ as a left $\mathcal{U}(\mathfrak{g})$-module. There is a canonical embedding $W \hookrightarrow \mathbf{W}: w \mapsto 1 \otimes w$. Let $\mathbf{W}^{*}$ be the dual module regarded as a right $\mathcal{U}(\mathfrak{g})$-module, and let $W_{0}^{*} \subset \mathbf{W}$ be the subspace of $\mathcal{U}\left(\mathfrak{g}_{+}\right)$-invariants.

Lemma 4.6 $W_{0}^{*}$ is isomorphic to the dual of $W$.

This is an easy corollary of the decomposition

$$
\mathcal{U}(\mathfrak{g}) \simeq \mathcal{U}\left(\mathfrak{g}_{+}\right) \otimes \mathcal{U}\left(\mathfrak{g}_{-}\right) \simeq \mathfrak{g}_{+} \mathcal{U}(\mathfrak{g}) \oplus \mathcal{U}\left(\mathfrak{g}_{-}\right)
$$

Fix a basis $\left\{e_{i}\right\}$ in $W$, and let $\left\{e^{i}\right\}$ be the dual basis in $W_{0}^{*}$. Let $\Omega=\sum e^{i} \otimes e_{i} \in W_{0}^{*} \otimes W$ be the canonical element; it defines a natural mapping

$$
\mathbf{W} \rightarrow W: \varphi \mapsto\langle\varphi\rangle_{\Omega}=\sum_{i}\left\langle e^{i}, \varphi\right\rangle e_{i}
$$

Proposition 4.7 For any $\varsigma \in \mathcal{Z}, \varphi \in \mathbf{W},\langle\varsigma \varphi\rangle_{\Omega}=i_{\varsigma}\langle\varphi\rangle_{\Omega}$.

Informally, we may say that quantum Hamiltonians acting in $W$ are radial parts of the Casimir operators. (The reasons for this terminology will be obvious from our next example.) 
4.1.2. Toda lattice. Our first example is the generalized open Toda lattice. We retain the notation of section 2.2.1. Let again $\mathfrak{g}$ be a real split semisimple Lie algebra, $\mathfrak{g}=\mathfrak{k} \dot{+} \mathfrak{a} \dot{\mathfrak{n}}$ its Iwasawa decomposition, $\mathfrak{b}=\mathfrak{a} \dot{+} \mathfrak{n}$ the Borel subalgebra. Let $\mathfrak{d}_{p} \subset \mathfrak{b}(p \geq 0)$ be the generalized diagonals defined in (2.17). The subalgebras $\mathfrak{b}^{(p)}=\oplus_{r \geq p} \mathfrak{d}_{r}$ define a decreasing ad $\mathfrak{b}$-invariant filtration in $\mathfrak{b}$. Put $\mathfrak{p}_{k}=\oplus_{0 \leq p \leq k} s\left(\mathfrak{d}_{p}\right)$, where $s: \mathfrak{g} \rightarrow \mathfrak{p}: X \longmapsto \frac{1}{2}(i d-\sigma) X$ is the projection onto the subspace of anti-invariants of the Cartan involution $\sigma$. Put $\mathfrak{s}=\mathfrak{b} / \mathfrak{b}^{(2)}$. Let $L \in \mathfrak{p}_{1} \otimes \mathfrak{s} \subset$ $\mathfrak{p}_{1} \otimes \mathcal{U}(\mathfrak{s})$ be the canonical element induced by the natural pairing $\mathfrak{p}_{1} \times \mathfrak{b} / \mathfrak{b}^{(2)} \rightarrow \mathbb{R}$. Let $P: \mathcal{U}(\mathfrak{g}) \rightarrow \mathcal{U}(\mathfrak{b})$ be the projection map onto $\mathcal{U}(\mathfrak{b})$ in the decomposition $\mathcal{U}(\mathfrak{g})=\mathcal{U}(\mathfrak{b}) \oplus \mathcal{U}(\mathfrak{g}) \mathfrak{k}$, $p: \mathcal{U}(\mathfrak{b}) \rightarrow U(\mathfrak{s})$ the specialization map. Let us put $I_{\mathfrak{s}}=p \circ P(\mathcal{Z})$, where as usual $\mathcal{Z}$ is the center of $\mathcal{U}(\mathfrak{g})$. Fix a faithful representation $(\rho, V)$ of $\mathfrak{g}$ and put $L_{V}=(\rho \otimes i d) L$.

Proposition 4.8 (i) $I_{\mathfrak{s}}$ is a commutative subalgebra in $\mathcal{U}(\mathfrak{s})$; moreover, $\mathcal{Z} \rightarrow I_{\mathfrak{s}}: z \longmapsto H_{z}$ is an algebra isomorphism. (ii) $I_{\mathfrak{s}}$ is generated by $H_{k}=\operatorname{tr}_{V} L_{V}^{k} \in \mathcal{U}(\mathfrak{s}), k=1,2, \ldots$

Recall that $\mathfrak{s}=\mathfrak{a} \ltimes \mathfrak{u}$, where $\mathfrak{u}=\mathfrak{n} /[\mathfrak{n}, \mathfrak{n}]$, and the corresponding Lie group is $S=A \ltimes U$, $U=N / N^{\prime}$. Let $\mathcal{O}_{T}$ be the coadjoint orbit of $S$ described in (2.19), $f=\sum_{\alpha \in P}\left(e_{\alpha}+e_{-\alpha}\right) \in \mathcal{O}_{T}$ the marked point, $L_{f}=U$ the corresponding Lagrangian subgroup, $V_{f}=\mathbb{C}$ the 1-dimensional $U$-module which corresponds to $f$; we may also regard it as a $\mathcal{U}(\mathfrak{u})$-module.

Proposition 4.9 (i) The irreducible $\mathcal{U}(\mathfrak{s})$-module associated with $\mathcal{O}_{T}$ is the induced module $\mathcal{U}(\mathfrak{s}) \otimes_{\mathcal{U}(\mathfrak{u})} V_{f}$. (ii) The corresponding unitary representation space $\mathfrak{H}$ may be identified with $L_{2}(\mathfrak{a})$. (iii) Let $\Delta \in \mathcal{Z}$ be the quadratic Casimir operator which corresponds to the Killing form. Then $H_{\Delta}$ is precisely the Toda Hamiltonian $H_{T}$ acting in $\mathfrak{H}$; it is given by

$$
H_{T}=-(\partial, \partial)+\sum_{\alpha \in P} \exp 2 \alpha(q), \partial=\left(\frac{\partial}{\partial q_{1}}, \ldots, \frac{\partial}{\partial q_{n}}\right) .
$$

In other words, geometric quantization of the Toda Hamiltonian agrees with its 'naive' Schroedinger quantization. What is nontrivial, of course, is the consistent definition of the quantum integrals of motion which correspond to higher Casimirs.

Let us now turn to the description of the Toda lattice based on the reduction procedure. In section 4.1.1 we discussed the reduction using the language of $\mathcal{U}(\mathfrak{g})$-modules; in the present setting we may assume that all these modules are actually integrable, i.e., come from representations of the corresponding Lie groups. In particular, the action of $\mathcal{U}(\mathfrak{g})$ on itself by left (right) multiplications corresponds to the regular representation of $G$; we may regard $\mathfrak{H}=L_{2}(G)$ as a result of quantization of $T^{*} G$.

We are led to the following construction. Let $\chi_{f}$ be the character of $N$ defined by

$$
\chi_{f}(\exp X)=\exp \text { if }(X), X \in \mathfrak{n} .
$$

Let $\mathfrak{H}_{f}$ be the space of smooth functions on $G$ satisfying the functional equation

$$
\psi(k x n)=\chi_{f}(n) \psi(x), k \in K, n \in N
$$

By Iwasawa decomposition, any such function is uniquely determined by its restriction to $A \subset G$. Thus there is an isomorphism $i: C^{\infty}(\mathfrak{a}) \rightarrow \mathfrak{H}_{f}: i(\psi)(k a n)=\chi_{f}(n) \psi(\log a)$. 
Lemma 4.10 $\mathfrak{H}_{f}$ is invariant with respect to the action of $\mathcal{Z} \subset \mathcal{U}(\mathfrak{g})$.

For $\zeta \in \mathcal{Z}$ let $\delta(\zeta) \in E n d C^{\infty}(\mathfrak{a})$ be its radial part defined by

$$
\zeta i(\psi)=i(\delta(\zeta) \psi), \psi \in C^{\infty}(\mathfrak{a}) .
$$

Proposition 4.11 We have $\delta(\zeta)=H_{\zeta}$, i.e., the radial parts of the Casimir operators coincide with the quantum integrals of the Toda lattice.

We are interested in eigenfunctions of the Toda Hamiltonian and higher Toda integrals. By proposition 4.11 this is equivalent to description of the eigenfunctions of Casimir operators on $G$ which satisfy the functional equation (4.3). At the formal level this problem may be solved as follows. Let $(\pi, \mathfrak{V})$ be an infinitesimally irreducible representation of $G, \mathfrak{V}^{*}$, the dual representation. Assume that $w \in \mathfrak{V}$ satisfies

$$
\pi(n) w=\chi_{f}(n) w \text { for } n \in N .
$$

(In that case $w$ is called a Whittaker vector.) Assume, moreover, that in the dual space $\mathfrak{V}^{*}$ there is a $K$-invariant vector $v \in \mathfrak{V}^{*}$. Put

$$
\psi(x)=\langle v, \pi(x) w\rangle .
$$

Proposition $4.12 \psi$ is an eigenfunction of $\mathcal{Z}$ and satisfies the functional equation (4.3).

Thus $\psi$ is essentially a Toda lattice eigenfunction.

This formal argument can be made rigorous. Since the Toda Hamiltonian describes scattering in a repulsive potential, its spectrum is continuous; so there are no chances that $\psi$ (which is called a Whittaker function) is in $L_{2}$. The problem is to find representations of $G$ such that $\psi$ has the usual properties of a continuous spectrum eigenfunction (i.e., the wave packets smoothed down with appropriate amplitudes are in $L_{2}$ ). As it appears, the correct class are spherical principal series representations.

Definition 4.1 Let $B=M A N$ be the Borel subgroup in $G, \lambda \in \mathfrak{a}^{*}$; let $\chi_{\lambda}: B \rightarrow \mathbb{C}$ be the 1-dimensional representation defined by

$$
\chi_{\lambda}(\operatorname{man})=\exp \langle\lambda-\rho, \log a\rangle .
$$

The spherical principal series $\pi_{\lambda}$ is the induced representation, $\pi_{\lambda}=i n d_{B}^{G} \chi_{\lambda}$.

Principal series representations are infinitesimally irreducible and hence define a homomorphism $\pi: \mathcal{Z} \rightarrow P\left(\mathfrak{a}^{*}\right)$ into the algebra of polynomials on $\mathfrak{a}^{*}$

$$
\pi: z \longmapsto \pi_{z} \text {, where } \pi_{\lambda}(z):=\pi_{z}(\lambda) \cdot i d .
$$

By a classical Harish Chandra theorem (Dixmier (1974)) $\pi$ is actually an isomorphism onto the subalgebra $P\left(\mathfrak{a}^{*}\right)^{W} \subset P\left(\mathfrak{a}^{*}\right)$ of the Weyl group invariants. Hence the set of principal series representations is sufficiently ample to separate points of the algebraic spectrum of $\mathcal{Z}$. 
The algebraic theory of Whittaker vectors is exposed in Kostant (1978), the analytic theory leading to the Plancherel theorem for the Toda lattice is outlined in Semenov-Tian-Shansky (1994a). A remarkable point is that the eigenfunctions of the quantum system are expressed as matrix coefficients of appropriate representations of the 'hidden symmetry group'. This is a special case of a very general situation. Below we shall discuss another example of this kind, for which the hidden symmetry algebra is infinite-dimensional.

4.1.2. Gaudin model. The treatment of the Gaudin model is considerably more difficult, since in this case the hidden symmetry group is infinite-dimensional. Remarkably, the general pattern described in section 4.1.1 is fully preserved in this case as well.

The algebra of observables for the Gaudin model is simply $\mathcal{U}\left(\mathfrak{g}^{N}\right)$. Let $V_{\lambda}$ be a finitedimensional highest weight representation of $\mathfrak{g}$ with dominant integral highest weight $\lambda$. Let $\lambda=\left(\lambda_{1}, \ldots \lambda_{N}\right)$ be the set of such weights; put $\mathbf{V}_{\lambda}=\otimes_{i} V_{\lambda_{i}}$. The space $\mathbf{V}_{\lambda}$ is a natural Hilbert space associated with the Gaudin model; so the 'kinematical' part of quantization problem in this case is fairly simple. Let us fix also an auxiliary representation $(\rho, V)$. By analogy with the classical case, we may introduce the quantum Lax operator,

$$
L_{V}(z) \in \operatorname{EndV}(z) \otimes \mathfrak{g}^{N} \subset \operatorname{EndV}(z) \otimes \mathcal{U}\left(\mathfrak{g}^{N}\right) ;
$$

the definition remains exactly the same, but now we embed $\mathfrak{g}^{N}$ into the universal enveloping algebra $\mathcal{U}\left(\mathfrak{g}^{N}\right)$ instead of the symmetric algebra $\mathcal{S}\left(\mathfrak{g}^{N}\right)$. The commutation relations for $L_{V}(z)$ essentially reproduce the Poisson brackets relations (2.28), but this time the l.h.s. is a matrix of true commutators:

$$
\left[L_{V}(u) \otimes, L_{V}(v)\right]=\left[r_{V}(u, v), L_{V}(u) \otimes 1+1 \otimes L_{V}(v)\right] .
$$

The key point in (4.7) is the interplay of commutation relations in the quantum algebra $\mathcal{U}\left(\mathfrak{g}^{N}\right)$ and the auxiliary matrix algebra $\operatorname{EndV}(z)$. Formula (4.7) was the starting point of QISM (as applied to models with linear commutation relations). Put

$$
S(u)=\frac{1}{2} \operatorname{tr}_{V}\left(L_{V}(u)\right)^{2} ;
$$

using (4.7), it is easy to check that $S(u)$ form a commutative family of Hamiltonians (called Gaudin Hamiltonians) in $\mathcal{U}\left(\mathfrak{g}^{N}\right)$ (see, e.g., Jurčo (1989) ). An important property of this commuting family is that it possesses at least one 'obvious' eigenvector $\mid 0>\in H$, the tensor product of highest weight vectors in $V_{\lambda_{i}}$; it is usually called the vacuum vector .

One of the key ideas of QISM is to construct other eigenvectors by applying to the vacuum creation operators which are themselves rational functions of $z$. This construction is calledthe algebraic Bethe Ansatz. Assume that $\mathfrak{g}=\mathfrak{s l}_{2}$ and let $\{E, F, H\}$ be its standard basis. Put

$$
F(z)=\sum_{z_{i} \in D} \frac{F^{(i)}}{z-z_{i}},
$$


where $F^{(i)}$ acts as $F$ in the i-th copy of $\mathfrak{s l}_{2}$ and as $i d$ in other places. The Bethe vector is, by definition,

$$
\left|w_{1}, w_{2}, \ldots w_{m}>=F\left(w_{1}\right) F\left(w_{2}\right) \ldots F\left(w_{m}\right)\right| 0>.
$$

The Lax matrix (4.6) applied to $\mid w_{1}, w_{2}, \ldots w_{m}>$ becomes triangular, i.e.,

$$
L(u) \mid w_{1}, w_{2}, \ldots w_{m}>=\left(\begin{array}{cc}
a\left(u, w_{1}, w_{2}, \ldots w_{m}\right) & * \\
0 & d\left(u, w_{1}, w_{2}, \ldots w_{m}\right)
\end{array}\right)
$$

after a short computation this yields

$$
\begin{aligned}
& S(u) \mid w_{1}, w_{2}, \ldots w_{m}>= \\
& \quad s_{m}(u)\left|w_{1}, w_{2}, \ldots w_{m}>+\sum_{j=1}^{N} \frac{f_{j}}{u-w_{j}}\right| w_{1}, w_{2}, \ldots, w_{j-1}, u, w_{j+1}, \ldots, w_{m}>,
\end{aligned}
$$

where

$$
f_{j}=\sum_{i=1}^{N} \frac{\lambda_{i}}{w_{j}-z_{i}}-\sum_{s \neq j} \frac{2}{w_{j}-w_{s}}
$$

and $s_{m}(u)$ is a rational function,

$$
\begin{gathered}
s_{m}(u)=\frac{c_{V}}{2} \chi_{m}(u)^{2}-c_{V} \partial_{u} \chi_{m}(u), \\
\chi_{m}(u)=\sum_{i=1}^{N} \frac{\lambda_{i}}{u-z_{i}}-\sum_{i=1}^{m} \frac{2}{u-w_{j}} .
\end{gathered}
$$

(The constant $c_{V}$ depends on the choice of $V$.) If all $f_{j}$ vanish, $\mid w_{1}, w_{2}, \ldots w_{m}>$ is an eigenvector of $S(u)$ with the eigenvalue $s_{m}(u)$; equations

$$
\sum_{i=1}^{N} \frac{\lambda_{i}}{w_{j}-z_{i}}-\sum_{s \neq j} \frac{2}{w_{j}-w_{s}}=0
$$

are called the Bethe Ansatz equations. (Notice that (4.12) is precisely the condition that $s_{m}(u)$ be nonsingular at $u=w_{i}$.)

For general simple Lie algebras the study of spectra of the Gaudin Hamiltonians becomes rather complicated; one way to solve this problem is to treat it inductively by choosing in $\mathfrak{g}$ a sequence of embedded Lie subalgebras of lower rank and applying the algebraic Bethe Ansatz to these subalgebras. An alternative idea (which is completely parallel to the treatment of the Toda lattice in section 4.1) is to interpret the Hamiltonians as radial parts of (infinite dimensional) Casimir operator of the 'global' Lie algebra $\mathfrak{g}_{D}$.

It is impossible to apply theorem 4.1 immediately in the affine case, since the center of $\mathcal{U}\left(\mathfrak{g}_{D}\right)$ is trivial. The point is that the invariants in the (suitably completed) symmetric algebra $\mathcal{S}\left(\mathfrak{g}_{D}\right)$ are infinite series; an attempt to quantize these expression leads to divergent expressions, unless some kind of ordering prescription is introduced. The commutation relations for the normally ordered operators are already nontrivial and they do not lie in the center of $\mathcal{U}\left(\mathfrak{g}_{D}\right)$. However, the situation can be amended by first passing to the central extension of $\mathcal{U}\left(\mathfrak{g}_{D}\right)$ and then considering the quotient algebra $\mathcal{U}_{k}\left(\mathfrak{g}_{D}\right)=U\left(\hat{\mathfrak{g}}_{D}\right) /(c-k)$. It is known that for the critical value of the 
central charge $k=-h^{\vee}$ (here $h^{\vee}$ is the dual Coxeter number of $\mathfrak{g}$ ) the (appropriately completed) algebra $\mathcal{U}_{-h^{\vee}}\left(\mathfrak{g}_{D}\right)$ possesses an ample center.

Let us recall first of all the construction of Sugawara operators. Let $L \mathfrak{g}=\mathfrak{g}((z))$ be the 'local' algebra of formal Laurent series with coefficients in $\mathfrak{g}$,Let $\omega$ be the 2-cocycle on $L \mathfrak{g}$ defined by

$$
\omega(X, Y)=\operatorname{Res}_{z=0}\left\langle X, \partial_{z} Y\right\rangle d z
$$

It is well known that highest weight representations of $L \mathfrak{g}$ are actually projective and correspond to the central extension $\widehat{L \mathfrak{g}} \simeq L \mathfrak{g} \oplus \mathbb{C} c$ of $L \mathfrak{g}$ defined by this cocycle. Let us consider formally the canonical element $J \in L \mathfrak{g} \otimes L \mathfrak{g}$ which corresponds to the inner product

$$
(X, Y)=\operatorname{Res}_{z=0}\langle X, Y\rangle d z
$$

in $L \mathfrak{g}$. Fix a finite-dimensional representation $(\rho, V)$ of $\mathfrak{g}$; it extends to the 'evaluation representation' of $L \mathfrak{g}$ in $V \otimes_{\mathbb{C}} \mathbb{C}((z))$. Let $\left(\pi_{k}, \mathfrak{V}\right)$ be any level $k$ highest weight representation of $\widehat{L \mathfrak{g}}$. (This means that the central element $c \in \widehat{L \mathfrak{g}}$ acts by $\pi_{k}(c)=k \cdot i d$.) Put $J(z)=\left(\pi_{k} \otimes \rho\right) J$. Since $J$ is not a proper element in $L \mathfrak{g} \otimes L \mathfrak{g}, J(z)$ is a formal series infinite in both directions; however, its coefficients are well defined. Namely, let $\left\{e_{a}\right\}$ be a basis in $\mathfrak{g}$; the algebra $L \mathfrak{g}$ is spanned by the vectors $e_{a}(n)=e_{a} \otimes z^{n}, n \in \mathbb{Z}$. It is easy to see that the coefficients of $J(z)$ are finite linear combinations of the vectors $\pi_{k}\left(e_{a}(n)\right.$.)

Put

$$
T(u)=\frac{1}{2}: \operatorname{tr} J(u)^{2}:, T(u)=\sum_{n=-\infty}^{\infty} T_{n} u^{n}
$$

where the normal ordering :: is defined in the following way:

$$
\begin{aligned}
& : e_{a}(n) e_{b}(m):=e_{b}(m) e_{a}(n), \text { if } n<0, m \geq 0, \\
& : e_{a}(n) e_{b}(m):=e_{a}(n) e_{b}(m) \text { otherwise. }
\end{aligned}
$$

Due to normal ordering the coefficients of $T(u)$ are well defined operators in EndVI. It is well known that

$$
\begin{aligned}
& {\left[T_{m}, T_{n}\right]=\left(k+h^{\vee}\right)\left[(m-n) T_{m+n}+\frac{k \operatorname{dim} \mathfrak{g}}{12}\left(n^{3}-n\right) \delta_{n,-m}\right],} \\
& {\left[T_{m}, \pi_{k}\left(e_{a}(n)\right)\right]=-\left(k+h^{\vee}\right) \pi_{k}\left(e_{a}(n+m)\right) .}
\end{aligned}
$$

Therefore, if $k \neq-h^{\vee}, T_{n}$ generate the Virasoro algebra; for $k=-h^{\vee}$ the elements $T_{n}, n \in \mathbb{Z}$, are central.

One can show that for $\mathfrak{g}=\mathfrak{s l}_{2}$ the center of $\mathcal{U}_{-h^{\vee}}(\widehat{L \mathfrak{g}})$ is generated by $T_{n}, n \in \mathbb{Z}$; f for higher rank algebras there are other Casimirs. An attempt to construct these higher Casimir elements by considering : $\operatorname{tr} J(u)^{n}$ : for arbitrary $n \geq 2$ runs into trouble. However, by applying a different technique Feigin and Frenkel (1992) have proved that for the critical central charge the algebra of Casimirs is very ample: essentially, there exists a Casimir element with prescribed symbol and hence there is an isomorphism $\mathcal{S}(L \mathfrak{g})^{L \mathfrak{g}} \rightarrow \mathcal{Z}\left(U_{-h^{\vee}}(\widehat{L \mathfrak{g}})\right)$.

The situation with the Lie algebra $\mathfrak{g}_{D}$ is basically the same as described above. This allows to realize the Gaudin Hamiltonians as radial parts of appropriate Casimir operators. Let us 
describe this construction (due to Feigin, Frenkel and Reshetikhin (1994)) more precisely. Let the Lie algebras $\mathfrak{g}_{D}, \mathfrak{g}_{D}^{+}, \mathfrak{g}_{D}^{++}, \mathfrak{g}(D)$ be as above. It will be convenient to add one more point $\{u\}$ to the divisor $D$ and to attach to it the trivial representation $V_{0}$ of $\mathfrak{g}$. (This will not affect the Hilbert space of our model, since $\otimes_{z_{i} \in D} V_{\lambda_{i}} \otimes V_{0}=\left(\otimes_{z_{i} \in D} V_{\lambda_{i}}\right) \otimes \mathbb{C} \simeq \otimes_{z_{i} \in D} V_{\lambda_{i}}$.) Thus we write $D^{\prime}=D \cup\{u\}$, etc. Let $\omega$ be the 2-cocycle on $\mathfrak{g}_{D^{\prime}}$ defined by

$$
\omega(X, Y)=\sum_{z_{i} \in D^{\prime}} \operatorname{Res}\left(X_{i}, d Y_{i}\right)
$$

Let $\hat{\mathfrak{g}}_{D^{\prime}}=\mathfrak{g}_{D^{\prime}} \oplus \mathbb{C} c$ be the central extension of $\mathfrak{g}_{D^{\prime}}$ defined by this cocycle. Note that since the restriction of $\omega$ to the subalgebra $\mathfrak{g}\left(D^{\prime}\right) \subset \mathfrak{g}_{D^{\prime}}$ is zero, the algebra $\mathfrak{g}\left(D^{\prime}\right)$ is canonically embedded into $\hat{\mathfrak{g}}_{D^{\prime}}$. Put $\hat{\mathfrak{g}}_{D^{\prime}}^{+}=\mathfrak{g}_{D^{\prime}}^{+} \oplus \mathbb{C} c$. Fix a highest weight representation $V_{(\lambda, 0)}=\otimes_{z_{i} \in D} V_{\lambda_{i}} \otimes V_{0}$ of the Lie algebra $\mathfrak{g}^{N+1}=\mathfrak{g}_{D^{\prime}}^{+} / \mathfrak{g}_{D^{\prime}}^{++}$as above and let $V_{(\lambda, 0)}^{k}$ be the associated representation of $\hat{\mathfrak{g}}_{D^{\prime}}^{+}$on which the center $\mathbf{C} c$ acts by multiplication by $k \in \mathbb{Z}$.. Let $\mathbf{V}_{(\lambda, 0)}^{k}$ be the induced representation of $\hat{\mathfrak{g}}_{D^{\prime}}$,

$$
\mathbf{V}_{(\lambda, 0)}^{k}=\mathcal{U}\left(\hat{\mathfrak{g}}_{D}\right) \otimes_{\mathcal{U}\left(\hat{\mathfrak{g}}_{D^{\prime}}^{+}\right)} V_{(\lambda, 0)}^{k}
$$

There is a canonical embedding $V_{(\lambda, 0)}^{k} \hookrightarrow \mathbf{V}_{(\lambda, 0)}^{k}: v \mapsto 1 \otimes v$. Let $\left(\mathbf{V}_{(\lambda, 0)}^{k}\right)^{*}$ be the dual of $\mathbf{V}_{(\lambda, 0)}$, $\mathbf{H}_{(\lambda, 0)}^{k} \subset\left(\mathbf{V}_{(\lambda, 0)}^{k}\right)^{*}$ the subspace of $\mathfrak{g}\left(D^{\prime}\right)$-invariants. Decomposition (2.24) together with the obvious isomorphism $V_{(\lambda, 0)} \simeq V_{\lambda}$ immediately implies that $\mathbf{H}_{(\lambda, 0)}$ is canonically isomorphic to $V_{\lambda}^{*}$. Let $\Omega \in \mathbf{H}_{\lambda} \otimes V_{\lambda}$ be the canonical element; it defines a natural mapping $\mathbf{V}_{(\lambda, 0)}^{k,} \rightarrow$ $V_{\lambda}: \varphi \mapsto\langle\varphi\rangle_{\Omega}$. (In Conformal Field Theory $\langle\varphi\rangle_{\Omega}$ are usually called correlation functions.) For any $x \in U\left(\hat{\mathfrak{g}}_{D}\right)$ let $\gamma_{\lambda}(x) \in E n d V_{\lambda}$ be the linear operator defined by the composition mapping $v \mapsto\langle x(1 \otimes v)\rangle_{\Omega}, v \in V_{\lambda}$.

Now suppose that $k=-h^{\vee}$; let again $J$ be the canonical element which corresponds to the inner product $(2.25)$ in $\mathfrak{g}((z)), J(z)=(i d \otimes \rho) T, T(z)=t r: J(z)^{2}$ : Let us embed $\mathcal{U}_{-h^{\vee}}(\mathfrak{g}((z)))$ into $\mathcal{U}_{-h^{\vee}}\left(\mathfrak{g}_{D}\right)$ sending it to the extra place $\{u\} \subset D^{\prime}$ to which we attached the trivial representation of $\mathfrak{g}$.

Proposition 4.13 $S(u)=\gamma_{\lambda}\left(J_{V}(-2)\right)$ coincides with the Gaudin Hamiltonian.

Remark 4.1 Besides quadratic Hamiltonians associated with the Sugawara current there are also higher commuting Hamiltonians which may be obtained using the methods of Feigin and Frenkel (1992).

In the calculation above we started with a generalized Verma module $\mathbf{V}_{(\lambda, 0)}^{k}$; however, any representation of the critical level will do. The problem is to find a sufficiently ample class of critical level representations which will account for the spectrum of the Gaudin model. Note that this construction is exactly similar to the description of the Toda eigenfunctions in the finite-dimensional case. In the Toda case the correct class consisted of the spherical principal series representations; an important point is that principal series representations separate points of the algebraic spectrum of $\mathcal{Z}$. Now, at the critical level the algebra of the Casimir operators 
is extraordinary rich; hence generalized Verma modules which are parametrized by the dual of the extended Cartan subalgebra $\widehat{\mathfrak{a}}=\mathfrak{a} \oplus \mathbb{C}$ (i.e. depend on a finite number of parameters) can not be used. Remarkably, there is another class of representations of $\mathfrak{g}_{D}$, the Wakimoto modules, which play the role of the principal series representations. Roughly, the idea is to use the loop algebra $L \mathfrak{a}=\mathfrak{a} \otimes \mathbb{C}((z))$ as the substitute of the Cartan subalgebra, to extend its characters trivially to $L \mathfrak{n}$ and to take the induced $L \mathfrak{g}$-module. Due to normal ordering (which is necessary, since after central extension $L \mathfrak{a}$ becomes a Heisenberg algebra), this construction should be modified (among other things, this leads to a shift of the level, i.e., of the value of the central charge, which becomes $-h^{\vee}$ ).

For $\mathfrak{g}=\mathfrak{s l}_{2}$ the Wakimoto module of the critical level has the following explicit realization (Frenkel (1995)). Let $\mathrm{H}$ be the Heisenberg algebra with generators $a_{n}, a_{n}^{*}, n \in \mathbb{Z}$, and relations

$$
\left[a_{n}, a_{m}^{*}\right]=\delta_{n,-m}
$$

Let $\mathfrak{F}$ be the Fock representation of $\mathrm{H}$ with vacuum vector $v$ satisfying $a_{n} v=0, n \geq 0, a_{n}^{*} v=$ $0, n>0$. Put

$$
a(u)=\sum_{n \in \mathbb{Z}} a_{n} u^{-n-1}, a^{*}(u)=\sum_{n \in \mathbb{Z}} a_{n}^{*} u^{-n} .
$$

Let $\{E, F, H\}$ be the standard basis of $\mathfrak{s l}_{2}$. Put $E(n)=E \otimes z^{n}, F(n)=F \otimes z^{n}, H(n)=H \otimes z^{n}$; let

$$
E[u]=\sum_{n \in \mathbb{Z}} E(n) u^{n}, F[u]=\sum_{n \in \mathbb{Z}} F(n) u^{n}, H[u]=\sum_{n \in \mathbb{Z}} F(n) u^{n}
$$

be the corresponding 'generating functions'. For any formal power series $\chi(u)=\sum_{n \in \mathbb{Z}} \chi_{n} u^{-n-1}$ define the (projective) action of $L \mathfrak{g}$ on $F$ by

$$
\begin{aligned}
& E[u]=a(u), H[u]=-2: a(u) a^{*}(u):+\chi(u), \\
& F[u]=-: a(u) a^{*}(u) a^{*}(u):-2 \partial_{u} a^{*}(u)+\chi(u) a^{*}(u) .
\end{aligned}
$$

Thus we get an $L \mathfrak{g}$-module structure in $\mathfrak{F}$ which depends on $\chi(u)$; this is the Wakimoto module of the critical level $k=-2$ (usually denoted by $W_{\chi(u)}$ ).

The Virasoro generators $T_{n}, n \in \mathbb{Z}$, are scalar in $W_{\chi(u)}, T_{n}=q_{n} \cdot i d$. Put $q(u)=\sum_{n \in \mathbb{Z}} q_{n} u^{n}$. One can show that

$$
q(u)=\frac{1}{4} \chi(u)^{2}-\frac{1}{2} \partial_{u} \chi(u)
$$

i.e., $q$ is the Miura transform of $\chi$. This means of course that

$$
\partial_{u}^{2}-q(u)=\left(\partial_{u}-\frac{1}{2} \partial_{u} \chi\right)\left(\partial_{u}+\frac{1}{2} \partial_{u} \chi\right)
$$

as a matter of fact, due to normal ordering, if we make a change of coordinates on the (formal) disk, $q$ transforms as a projective connection. Now a comparison with formula (4.11) suggests 
that the Bethe Ansatz is related to a Wakimoto module with an appropriate choice of $\chi$. Namely, we consider the following global rational function

$$
\lambda(u)=\sum_{i=1}^{N} \frac{\lambda_{i}}{u-z_{i}}-\sum_{j=1}^{m} \frac{2}{u-w_{j}} .
$$

Denote by $\lambda_{i}\left(u-z_{i}\right)$ its expansion at the points $z_{i}, i=1, \ldots, N$, and by $\mu_{j}\left(u-w_{j}\right)$ its expansion at the points $w_{j}, j=1, \ldots, m$. We have

$$
\lambda_{i}(t)=\frac{\lambda_{i}}{t}+\ldots, \mu_{j}(t)=-\frac{2}{t}+\mu_{j}(0)+\ldots
$$

where

$$
\mu_{j}(0)=\sum_{i=1}^{N} \frac{\lambda_{i}}{w_{j}-z_{i}}-\sum_{s \neq j} \frac{2}{w_{j}-w_{s}} .
$$

We want to attach the Wakimoto modules to the points on the Riemann sphere. To this end let us observe that creation and annihilation operators (4.18) define a projective representation of the 'local' algebra $\Gamma=\mathbb{C}((u)) \oplus \mathbb{C}((u)) d u$ consisting of formal series and formal differentials with the cocycle

$$
\Omega_{0}\left(\left(\varphi_{1}, \alpha_{1}\right),\left(\varphi_{2}, \alpha_{2}\right)\right)=\operatorname{Res}_{u=0}\left(\varphi_{1} \alpha_{2}-\varphi_{2} \alpha_{1}\right) .
$$

'Global' algebra is the direct sum of local algebras with the cocycle

$$
\Omega\left(\left(\varphi_{1}, \alpha_{1}\right),\left(\varphi_{2}, \alpha_{2}\right)\right)=\sum_{i} \operatorname{Res}_{z_{i}}\left(\varphi_{1} \alpha_{2}-\varphi_{2} \alpha_{1}\right) .
$$

Now let us consider the tensor product of the Wakimoto modules attached to the points $z_{i}, w_{j}$,

$$
\mathbb{W}=\bigotimes_{i=1}^{N} W_{\lambda_{i}(t)} \bigotimes_{j=1}^{m} W_{\mu_{j}(t)}
$$

Let $\mathbb{H}$ be the corresponding 'big' Heisenberg algebra. The eigenfunctions of the Gaudin Hamiltonians may be constructed as the appropriate correlation functions associated with $\mathbb{W}$. To formulate the exact statement we need one more definition. Let $V$ be a $\mathfrak{g}((t))$-module. For $X \in \mathfrak{g}, n \in \mathbb{Z}$ we put $X(n)=X \otimes t^{n} \in \mathfrak{g}((t))$. A vector $v \in V$ is called a singular vector of imaginary weight if $X(n) v=0$ for all $X \in \mathfrak{g}, n \in \mathbb{Z}, n \geq 0$. Let $v_{j} \in W_{\mu_{j}(t)}$ be the vacuum vector; put $w_{j}=a_{-1} v_{j}$, where $a_{-1}$ is the creation operator introduced in (4.17).

Lemma 4.14. The vector $w_{j}$ is a singular vector of imaginary weight if and only if $\mu_{j}(0)=0$.

Note that this condition coincides with (4.12).

Let $\tilde{M}_{\lambda_{i}}$ be the subspace of $W_{\lambda_{i}(t)} \simeq \mathcal{F}$ generated from the vacuum vector by the creation operators $a_{0}^{*}$. 
Lemma 4.15 $\tilde{M}_{\lambda_{i}}$ is stable with respect to the subalgebra $\mathfrak{g} \subset L \mathfrak{g}$ of constant loops and is isomorphic to the dual of the Verma module $M_{\lambda_{i}}$ over $\mathfrak{g}=\mathfrak{s l}_{2}$ with the highest weight $\lambda_{i}=$ $\operatorname{Res}_{t=0} \lambda_{i}(t)$.

This assertion is immediate from the definition (4.19) of the $\widehat{\mathfrak{s l}_{2}}$-action on $\mathcal{F}$.

Let $\mathbb{W}^{*}$ be the dual of $\mathbb{W}$. . Let $\mathfrak{h}_{z, w}$ be the algebra of rational functions with values in the Cartan subalgebra $\mathfrak{h} \subset \mathfrak{s l}_{2}$ which are regular outside $\left\{z_{1}, \ldots, z_{N}, w_{1}, \ldots, w_{m}\right\}$. There is a natural embedding of $\mathfrak{h}_{z, w}$ into the 'big' Heisenberg algebra $H$ defined via expansion at each point of the divisor $\left\{z_{1}, \ldots, z_{N}, w_{1}, \ldots, w_{m}\right\}$. Let $\mathcal{H}_{z, w}$ be the maximal abelian subalgebra of $\mathbb{H}$ which contains $\mathcal{H}_{z, w}$.

Lemma 4.16 The space of $\mathcal{H}_{z, w}$-invariants in $\mathbb{W}^{*}$ is 1-dimensional; it is generated by a functional $\tau$ whose value on the tensor product of the vacuum vectors of $W_{\lambda_{i}(t)}, W_{\mu_{j}(t)}$ is equal to 1; the restriction of $\tau$ to $\otimes_{i=1}^{N} \tilde{M}_{\lambda_{i}} \otimes w_{1} \otimes \ldots \otimes w_{m}$ is nontrivial.

Let $\psi \in \otimes_{i=1}^{N} \tilde{M}_{\lambda_{i}}$. We may write $\tau(\psi)=\langle\psi, \phi\rangle$ where $\phi \in \otimes_{i=1}^{N} M_{\lambda_{i}}$ is a vector in the tensor product of Verma modules over $\mathfrak{s l}_{2}$.

Theorem 4.17 (Feigin, Frenkel and Reshetikhin (1994)) If the Bethe equations (4.12) are satisfied, $\phi \in \otimes_{i=1}^{N} M_{\lambda_{i}}$ is an eigenvector of the Gaudin Hamiltonians in $\otimes_{i=1}^{N} M_{\lambda_{i}}$ with the eigenvalue 4.11.

Remark 4.2 If the weights $\lambda_{i}$ are dominant integral, there is a natural projection

$$
\pi: \otimes_{i=1}^{N} M_{\lambda_{i}} \rightarrow \otimes_{i=1}^{N} V_{\lambda_{i}} .
$$

It is easy to see that $\pi$ maps the eigenvectors of the Gaudin Hamiltonians in $\otimes_{i=1}^{N} M_{\lambda_{i}}$ onto those in $\otimes_{i=1}^{N} V_{\lambda_{i}}$.

The construction described above admits a generalization to arbitrary semisimple Lie algebras. We shall only write down the generalized Bethe equations. To parametrize a Bethe vector first choose a set $\left\{w_{1}, \ldots, w_{m}\right\}, w_{j} \in \mathbf{C}$, and assign to each $w_{j}$ a set of simple roots $\left\{\alpha_{i_{j}}\right\}_{i=1}^{N}$, one for each copy of $\mathfrak{g}$ in $\mathfrak{g}^{N}$. Let $F_{i_{j}}^{i}$ be the corresponding Chevalley generator of $\mathfrak{g}$ acting nontrivially in the i-th copy of $\mathfrak{g}$. The straightforward generalization of the Bethe creation operator (4.9) to the higher rank case is

$$
F\left(w_{j}\right)=\sum_{i=1}^{N} \frac{F_{i_{j}}^{i}}{w_{j}-z_{i}}
$$

(Babujian and Flume (1993)). The problem with (4.21) is that these operators no longer commute with each other. Hence one cannot use a string of creation operators to produce an eigenvector as it is done in (4.10). The correct way to decouple them is to use the Wakimoto modules $W_{\mu_{j}(t)}$ which correspond to the poles of the creation operator; as above, the eigenvectors are generated by the singular vectors of imaginary weight which exist if and only if the 
constant term in the expansion of $\mu_{j}(t)$ satisfies certain orthogonality conditions. This leads to the generalized Bethe equations:

$$
\sum_{i=1}^{N} \frac{\left(\lambda_{i}, \alpha_{i_{j}}\right)}{w_{j}-z_{i}}-\sum_{s \neq j} \frac{\left(\alpha_{i_{s}}, \alpha_{i_{j}}\right)}{w_{j}-w_{s}}=0, j=1, \ldots, m .
$$

Bethe vectors are again computed as correlation functions.

Let us end this section with a brief remark on the Knizhnik-Zamolodchikov equations. Recall that this is a system of equations satisfied by the correlation functions for an arbitrary value of the central charge; the critical value $c=-h^{\vee}$ corresponds to the semiclassical limit for the KZ system (small parameter before the derivatives). The outcome of this is two-fold: first, the Bethe vectors for the Gaudin model appear naturally in the semiclassical asymptotics of the solutions of the KZ system (Reshetikhin and Varchenko (1993)). Moreover, the exact integral representation of the solutions (for any value of the central charge) also involves the Bethe vectors (Feigin, Frenkel and Reshetikhin (1994)).

\subsection{Quadratic case. Quasitriangular Hopf Algebras}

For an expert in Quantum Integrability the Gaudin model is certainly a sort of a limiting special case. The real thing starts with the quantization of quadratic Poisson bracket relations (3.7). This is a much more complicated problem which eventually requires the whole machinery of Quantum Groups (and has led to their discovery). The substitute for the Poisson bracket relations (3.30) is the famous relation

$$
R\left(u v^{-1}\right) L^{1}(u) L^{2}(v) R\left(u v^{-1}\right)^{-1}=L^{2}(v) L^{1}(u),
$$

where $R(u)$ is the quantum R-matrix satisfying the quantum Yang-Baxter identity

$$
R_{12}(u) R_{13}(u v) R_{23}(v)=R_{23}(v) R_{13}(u v) R_{12}(u) .
$$

To bring a quantum mechanical system into Lax form one has to arrange quantum observables into a Lax matrix $L(u)$ (which is a rational function of $u$ ) and to find an appropriate R-matrix satisfying (4.23), (4.24). The first examples of quantum Lax operators were constructed by trial and error method; in combination with the Bethe Ansatz technique this has led to the explicit solution of important problems (Sklyanin, Takhtajan and Faddeev (1979), Faddeev and Takhtajan (1979), Faddeev (1980, 1984)).

The algebraic concept which brings order to the subject is that of quasitriangular Hopf algebra (Drinfeld (1987a)). Main examples of quasitriangular Hopf algebras arise as q-deformations of universal enveloping algebras associated with Manin triples. Remarkably, the general pattern represented by Theorems 2.3, 4.1 survives q-deformation. The standard way to describe quantum deformations of simple finite-dimensional or affine Lie algebras is by means of generators and relations generalizing the classical Chevalley-Serre relations (Drinfeld (1987a), Jimbo (1986)). We shall recall this definition below in section 4.2.2 in the finite-dimensional case, and in section 4.2 .3 for the quantized universal enveloping algebra of the loop algebra $L\left(\mathfrak{s l}_{2}\right)$. A 
dual approach, due to Faddeev, Reshetikhin and Takhtajan (1989), is to construct quantum universal enveloping algebras as deformations of coordinate rings on Lie groups (regarded as linear algebraic groups). Of course, construction of a quantum deformation of the Poisson algebra $\mathcal{F}(G)$ was one of the first results of the quantum group theory and is, in fact, a direct generalization of the Baxter commutation relations $R T^{1} T^{2}=T^{2} T^{1} R$. A nontrivial fact, first observed by Faddeev, Reshetikhin and Takhtajan, is that the dual Hopf algebra (usually described as a q-deformation of the universal enveloping algebra) may also be regarded as a deformation of a Poisson algebra of functions on the dual group. More generally, the FRT construction is related to the quantum duality principle, which we shall now briefly discuss.

Let $\left(\mathfrak{g}, \mathfrak{g}^{*}\right)$ be a factorizable Lie bialgebra, $G, G^{*}$ the corresponding dual Poisson groups, $\mathcal{F}(G), \mathcal{F}\left(G^{*}\right)$ the associated Poisson-Hopf algebras of functions on $G, G^{*}$, and $\mathcal{F}_{q}(G), \mathcal{F}_{q}\left(G^{*}\right)$ their quantum deformations. (For simplicity we choose the deformation parameter $q$ to be the same for both algebras.) The quantum duality principle asserts that these algebras are dual to each other as Hopf algebras. More precisely, there exists a nondegenerate bilinear pairing

$$
\mathcal{F}_{q}(G) \otimes \mathcal{F}_{q}\left(G^{*}\right) \rightarrow \mathbb{C}[[q]]
$$

which sets the algebras $\mathcal{F}_{q}(G), \mathcal{F}_{q}\left(G^{*}\right)$ into duality as Hopf algebras. Hence, in particular, we have, up to an appropriate completion,

$$
\mathcal{F}_{q}\left(G^{*}\right) \simeq \mathcal{U}_{q}(\mathfrak{g}) .
$$

In the dual way, we have also

$$
\mathcal{F}_{q}(G) \simeq \mathcal{U}_{q}\left(\mathfrak{g}^{*}\right)
$$

For factorizable Lie bialgebras the quantum deformations $\mathcal{F}_{q}(G), \mathcal{F}_{q}\left(G^{*}\right)$ may easily be constructed once we know the corresponding quantum R-matrices. An equivalence of this formulation to the definition of Drinfeld and Jimbo is not immediate and requires the full theory of universal R-matrices. Namely, starting with Drinfeld's definition of a quasitriangular Hopf algebra we may construct 'quantum Lax operators' whose matrix coefficients generate the quantized algebras of functions $\mathcal{F}_{q}(G), \mathcal{F}_{q}\left(G^{*}\right)$. Using explicit formulae for universal R-matrices one can, in principle, express these generators in terms of the Drinfeld-Jimbo generators. In the context of integrable models the FRT formulation has several important advantages: it allows to state the quantum counterpart of the main commutativity theorem as well as a transparent correspondence between classical and quantum integrable systems. The analogue of the FRT realization in the affine case is nontrivial, the key point being the correct treatment of the central element which corresponds to the central extension; it was described by Reshetikhin and Semenov-Tian-Shansky (1990).

4.2.1. Factorizable Hopf algebras and the Faddeev-Reshetikhin-Takhtajan realization of quantized universal enveloping algebras.

Definition 4.2 Let $A$ be a Hopf algebra with coproduct $\Delta$ and antipode $S$; let $\Delta^{\prime}$ be the opposite coproduct in $A ; A$ is called quasitriangular if

$$
\Delta^{\prime}(x)=\mathcal{R} \Delta(x) \mathcal{R}^{-1}
$$


for all $x \in A$ and for some distinguished invertible element $\mathcal{R} \in A \otimes A$ (the universal R-matrix) and, moreover,

$$
(\Delta \otimes i d) \mathcal{R}=\mathcal{R}_{13} \mathcal{R}_{23},(i d \otimes \Delta) \mathcal{R}=\mathcal{R}_{13} \mathcal{R}_{12} .
$$

Identities (4.26) imply that $\mathcal{R}$ satisfies the Yang-Baxter identity

$$
\mathcal{R}_{12} \mathcal{R}_{13} \mathcal{R}_{23}=\mathcal{R}_{23} \mathcal{R}_{13} \mathcal{R}_{12} .
$$

(We use standard tensor notation to denote different copies of the spaces concerned.)

Let $A^{0}$ be the dual Hopf algebra equipped with the opposite coproduct (in other words, its coproduct is dual to the opposite product in $A$ ). Put $\mathcal{R}^{+}=\mathcal{R}, \mathcal{R}^{-}=\sigma\left(\mathcal{R}^{-1}\right.$ ) (here $\sigma$ is the permutation map in $A \otimes A, \sigma(x \otimes y)=y \otimes x)$ and define the mappings $R_{ \pm}: A^{0} \rightarrow A$ : $f \mapsto\left\langle f \otimes i d, \mathcal{R}^{ \pm}\right\rangle$; axioms (4.26) imply that $R_{ \pm}$are Hopf algebra homomorphisms. Define the action $A^{0} \otimes A \rightarrow A$ by

$$
f \circ x=\sum_{i} R^{+}\left(f_{i}^{(1)}\right) x S\left(R^{-}\left(f_{i}^{(2)}\right), \text { where } \Delta^{0} f=\sum_{i} f_{i}^{(1)} \otimes f_{i}^{(2)} .\right.
$$

Definition 4.3 $A$ is called factorizable if $A$ is a free $A^{0}$-module generated by $1 \in A$.

(Let us denote the corresponding linear isomorphism $A^{0} \rightarrow A$ by $F$ for future reference.)

There are several important examples of factorizable Hopf algebras:

- Let $A$ be an arbitrary Hopf algebra; then its Drinfeld double $D(A)$ is factorizable (Reshetikhin and Semenov-Tian-Shansky (1991)).

- Let $\mathfrak{g}$ be a finite-dimensional semisimple Lie algebra; then $A=\mathcal{U}_{q}(\mathfrak{g})$ is factorizable.

- Let $\hat{\mathfrak{g}}$ be an affine Lie algebra; then $A=\mathcal{U}_{q}(\hat{\mathfrak{g}})$ is factorizable.

(Observe that the last two cases are actually special cases of the first one; up to now, the double remains the principal (if not the only) source of factorizable Hopf algebras.)

Let $\mathfrak{g}$ be a simple Lie algebra. Let $A=\mathcal{U}_{q}(\mathfrak{g})$ be the corresponding quantized enveloping algebra, $A^{0}$ be the dual of $A$ with the opposite coproduct. Let $\mathcal{R}$ be the universal R-matrix of $A$. Let $V, W$ be finite-dimensional irreducible representations of $U_{q}(\mathfrak{g})$. Let $\mathcal{L} \in A \otimes A^{0}$ be the canonical element. Put

$$
L_{V}=\left(\rho_{V} \otimes i d\right) \mathcal{L}, R_{ \pm}^{V W}=\left(\rho_{V} \otimes \rho_{W}\right) \mathcal{R}_{ \pm} .
$$

We may call $L_{V} \in E n d V \otimes A^{0}$ the universal quantum Lax operator (with auxiliary space $V$ ). Property (4.25) immediately implies that

$$
L_{W}^{2} L_{V}^{1}=R_{+}^{V W} L_{V}^{1} L_{W}^{2}\left(R_{+}^{V W}\right)^{-1} .
$$

Proposition 4.18 The associative algebra $\mathcal{F}_{q}(G)$ generated by the matrix coefficients of $L_{V}$ satisfying the commutation relations (4.30) and with the matrix coproduct

$$
\Delta L_{V}=L_{V} \dot{\otimes} L_{V}
$$

is a quantization of the Poisson-Hopf algebra $\mathcal{F}(G)$ with the Poisson bracket (3.6), (3.8). 
The dual algebra $\mathcal{F}_{q}\left(G^{*}\right)$ is described in the following way. Let again $\mathcal{L} \in A \otimes A^{0}$ be the canonical element. Put

$$
\begin{gathered}
T_{V}{ }^{ \pm}=\left(\rho_{V} \otimes R^{ \pm}\right) \mathcal{L} \in E n d V \otimes A, \\
T_{V}=T_{V}{ }^{+}(i d \otimes S) T_{V}{ }^{-} .
\end{gathered}
$$

Proposition 4.19 (i) The matrix coefficients of $T_{V}{ }^{ \pm}$satisfy the following commutation relations:

$$
\begin{aligned}
& T_{W}^{ \pm 2} T_{V}^{ \pm 1}=R^{V W} T_{V}^{ \pm 1} T_{W}^{ \pm 2}\left(R^{V W}\right)^{-1}, \\
& T_{W}^{-2} T_{V}^{+1}=R^{V W} T_{V}^{+1} T_{W}^{-2}\left(R^{V W}\right)^{-1} . j
\end{aligned}
$$

(ii) The associative algebra $\mathcal{F}_{q}\left(G^{*}\right)$ generated by the matrix coefficients of $T^{ \pm V}$ satisfying the commutation relations (4.32) and with the matrix coproduct

$$
\Delta T^{ \pm V}=T^{ \pm V} \dot{\otimes} T^{ \pm V}
$$

is a quantization of the Poisson-Hopf algebra $\mathcal{F}\left(G^{*}\right)$ with Poisson bracket (3.9), (3.12).

(iii) $T_{V}=(i d \otimes F) L_{V}$. The matrix coefficients of $T_{V}$ satisfy the following commutation relations:

$$
\left(R_{+}^{V W}\right)^{-1} T_{W}^{2} R_{+}^{V W} T_{V}^{1}=T_{V}^{1}\left(R_{-}^{V W}\right)^{-1} T_{W}^{2} R_{-}^{V W} .
$$

(iv) The associative algebra generated by the matrix coefficients of $T_{V}$ satisfying the commutation relations (4.32) is a quantization of the Poisson-Hopf algebra $\mathcal{F}\left(G^{*}\right)$ with Poisson bracket (3.12).

(v) The pairing

$$
\left\langle T_{V}^{ \pm 1}, L_{W}^{2}\right\rangle=R_{+}^{V W}
$$

sets the algebras $\mathcal{F}_{q}(G), \mathcal{F}_{q}\left(G^{*}\right)$ into duality as Hopf algebras.

Formulae $(4.30,4.32,4.33)$ are the exact quantum analogues of the Poisson bracket relations $(3.8,3.9,3.12)$, respectively.

4.2.2. Quantum commutativity theorem and quantum Casimirs. In complete analogy with the linear case the appropriate algebra of observables which is associated with quantum Lax equations is not the quasitriangular Hopf algebra $A$ but rather its dual $A^{0}$; the Hamiltonians arise from the Casimir elements of $A$. We may summarize this picture in the following heuristic correspondence principle.

\begin{tabular}{|c|c|}
\hline Quadratic Classical Case & Quadratic Quantum case \\
\hline $\mathcal{F}(G)$ & $A^{0}=\mathcal{U}_{q}\left(\mathfrak{g}^{*}\right) \simeq \mathcal{F}_{q}(G)$ \\
\hline $\mathcal{F}\left(G^{*}\right)$ & $A=\mathcal{U}_{q}(\mathfrak{g}) \simeq \mathcal{F}_{q}\left(G^{*}\right)$ \\
\hline Classical r-matrices & Quantum $R$-matrices \\
\hline Casimir functions in $\mathcal{F}\left(G^{*}\right)$ & Casimir operators $\mathcal{Z} \subset \mathcal{U}_{q}(\mathfrak{g})$ \\
\hline Symplectic leaves in $G$ & Irreducible representations of $A^{0}$ \\
\hline Symplectic leaves in $G^{*}$ & Irreducible representations of $A$ \\
\hline
\end{tabular}

According to this correspondence principle, in order to get a 'quantum Lax system' associated with a given factorizable Hopf algebra $A$ we may proceed as follows: 
- Choose a representation $(\pi, \mathfrak{V})$ of $A^{0}$.

- Choose a Casimir element $\zeta \in \mathcal{Z}(A)$ and compute its inverse image $F^{-1}(\zeta) \in A^{0}$ with respect to the factorization map $F: A^{0} \rightarrow A$.

- Put $\mathcal{H}_{\zeta}=\pi\left(F^{-1}(\zeta)\right)$.

Moreover, we expect that the eigenvectors of the quantum Hamiltonian $\mathcal{H}_{\zeta}$ can be expressed as matrix coefficients of appropriate representations of $A$.

In order to describe quantum Casimirs explicitly we have to take into account the effect of twisting. We shall start with their description in the finite-dimensional case. The results stated below are the exact quantum counterparts of those described in section 3.2.2.

Let $\mathfrak{g}$ be a finite-dimensional simple Lie algebra, $A=\mathcal{U}_{q}(\mathfrak{g})$ the quantized universal enveloping algebra of $\mathfrak{g}$. We shall recall its standard definition in terms of the Chevalley generators (Drinfeld (1987a), Jimbo (1986)). Let $P$ be the set of simple roots of $\mathfrak{g}$. For $\alpha_{i} \in P$ we set $q_{i}=q^{\left\langle\alpha_{i}, \alpha_{i}\right\rangle}$. We denote the Cartan matrix of $\mathfrak{g}$. by $A_{i j}$

Definition $4.4 \mathcal{U}_{q}(\mathfrak{g})$ is a free associative algebra with the generators $\left\{k_{i}, k_{i}^{-1}, e_{i}, f_{i}\right\}_{i \in P}$ and relations

$$
\begin{array}{r}
k_{i} \cdot k_{i}^{-1}=k_{i}^{-1} k_{i}=1,\left[k_{i}, k_{j}\right]=0, \\
{\left[a_{i}, e_{j}\right]=q_{i}^{A_{i j}} e_{j},\left[a_{i}, f_{j}\right]=q_{i}^{-A_{i j}} e_{j},} \\
{\left[e_{i}, f_{j}\right]=\delta_{i j} \frac{\left(k_{i}^{2}-k_{i}^{-2}\right)}{q_{i}^{2}-q_{i}^{-2}}}
\end{array}
$$

and the following q-Serre relations:

$$
\begin{aligned}
& \sum_{n=0}^{1-A_{i j}}(-1)^{n}\left[\begin{array}{c}
1-A_{i j} \\
n
\end{array}\right]_{q_{i}^{2}} e_{i}^{1-A_{i j}-n} e_{j} e_{i}^{n}=0, \\
& \sum_{n=0}^{1-A_{i j}}(-1)^{n}\left[\begin{array}{c}
1-A_{i j} \\
n
\end{array}\right]_{q_{i}^{2}} f_{i}^{1-A_{i j}-n} f_{j} f_{i}^{n}=0 .
\end{aligned}
$$

(Here $\left[\begin{array}{c}m \\ n\end{array}\right]_{q}$ are the q-binomial coefficients.)

Theorem 4.20 (Drinfeld $(1987 a)) \mathcal{U}_{q}(\mathfrak{g})$ is a quasitriangular Hopf algebra.

As usual, we denote by $\mathcal{R} \in \mathcal{U}_{q}(\mathfrak{g}) \otimes \mathcal{U}_{q}(\mathfrak{g})$ its universal R-matrix and put $R_{V W}=\left(\rho_{V \otimes} \rho_{W}\right) \mathcal{R}$

We recall the following well known corollary of definition 4.4. Let $H$ be the 'Cartan subgroup' generated by the elements $k_{i}, i \in P, \mathbb{C}[H]$ its group algebra. We denote by $\mathcal{U}_{q}\left(\mathfrak{n}_{ \pm}\right)$, $\mathcal{U}_{q}\left(\mathfrak{b}_{ \pm}\right) \subset \mathcal{U}_{q}(\mathfrak{g})$ the subalgebras generated by $e_{i}, f_{i}$ (respectively, by $k_{i}, e_{i}$ and by $\left.k_{i}, f_{i}\right)$.

Proposition 4.21 (i) $\mathbb{C}[H], \mathcal{U}_{q}\left(\mathfrak{n}_{ \pm}\right), \mathcal{U}_{q}\left(\mathfrak{b}_{ \pm}\right)$are Hopf subalgebras in $\mathcal{U}_{q}(\mathfrak{g})$. (ii) $\mathcal{U}_{q}\left(\mathfrak{n}_{ \pm}\right)$is a two-sided Hopf ideal in $\mathcal{U}_{q}\left(\mathfrak{b}_{ \pm}\right)$and $\mathcal{U}_{q}\left(\mathfrak{b}_{ \pm}\right) / \mathcal{U}_{q}\left(\mathfrak{n}_{ \pm}\right) \simeq \mathbb{C}[H]$. 
Let $\pi_{ \pm}: \mathcal{U}_{q}\left(\mathfrak{b}_{ \pm}\right) \rightarrow \mathbb{C}[H]$ be the canonical projection. Consider the embedding

$$
i: \mathbb{C}[H] \rightarrow \mathbb{C}[H] \otimes \mathbb{C}[H]: a \longmapsto(i d \otimes S) \Delta a .
$$

(Here $\Delta$ is the coproduct in commutative and cocommutative Hopf algebra $\mathbb{C}[H]$ and $S$ is its antipode.)

Proposition 4.22 The dual of $\mathcal{U}_{q}(\mathfrak{g})$ may be identified with the subalgebra

$$
A^{0}=\left\{x \in \mathcal{U}_{q}\left(\mathfrak{b}_{+}\right) \otimes \mathcal{U}_{q}\left(\mathfrak{b}_{-}\right) ; \pi_{+} \otimes \pi_{-}(x) \in i(\mathbb{C}[H])\right\}
$$

Lemma 4.23 For any $h \in H, h \otimes h \mathcal{R}=\mathcal{R} h \otimes h$.

The algebra $A$ admits a (trivial) family of deformations $A_{h}=\mathcal{U}_{q}(\mathfrak{g})_{h}, h \in H$, defined by the following prescription:

$$
e_{i} \longmapsto h e_{i}, f_{i} \longmapsto h f_{i}, k_{i} \longmapsto h k_{i} .
$$

It is instructive to look at the commutation relations of $A_{h}$ in the FRT realization. Let $V, W \in$ $\operatorname{Rep} A$; we define the 'generating matrices' $T_{V}^{ \pm} \in A \otimes E n d V, T_{W}^{ \pm} \in A \otimes E n d W$, as in (4.31).

Proposition 4.24 (i) The deformation (4.34) transforms $T_{V}^{ \pm}$into $\rho_{V}\left(h^{\mp 1}\right) T_{V}^{ \pm}$; the commutation relations in the deformed algebra $A_{h}$ amount to

$$
\begin{aligned}
& T_{W}^{ \pm 2} T_{V}^{ \pm 1}=R^{V W} T_{V}^{ \pm 1} T_{V}^{ \pm 2}\left(R^{V W}\right)^{-1}, \\
& T_{W}^{-2} T_{V}^{+1}=R_{h}^{V W} T_{V}^{+1} T_{W}^{-2}\left(R^{V W}\right)^{-1},
\end{aligned}
$$

where $R_{h}^{V W}=\rho_{V} \otimes \rho_{W}\left(\mathcal{R}_{h}\right)$ and $\mathcal{R}_{h}=h \otimes h^{-1} \mathcal{R} h^{-1} \otimes h$.

(ii) Put $T_{h}=\rho_{V}\left(h^{2}\right) T_{V}^{+}\left(T_{V}^{-}\right)^{-1}$; the 'operator-valued matrix' $T_{h}$ satisfies the following commutation relations

$$
\left(R_{+}^{V W}\right)^{-1} T_{W}^{2} R_{+}^{V W} T_{V}^{1}=T_{V}^{1}\left(R_{-}^{V W}\right)^{-1} T_{W}^{2} R_{-}^{V W} .
$$

Let $L_{V} \in A^{*} \otimes E n d V$ be the 'universal Lax operator' introduced in (4.29). Fix $h \in H$ and put

$$
l_{V}^{h}=\operatorname{tr}_{V} \rho_{V}\left(h^{2}\right) L^{V} .
$$

Elements $l_{V}^{h} \in A^{0}$ are usually called (twisted) transfer matrices. The following theorem is one of the key results of QISM (as applied to the finite-dimensional case).

Theorem 4.25 For any representations $V, W$ and for any $h \in H$,

$$
l_{V}^{h} l_{W}^{h}=l_{W}^{h} l_{V}^{h}
$$


The mutual commutativity of transfer matrices is a direct corollary of the commutation relations (4.30); twisting by elements $h \in H$ is compatible with these relations, since

$$
(h \otimes h) \mathcal{R}=\mathcal{R}(h \otimes h) .
$$

We want to establish a relation between the transfer matrices and the Casimirs of $\mathcal{U}_{q}(\mathfrak{g})_{h^{\prime}}$ (where $h^{\prime} \in H$ may be different from $h$ ). To account for twisting we must slightly modify the factorization map.

Let $H \times A \rightarrow A$ be the natural action of $H$ on $A$ by right translations, and let $H \times A^{0} \rightarrow A^{0}$ be the contragredient action. The algebra $A=\mathcal{U}_{q}(\mathfrak{g})$ is factorizable; let $F: A^{0} \rightarrow A$ be the isomorphism induced by the action (4.28); for $h \in H$ put $F^{h}=F \circ h$. Let us compute the image of the universal Lax operator $L_{V} \in E n d V \otimes A^{0}$ under the 'factorization mapping' $\left(i d \otimes F^{h}\right)$; it is easy to see that

$$
\left(i d \otimes F^{h}\right)\left(L_{V}\right):=T_{V}^{h \pm}=\left(i d \otimes h^{ \pm 1}\right) T_{V}^{1 \pm}
$$

Put

$$
t_{V}^{h}=t r_{V} T_{V}^{h+}\left(T_{V}^{h-}\right)^{-1}=t r_{V} \rho_{V}(h)^{2} T_{V}^{1}
$$

clearly, we have $t_{V}^{h}=F\left(l_{V}^{h}\right)$.

Theorem 4.26 (i) (Faddeev, Reshetikhin and Takhtajan (1989)) Suppose that $h=q^{-\rho}$, where $2 \rho \in \mathfrak{h}$ is the sum of positive roots of $\mathfrak{g}$. Then all coefficients of $t_{V}^{h}$ are central in $A=\mathcal{U}_{q}(\mathfrak{g})$. (ii) The center of $A$ is generated by $\left\{t_{V}^{h}\right\}$ (with $V$ ranging over all irreducible finite-dimensional representations of $\left.\mathcal{U}_{q}(\mathfrak{g})\right)$.(iii) For any $s \in H$ we have $l_{V}^{s}=F^{s h^{-1}}\left(t_{V}^{h}\right)$.

Theorem 4.26 is an exact analogue of theorem 4.1 for finite-dimensional factorizable quantum groups. Its use is twofold: first, it provides us with commuting quantum Hamiltonians; second, the eigenfunctions of these Hamiltonians may be constructed as appropriate matrix coefficients ('correlation functions') of irreducible representations of $\mathcal{U}_{q}(\mathfrak{g})$. Again, to apply the theorem to more interesting and realistic examples we must generalize it to the affine case.

4.2.3. Quantized affine Lie algebras. Two important classes of infinite-dimensional quasitriangular Hopf algebras are quantum affine Lie algebras and the full Yangians (that is, the doubles of the Yangians defined in (Drinfeld (1987a))). For concreteness I shall consider only the first class. To avoid technical difficulties we shall consider the simplest nontrivial case, that of the affine $\mathfrak{s l}_{2}$.

The standard definition of $\mathcal{U}_{q} \widehat{\left(\mathfrak{s l}_{2}\right)}$ is by means of generators and relations:

Definition $4.5 \mathcal{U}_{q} \widehat{\left(\mathfrak{s l}_{2}\right)}$ is a free associative algebra over $\mathbb{C}\left[q, q^{-1}\right]$ with generators $e_{i}, f_{i}, K_{i}$, $K_{i}^{-1}, i=0,1$, which satisfy the following relations:

$$
\begin{aligned}
K_{i} K_{j} & =K_{j} K_{i}, \\
K_{i} e_{j} & =q^{A_{i j}} e_{j} K_{i}, K_{i} f_{j}=q^{-A_{i j}} f_{j} K_{i}, \\
{\left[e_{i}, f_{j}\right] } & =\delta_{i j}\left(q-q^{-1}\right)\left(K_{i}-K_{i}^{-1}\right),
\end{aligned}
$$


where $A_{i j}$ is the Cartan matrix of the affine $\mathfrak{s l}_{2}, A_{00}=A_{11}=-A_{01}=-A_{10}=2$. In addition to (4.40) the following q-Serre relations are imposed:

$$
\begin{aligned}
e_{i}^{3} e_{j}-\left(1+q+q^{-1}\right)\left(e_{i}^{2} e_{j} e_{i}-e_{i} e_{j} e_{i}^{2}\right)-e_{j} e_{i}^{3}=0, \\
f_{i}^{3} f_{j}-\left(1+q+q^{-1}\right)\left(f_{i}^{2} f_{j} f_{i}-f_{i} f_{j} f_{i}^{2}\right)-f_{j} f_{i}^{3}=0 .
\end{aligned}
$$

The Hopf structure in $\mathcal{U}_{q} \widehat{\left(\mathfrak{s l}_{2}\right)}$ is defined by

$$
\begin{aligned}
\Delta K_{i} & =K_{i} \otimes K_{i}, \\
\Delta e_{i} & =e_{i} \otimes 1+K_{i} \otimes e_{i}, \\
\Delta f_{i} & =f_{i} \otimes 1+K_{i} \otimes e_{i} .
\end{aligned}
$$

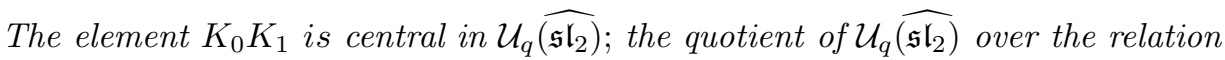

$$
K_{0} K_{1}=q^{k}, k \in \mathbb{Z},
$$

is called the level $k$ quantized universal enveloping algebra $\mathcal{U}_{q} \widehat{\left(\mathfrak{s l}_{2}\right)_{k}}$.

An alternative realization of $\mathcal{U}_{q} \widehat{\left(\mathfrak{s l}_{2}\right)_{k}}$ is based on the explicit use of the quantum R-matrix; in agreement with the quantum duality principle, this realization may be regarded as an explicit quantization of the Poisson algebra of functions $\mathcal{F}_{q}\left(G^{*}\right)$, where $G^{*}$ is the Poisson dual of the loop group $L\left(S L_{2}\right)$. The relevant Poisson structure on $G^{*}$ has to be twisted in so as to take into account the central charge $k$; moreover, in complete analogy with the non-deformed case, the center of $\mathcal{U}_{q} \widehat{\left(\mathfrak{s l}_{2}\right)_{k}}$ is nontrivial only for the critical value of the central charge $k_{\text {crtit }}=-2$. (The critical value $k=-2$ is a specialization, for $\mathfrak{g}=\mathfrak{s l}_{2}$, of the general formula $k=-h^{\vee}$; thus the value of $k_{\text {crit }}$ is not affected by q-deformation.)

Introduce the R-matrix

$$
R(z)=\left(\begin{array}{cccc}
1 & 0 & 0 & 0 \\
0 & \frac{1-z}{q-z q^{-1}} & \frac{z\left(q-q^{-1}\right)}{q-z q^{-1}} & 0 \\
0 & \frac{q-q^{-1}}{q-z q^{-1}} & \frac{1-z}{q-z q^{-1}} & 0 \\
0 & 0 & 0 & 1
\end{array}\right)
$$

Let $\mathcal{U}_{q}^{\prime}{\widehat{\left(\mathfrak{g l}_{2}\right)_{k}}}_{k}$ be an associative algebra with generators $l_{i j}^{ \pm}[n], i, j=1,2, n \in \pm \mathbb{Z}+$. In order to describe commutation relations in $\mathcal{U}_{q}^{\prime}{\widehat{\left(\mathfrak{g l} l_{2}\right)}}_{k}$ we introduce the generating series $L^{ \pm}(z)=\left\|l_{i j}^{ \pm}(z)\right\|$,

$$
l_{i j}^{ \pm}(z)=\sum_{n=0}^{\infty} l_{i j}^{ \pm}[ \pm n] z^{ \pm n}
$$


we assume, moreover, that $l_{i j}^{ \pm}[0]$ are upper (lower) triangular: $l_{i j}^{+}[0]=l_{j i}^{-}[0]=0$ for $i<j$. The defining relations in $\mathcal{U}_{q}^{\prime} \widehat{\left(\mathfrak{g l}_{2}\right)_{k}}$ are

$$
\begin{aligned}
l_{i i}^{+}[0] l_{i i}^{-}[0] & =l_{i i}^{-}[0] l_{i i}^{+}[0]=1, i=1,2, \\
R\left(\frac{z}{w}\right) L_{1}^{ \pm}(z) L_{2}^{ \pm}(w) & =L_{2}^{ \pm}(w) L_{1}^{ \pm}(z) R\left(\frac{z}{w}\right), \\
R\left(\frac{z}{w} q^{-k}\right) L_{1}^{+}(z) L_{2}^{-}(w) & =L_{2}^{-}(w) L_{1}^{+}(z) R\left(\frac{z}{w} q^{k}\right)
\end{aligned}
$$

(we use again the standard tensor notation). Relations (4.45) are understood as relations between formal power series in $\frac{z}{w}$. Observe the obvious parallels between (4.45) and (4.35): the shift in the argument of $R$ reflects the effects of the central extension; in the finite-dimensional case this extension is of course trivial and the shift may be eliminated by a change of variables. The commutation relations (4.45) are the exact quantum analogue of the Poisson bracket relations (3.26) with $p=q^{k}$.

Lemma 4.27 The coefficients of the formal power series

$$
q \operatorname{det}\left(L^{ \pm}(z)\right):=l_{11}^{ \pm}\left(z q^{2}\right)\left(l_{22}^{ \pm}(z)-l_{21}^{ \pm}(z) l_{11}^{ \pm}(z)^{-1} l_{12}^{ \pm}(z)\right)
$$

are central in $\mathcal{U}_{q}^{\prime}\left(\widehat{\mathfrak{g l}_{2}}\right)_{k}$.

Theorem 4.28 The quotient of $\mathcal{U}_{q}^{\prime}\left(\widehat{\mathfrak{g l}_{2}}\right)_{k}$ over the relations $q \operatorname{det}\left(L^{ \pm}(z)\right)=1$ is isomorphic to $\mathcal{U}_{q} \widehat{\left(\mathfrak{s l}_{2}\right)_{k}}$.

The accurate proof of this theorem (and of its generalization to arbitrary quantized affine Lie algebras) is far from trivial (see Ding and Frenkel (1994)); it relies on a still another realization of quantized affine Lie algebras, the so-called Drinfeld's new realization (Drinfeld (1987b)); the important point for us is that the realization described in theorem 4.28 is adapted to the explicit description of the Casimir elements.

Let us put

$$
L(z)=L^{+}\left(q^{-k / 2} z\right) L^{-}\left(z q^{k / 2}\right)^{-1}
$$

(this is the so-called full quantum current)

Theorem 4.29 (Reshetikhin and Semenov-Tian-Shansky (1990)) (i) The quantum current (4.46) satisfies the commutation relations

$$
\begin{gathered}
R\left(\frac{z}{w}\right) L_{1}(z) R^{-1}\left(\frac{q^{-k / 2} z}{w}\right) L_{2}(w)= \\
L_{2}(w) R\left(\frac{q^{k / 2} z}{w}\right) L_{1}(z) R\left(\frac{z}{w}\right)^{-1} .
\end{gathered}
$$

(ii) Suppose that $k=-2$; then the coefficients of the formal series

$$
t(z)=\operatorname{tr} q^{2 \rho} L(z)
$$

are central elements in the algebra $\mathcal{U}_{q} \widehat{\left(\mathfrak{s l}_{2}\right)_{k}}$. 
As usual, $\rho$ stands for half the sum of positive roots. In the present setting we have simply $q^{2 \rho}=\left(\begin{array}{cc}q & 0 \\ 0 & q^{-1}\end{array}\right)$. The critical value $k=-2$ is a special case of the general formula $k=-h^{\vee}$ (the dual Coxeter number); thus the critical value remains the same as in the non-deformed case.

The definition of the quantum current and formulae $(4.47,4.48)$ are in fact quite general and apply to arbitrary quantized loop algebras. There are all reasons to expect that the center of the quotient algebra $A_{-h^{\vee}}=A /\left(c+h^{\vee}\right)$ for the critical value of the central charge is generated by (4.48) (with $V$ ranging over all irreducible finite-dimensional representations of $\mathcal{U}_{q}(\widehat{\mathfrak{g}})$ ) (cf. (Ding and Etingof (1994), Frenkel (1995))).There are two main subtle points:

1. Our construction involved the 2-dimensional 'evaluation representation' $\mathcal{U}_{q}\left(\mathfrak{s l}_{2}\right) \rightarrow$ $E n d \mathbb{C}((z))$. In the general case we must describe finite-dimensional representations $V(z)$ of $\mathcal{U}_{q}(\mathfrak{g})$ with spectral parameter; this requires additional technical efforts.

2. In the $\mathfrak{s l}_{2}$ case it was possible to use the generating series (4.44) as an exact alternative to the Drinfeld-Jimbo definition; in the general case the analogues of relations (4.45) are still valid but there may be others; anyway, the generating series $L_{V(z)}^{ \pm}$may be defined as the image of the canonical element:

$$
L_{V(z)}^{ \pm}=\left(i d \otimes \rho_{V(z)}\right) \circ\left(R_{ \pm} \otimes i d\right) L ;
$$

the definition of the quantum current and theorem 4.29 are still valid.

We may now use theorem 4.29 to relate the elements of the center to quantum Hamiltonians. Our main pattern remains the same. For concreteness let us again assume that $A=$ $\mathcal{U}_{q} \widehat{\left(\mathfrak{s l}_{2}\right)}$. Let us take its dual $A^{0}$ as the algebra of observables. Let $\mathcal{L} \in A \otimes A^{0}$ be the canonical element. For any finite-dimensional $\mathfrak{s l}_{2}$-module $V$ there is a natural representation of $A$ in $V((z))$ (evaluation representation) Let $W$ be another finite-dimensional $\mathfrak{s l}_{2}$-module. Put $L^{V}(z)=\left(\rho_{V(z)} M \otimes i d\right) \mathcal{L}, L^{W}(z)=\left(\rho_{W(z)} \otimes i d\right) \mathcal{L}, R^{V W}(z)=\left(\rho_{V(z)} \otimes \rho_{W(z)}\right) \mathcal{R}$. We may call $L^{V}(z) \in \operatorname{EndV}((z)) \otimes A^{0}$ the universal quantum Lax operator (with auxiliary space $V$ ). Fix a finite-dimensional representation $\pi$ of $A^{0}$; one can show that $(i d \otimes \pi) L_{V}(z)$ is rational in $z$. (Moreover, Tarasov (1985) showed how to use this dependence on $z$ to classify finite-dimensional representations of $A^{0}$.) Property (4.25) immediately implies that

$$
L_{2}^{W}(w) L_{1}^{V}(v)=R^{V W}\left(v w^{-1}\right) L_{1}^{V}(v) L_{2}^{W}(w)\left(R^{V W}\left(v w^{-1}\right)\right)^{-1} .
$$

Moreover, $R^{V W}(z)$ satisfies the Yang-Baxter identity (4.24).

Let $H$ be the Cartan subgroup of $G$. Fix $h \in H$ and put $l_{V}^{h}(v)=\operatorname{tr}_{V} \rho_{V}\left(h^{2}\right) L^{V}(v)$, then $l_{V}^{h}(v) l_{W}^{h}(w)=l_{W}^{h}(w) l_{V}^{h}(v)$. Elements $l_{V}^{h}(v)$ are called (twisted) transfer matrices.

It is convenient to extend $A$ by adjoining to it group-like elements which correspond to the extended Cartan subalgebra $\hat{\mathfrak{h}}=\mathfrak{h} \oplus \mathbb{C} d$ in $\hat{\mathfrak{g}}$. Let $\hat{H}=H \times \mathbb{C}^{*}$ be the corresponding "extended Cartan subgroup' in $A$ generated by elements $h=q^{\lambda}, \lambda \in \mathfrak{h}, t=q^{k d}$. Let $\hat{H} \times A \rightarrow A$ be 
its natural action on $A$ by right translations, $\hat{H} \times A^{0} \rightarrow A^{0}$ the contragredient action. The algebra $A=\mathcal{U}_{q}(\widehat{\mathfrak{g}})$ is factorizable; let $F: A^{0} \rightarrow A$ be the isomorphism induced by the action (4.28); for $h \in \hat{H}$ put $F^{h}=F \circ h$.

$$
\begin{gathered}
L_{V}^{h}(z)^{ \pm}=\left(i d \otimes R^{ \pm} \circ h\right) L_{V}(z) \in \operatorname{EndV}\left[\left[z^{ \pm 1}\right]\right] \otimes A \\
L_{V}^{h}(z)=L_{V}^{h}(z)^{+}(i d \otimes S) L_{V}^{h}(z)^{-}, h \in \hat{H}
\end{gathered}
$$

it is easy to see that

$$
L_{V}^{h}(z)^{ \pm}=\left(i d \otimes h^{ \pm 1}\right) L_{V}^{1}(z)^{ \pm}
$$

and hence

$$
T_{V}^{h}(z)=(i d \otimes h) T_{V}^{1}(z)(i d \otimes h) ;
$$

moreover, if $h=s^{-1} t$, where $s \in H$ and $t=q^{k d}$, we have also

$$
T_{V}^{h}(z)=\left(\rho_{V}(s) \otimes i d\right) T_{V}^{t}(z)\left(\rho_{V}(s) \otimes i d\right) .
$$

Put $t_{V}^{h}=\operatorname{tr}_{V} T_{V}^{h}(z)=\operatorname{tr}_{V} \rho_{V}(s)^{2} T_{V}^{t}(z)$; clearly, we have $t_{V}^{h}(z)=F^{t}\left(l_{V}^{s}(z)\right)$. Let $2 \rho$ be the sum of positive roots of $\mathfrak{g}=\mathfrak{s l}_{2}, h^{\vee}=2$ its dual Coxeter number (our notation again hints at the general case).

Theorem 4.30 (i) (Reshetikhin and Semenov-Tian-Shansky (1990)) Suppose that $h=$ $q^{-\rho} q^{-h^{\vee} d}$. Then all coefficients of $t_{V}^{h}(z)$ are central in $U$. (ii) For any $s \in H$ we have $l_{V}^{s}(z)=$ $F^{s h^{-1}}\left(t_{V}^{h}(z)\right)$.

Thus the duality between Hamiltonians and Casimir operators holds for quantum affine algebras as well. This allows to anticipate connections between the generalized Bethe Ansatz, representation theory of quantum affine algebras at the critical level and the q-KZ equation (Frenkel and Reshetikhin (1992), Smirnov (1992)). The results bearing on these connections are already abundant (Ding and Etingof (1994), Tarasov and Varchenko (1994), Frenkel (1995)), although they are still not in their final form.

As we have already observed in the classical context, the Hopf structure on $A^{0}$ is perfectly suited to the study of lattice systems. Let $\Delta^{(N)}: A^{0} \rightarrow \otimes^{N} A^{0}$ be the iterated coproduct map. $\otimes^{N} A^{0}$ may be interpreted as the algebra of observables associated with a multiparticle system. Put $\hat{t}_{V}^{h}(z)=\Delta^{(N)} t_{V}^{h}(z)$; Laurent coefficients of $\hat{t}_{V}^{h}(z)$ provide a commutative family of Hamiltonians in $\otimes^{N} A^{0}$. Let $i_{n}: A^{0} \rightarrow \otimes^{N} A^{0}$ be the natural embedding, $i_{n}: x \mapsto 1 \otimes \ldots \otimes x \otimes$ $\ldots \otimes 1$; put $L_{V}^{n}=\left(i d \otimes i_{n}\right) L_{V}$. Then

$$
\hat{t}_{V}^{h}(z)=\operatorname{tr}_{V}\left(\rho_{V}(h) \overleftarrow{\prod_{n}} L_{V}^{n}\right)
$$

Formula (4.51) has a natural interpretation in terms of lattice systems: $L_{V}^{n}$ may be regarded as 'local' Lax operators attached to the points of a periodic lattice $\Gamma=\mathbb{Z} / N \mathbb{Z}$; commuting Hamiltonians for the big system arise from the monodromy matrix $M_{V}=\overleftarrow{\prod} L_{V}^{n}$ associated with the lattice. Finally, the twist $h \in H$ defines a quasiperiodic boundary condition on the 
lattice. The study of the lattice system again breaks into two parts: (a) Find the joint spectrum of $\hat{l}_{V}(z)$. (b) Reconstruct the Heisenberg operators corresponding to 'local' observables and compute their correlation functions. This is the Quantum Inverse Problem (profound results on it are due to Smirnov (1992).)

\section{References}

Adler M. (1979). On a trace functional for formal pseudodifferential operators and the symplectic structure for the KdV type equations, Invent. Math. 50, 219-248.

Baxter R.J. (1982) Exactly Solved Models in Statistical Mechanics. Academic Press, London.

Belavin A.A., Drinfeld V.G. (1983). Triangle equations and simple Lie algebras, Sov. Sci. Rev., 4C, 93-165.

Babujian H.M., Flume R. (1993). Off-shell Bethe ansatz equations for Gaudin magnets and solutions of KZ equations, Preprint Bonn-HE-93-30.

Bogolyubov N.M., Izergin A.G., Korepin V.E. (1994). Correlation Functions of Integrable Systems and Quantum Inverse Scattering Method. Cambridge University Press, Cambridge.

Bayen F., Flato M., Fronsdal C., Lichnerowicz A., Sternheimer D. (1977). Deformation theory and quantization, I, II. Ann. Phys., 111, 61-151.

Cartier P. (1994). Some fundamental techniques in the theory of integrable systems, in: Lectures on integrable systems, O. Babelon, P. Cartier, Y. Kosmann-Schwarzbach, eds. World Scientific.

Ding J., Etingof P. (1994). Center of a quantum affine algebra at the critical level. Math. Res. Lett. 1, 469-480.

Ding J,. Frenkel I. (1993). Commun. Math. Phys.156,277-300.

Dixmier J. (1974). Algèbres enveloppantes. Cahiers Scientifiques, fasc. XXXVII. Gauthier-Villars, Paris.

Drinfeld V.G. (1983). Hamiltonian structures on Lie groups, Lie bialgebras and the geometric meaning of the classsical Yang-Baxter equation. Sov. Math. Dokl., 27, 68-71.

Drinfeld V.G. (1987). Quantum Groups. Proc. ICM-86 (Berkeley), vol. 1, p. 798. AMS.

Drinfeld V.G. (1987). Sov. Math. Dokl., 36, 212-216.

Faddeev L.D. (1980). Quantum Completely Integrable Systems in Quantum Field Theory. Sov. Sci. Rev.1C, 107-155.

Faddeev L.D. (1984). Integrable Models in (1+1)-dimensional Quantum Field Theory. Les Houches 1982. Elsevier Science Publ.

Faddeev L.D. (1995). Algebraic aspects of Bethe ansatz, Int. J. Mod. Phys. A10, 1845-1878, e-print archive: hep-th/9404013.

Faddeev L.D., Reshetikhin N.Yu., Takhtajan L.A. (1989). Quantization of Lie groups and Lie algebras. Leningrad Math. J., 1, 178-207.

Faddeev L.D., Takhtajan L.A. (1979). Quantum Inverse Scattering Method. Russian Math. Surveys, 34:5, 11-68.

Faddeev L.D., Takhtajan L.A. (1987). Hamiltonian Methods in the Theory of Solitons. Springer.

Faddeev L.D., Volkov A.Yu. (1993). Abelian current algebra and the Virasoro algebra on the lattice. Phys. Lett. B315, 311-318.

Feigin B, Frenkel E. (1992). Affine Kac-Moody algebras at the critical level and Gelfand-Dikij algebras, Int. J. Mod. Phys.A 7, Suppl. 1A, 197-215. 
Feigin B., Frenkel E., and Reshetikhin N. (1994). Gaudin model, Bethe ansatz and correlation functions at the critical level. Commun. Math.Phys, 166, 27-62.

Frenkel E. (1995). Affine algebras, Langlands duality and Bethe ansatz. Proc. International Congr. of Math. Physics, Paris 1994. Paris, International Press, pp.606-642.

Frenkel I.B., Reshetikhin N.Yu. (1992). Quantum affine algebras and holonomic difference equations. Commun. Math. Phys. 146, 1-60.

Gardner C.S., Greene J.M., Kruskal M.D., Miura R.M. (1967). Method for solving the KdV equation, Phys. Rev. Lett. 19, 1095-1097.

Gaudin M. (1983). La Fonction d'Onde de Bethe. Masson, Paris.

Goodman R., and Wallach N.R. (1982). Classical and quantum-mechanical systems of Toda lattice type. I. Commun. Math. Phys., 83, 355-386.

Goodman R., and Wallach N.R. (1984). Classical and quantum-mechanical systems of Toda lattice type.II. Commun. Math. Phys., 94, 177-217

Harnad J., Winternitz P. (1993). Classical and quantum integrable systems in $\tilde{\mathbf{g l}} \mathbf{l}(2)^{+*}$ and separation of variables. Preprint CRM 1921, Montreal 1993.

Jimbo M. (1986). A q-difference analogue of $U(\mathfrak{g})$ and the Yang-Baxter equation. Lett. Math. Phys. 10, 62-69.

Jurčo B. (1989). Classical Yang-Baxter equation and quantum integrable systems. J. Math. Phys. 30, 1289-1293.

Kazhdan D., Kostant B., Sternberg S. (1978). Hamiltonian group actions and dynamical systems of Calogero type. Commun. Pure Appl. Math. 31, 481-508.

Kirillov A.A. (1976). Elements of representation theory. Springer Verlag. Berlin a.o.

Kirillov A.N., Reshetikhin N.Yu. (1990). q-Weyl group and a multiplicative formula for the universal R-matrix, Commun. Math. Phys. 134, 421- 431

Kostant B. (1978). On Whittaker vectors and representation theory, Inventiones Math. 48, 101-184.

Kostant B. (1979). Quantization and Representation Theory, in: Representation Theory of Lie Groups, Proc. SRC/LMS Res. Symp., Oxford 1977. London Math. Soc. Lecture Notes Series, 34, 287-316, 1979.

Kulish P.P., Sklyanin E.K. (1981). Quantum Spectral Transform Method. In: Integrable Quantum Field Theories. Lecture Notes in Physics 151, 61-119, Springer.

Kuznetsov V. (1994). Equivalence of two graphical calculi. J.Phys. A25, 6005-6026.

Lax P.D. (1968). Integrals of nonlinear equations and solitary waves. Commun. Pure Appl. Math., 21, 467-490.

Olshanetsky M.A., Perelomov A.M. (1994). Integrable systems and finite-dimensional Lie algebras, in: Encyclopaedia of Math. Sciences, vol. 16. Springer-Verlag, Berlin a.o.

Reshetikhin N.Yu., Semenov-Tian-Shansky M.A. (1990). Central extensions of quantum current groups. Lett. Math. Phys. 19, 133-142 (1990).

Reshetikhin N.Yu., Semenov-Tian-Shansky M.A. (1991). Quantum R-matrices and factorization problems, in: Geometry and Physics, essays in honour of I.M.Gelfand, S.Gindikin and I.M.Singer, eds. North Holland, Amsterdam - London - New York, 1991, pp. 533-550.

Reshetikhin N., Varchenko A. (1993). Quasiclassical asymptotics of the KZ equations. Preprint.

Reyman A.G. (1996). Poisson structures related to quantum groups, in: International School of Physics "E. Fermi", Course 127, Varenna, 1994. Quantum Groups and their Applications in Physics, L. Castellani and J. Wess, eds. Amsterdam, 1996. 
Reyman A.G., Semenov-Tian-Shansky M.A. (1994). Group-theoretical methods in the theory of finitedimensional integrable systems, in: Encyclopaedia of Math. Sciences, vol. 16. Springer-Verlag, Berlin a.o.

Semenov-Tian-Shansky M.A. (1983). What is the classical r-matrix. Funct Anal. Appl. 17, 259-272.

Semenov-Tian-Shansky M.A. (1994a) Quantization of open Toda lattice, in: Encyclopaedia of Math. Sciences, vol. 16. Springer-Verlag, Berlin a.o. 1994.

Semenov-Tian-Shansky M.A. (1994b). Lectures on r-matrices, Poisson Lie groups and integrable systems, in: Lectures on integrable systems, O. Babelon, P. Cartier, Y. Kosmann-Schwarzbach, eds. World Scientific, 1994.

Semenov-Tian-Shansky M.A. (1994c). Poisson Lie groups, quantum duality principle and the quantum double. Contemporary Math., 175, 219-248.

Sklyanin E.K. (1979) On complete integrability of the Landau-Lifshitz equation. Preprint LOMI E-3-79, Leningrad, 1979.

Sklyanin E.K. (1992). Quantum Inverse Scattering Method. Selected Topics, in: Quantum Groups and Quantum Integrable Systems. Ge M.L., ed. World Scientific, Singapore, 1992.

Sklyanin E.K. (1995). Separation of variables. New trends, in: QFT, Integrable Models and beyond, T. Inami and R. Sasaki, eds. Progr. Theor. Phys. Suppl.118, 35-60.

Sklyanin E.K., Takhtajan L.A., Faddeev L.D. (1979). The quantum inverse scattering method. I. Theor. Math. Phys. 101, 194-220.

Smirnov F.A. (1992). Form Factors in Completely Integrable Models of Quantum Field Theory. Adv. Series in Math. Phys. 14. World Scientific, Singapore.

Tarasov V.O. (1985). Irreducible monodromy matrices for the R-matrix of the XXZ-model and lattice local quantum Hamiltonians. Theor. Math. Phys. 63, 440-454.

Tarasov V.O., Varchenko A. (1994). Jackson integral representations for solutions of the quantized KZ equation. St. Petersburg Math. J. 6, no 2.

Weinstein A. (1994). Deformation quantization. Seminaire Bourbaki, exposé n 789. 